\title{
How do caterpillars detect vibration? \\ Proleg sensory hairs as vibration receptors in Drepana arcuata (Drepanidae) and Trichoplusia ni (Noctuidae)
}

By

Conrado Denadai

A dissertation submitted to the Faculty of Graduate Studies and Research in partial fulfillment of the requirements for the degree of

Master of Science

in

Biology

\author{
Carleton University \\ Ottawa, Canada \\ (C) 2018 \\ Conrado Denadai
}




\begin{abstract}
It is known that substrate-borne vibrations are detected by insects from multiple taxa and life stages, including adults and larvae. Therefore, in this thesis I aimed to answer the question "How do caterpillars detect vibration?". For this purpose, I made neurophysiological experiments on proleg hairs of Drepana arcuata and Trichoplusia ni caterpillars. In these experiments, two types of mechanical stimuli were applied to the proleg hair: 1) sine vibrational signals with varying frequencies and 2) single push/pull stimuli. Amplitudes of stimuli ranged from sub- to supra-threshold and stimuli were applied in multiple directions to test for sensory directionality. With this, I showed that the sensory activity of the hair can be characterized as a rapid adapting response, that it has directional sensitivity and presents some phase-locking. In conclusion, the sensory hairs here evaluated can respond to vibration, which leaves them as potential candidates for substrate-borne vibration receptors in these caterpillars.
\end{abstract}




\section{Acknowledgements}

I will start by thanking a couple friends that were present in the lab several years ago, when I was still an undergrad. Thank you, Andrew Mikhail, for letting me learn from your neurophysiological experiments, and Amanda Lindeman, for the advice in my own experiments and ideas. Thank you, Dobai Andras, for teaching me how to use all the recording apparatus, how to manufacture electrodes and for giving me the Tucker-Davis files without which I could not have built my stimulation protocol. Thank you, Sheldon Maschmeyer, for helping me adapting the micro-manipulators to hold the Mini-shaker, something that had kept me busy for days until you helped me. Thank you, Dr. Jeff Dawson, for always keeping your door open, welcoming me to answer questions and fixing my broken electrode, the same that was later used to record the first neural response of this thesis. Thank you, Dr. John Lewis, for the clear feedback on my analyses, for helping me understanding my own experiment, for the suggestions that gave rise to several results in this thesis and for increasing my self-confidence when I needed it. Thank you, my friends from the Yack Lab: Melanie, Carrie, Mairelys, Andras, Amanda Dookie and Chanchal - who by the way still owes me a BeaverTail. You are always fun and supportive, and my only regret is not having spent more time with you. Thank you, Darlene Moss and Caitlyn McKenzie, for your assistance and enthusiasm, since the first day I met you. Thank you, Ed, because your smile has made me happy many times. And thank you Dr. Jayne Yack, who did not only give me the professional opportunities of taking my Master's in Canada, participate in several international conferences and publish papers, but also was my reason and motivation to come in this 
journey. I thank your patience in advising me, your promptness in reading through my long emails every time and your dedication in reviewing any of my pieces of work, even when time was short for me and busy for you. Thank you for the great moments, for having me in your home as your own family, for the conversations during and after work and for caring for me. It was all worthy!

The work in this thesis was funded to Dr. Jayne Yack and myself, by Carleton University, the Canada Foundation for Innovation (CFI), the Early Researcher Award (ERA), the Natural Sciences and Engineering Research Council (NSERC) and the Ontario Innovation Trust. 


\section{Table of Contents}

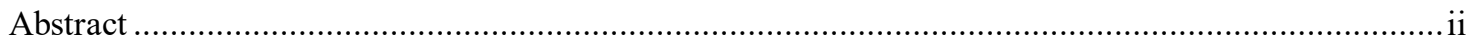

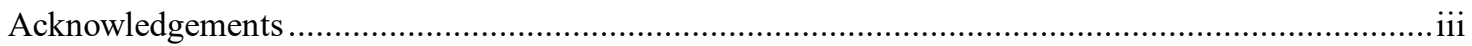

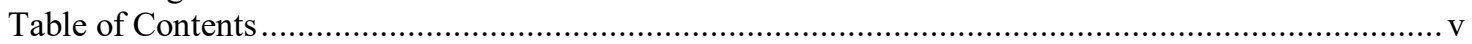

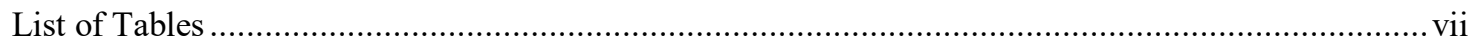

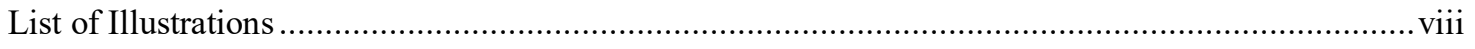

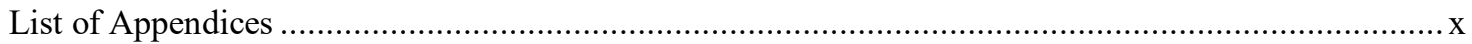

Chapter 1 General Introduction .............................................................................................1

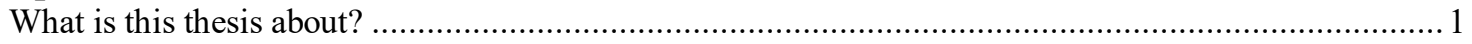

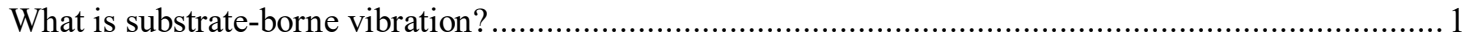

What physical parameters are important to characterize substrate-borne vibrations? ..........................5

What is the transduction mechanism of mechanical waves in the molecular level? .......................... 10

What cell types and sensilla are involved in mechano-transduction in insects?............................... 13

What physical parameters of mechanical waves do insects` sensors receive and transduce into

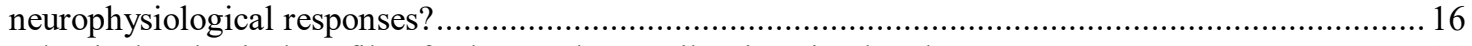

What is the physical profile of substrate-borne vibrations in plant leaves? ...................................20

After all, what is a substrate-borne vibration receptor? ........................................................ 23

Chapter 2 Vibration-sensitive hair on the proleg of Trichoplusia ni (Noctuidae): velocity

thresholds, directionality and intensity of response .....................................................27

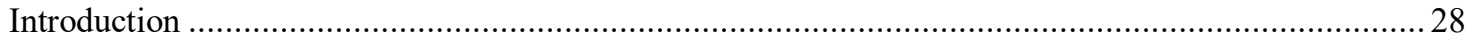

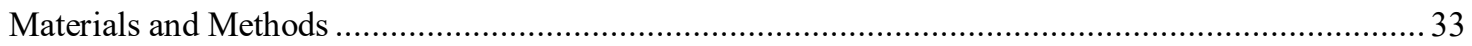

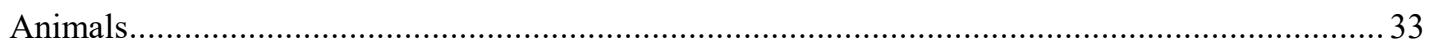

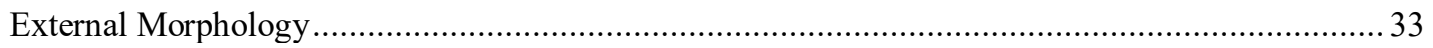

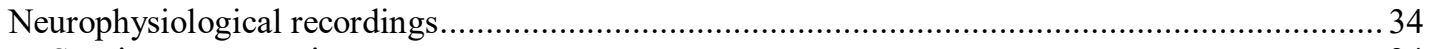

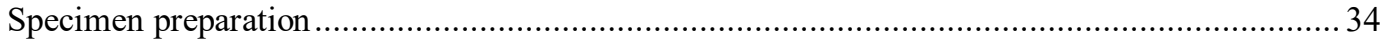

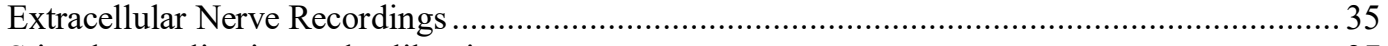

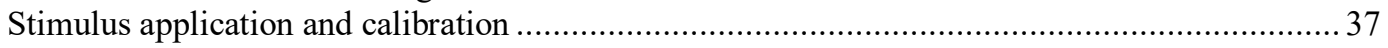

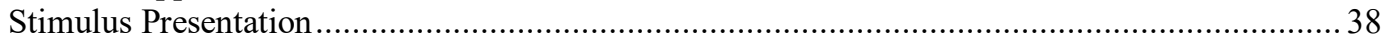

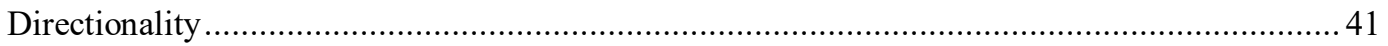

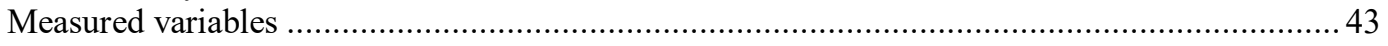

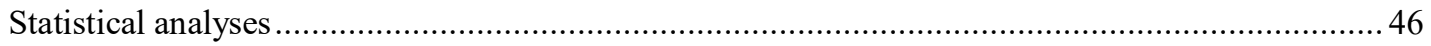

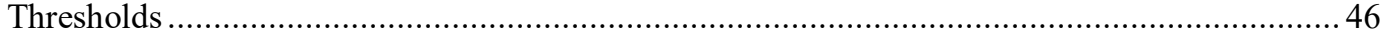

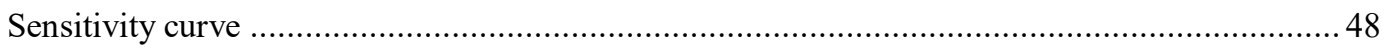

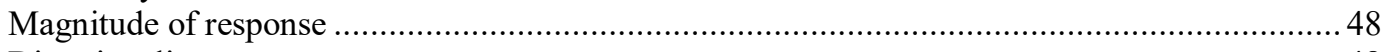

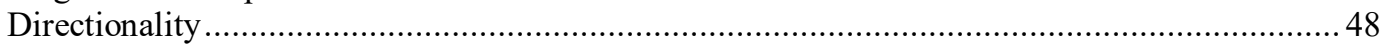

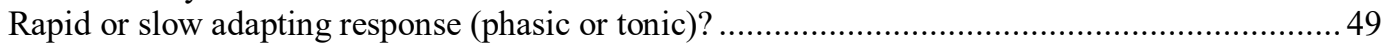

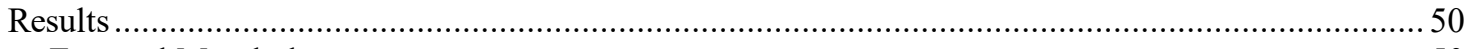

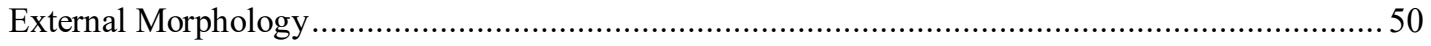

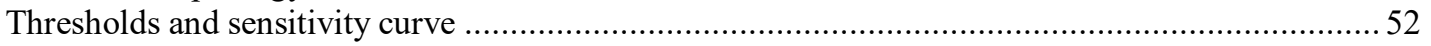

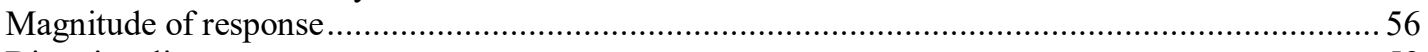

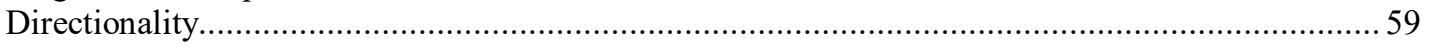

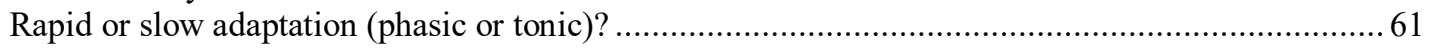

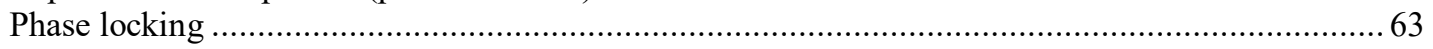

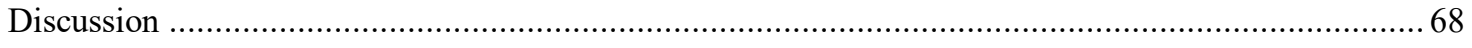

Chapter 3 Vibration detection in Drepana arcuata: insights from one caterpillar ............... 71

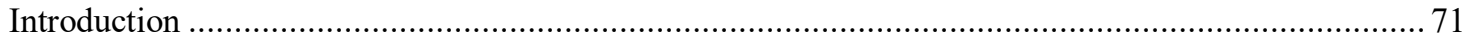

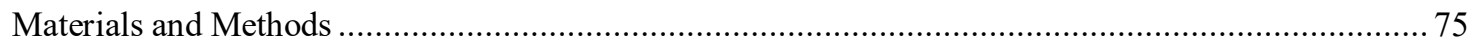

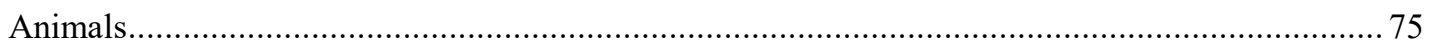

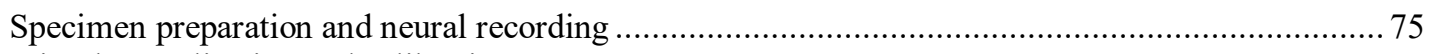

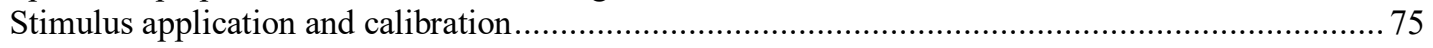




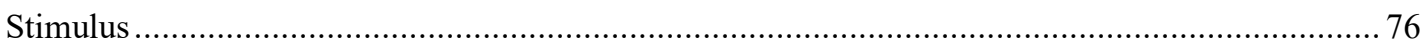

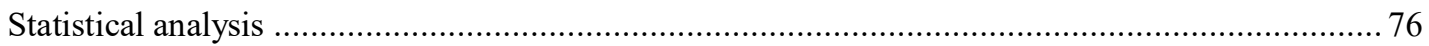

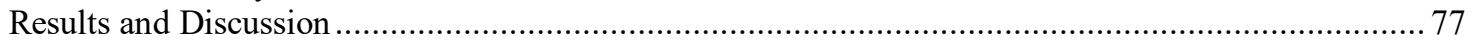

Chapter 4 Tutorial for dissecting, exposing and recording from ventral nerve in the abdominal ganglion of Drepana arcuata ............................................................................................. 80

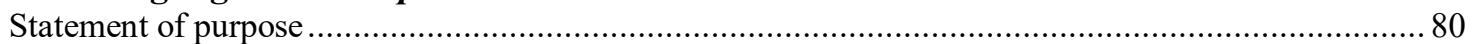

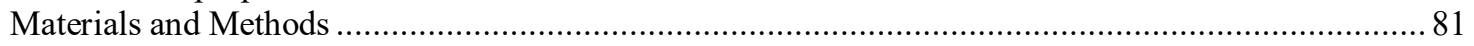

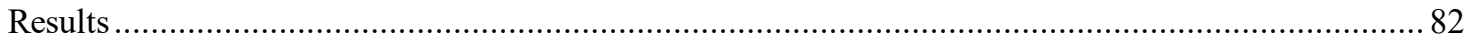

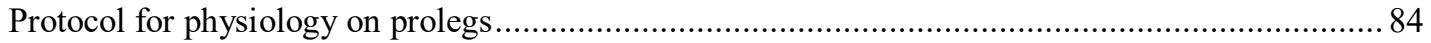

Chapter 5 Final remarks .............................................................................................................90

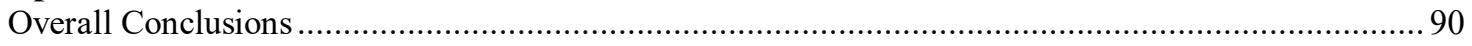

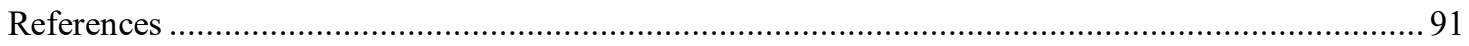

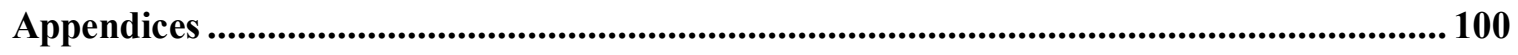




\section{List of Tables}

Table 1.1 - Main characteristics of vibrations measured on plant leaves. ........................................22 


\section{List of Illustrations}

Figure 1.1 - Complex waves that can occur on solid media interfacing with air 4

Figure 1.2 - A vibrational sine wave shown in its different proxies of amplitude. ..............................

Figure 1.3 - Three sine waves with respective frequencies of 20,40 and $80 \mathrm{~Hz}$..............................8

Figure 1.4 - Three sine waves with two-fold increasing frequencies. .............................................9

Figure 1.5 - An illustration of a TRP channel ............................................................................... 12

Figure 1.6 - A diagram showing a hair-like mechanoreceptor, or sensory hair, ............................... 15

Figure 1.7 - Thresholds of leg vibration receptors from three neuropteran species. ..........................19

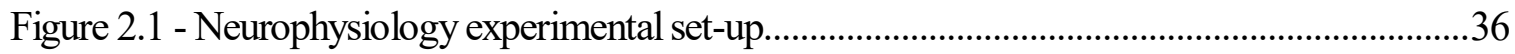

Figure 2.2 - An example of sine vibrational signal as measured by the laser vibrometer...................39

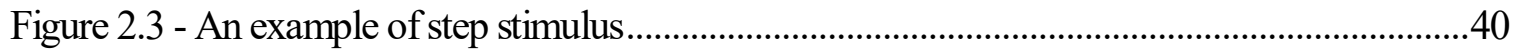

Figure 2.4 - Ventral view of a $T$. ni caterpillar abdomen for directional experiment.........................42

Figure 2.5 - An example trace showing detected spikes................................................................4

Figure 2.6 - Angles assigned to spikes for phase locking analysis. .................................................45

Figure 2.7 - Local regressions loess $($ for $80 \mathrm{~Hz}$ frequency in all $T$. $n i$ caterpillars. ...........................47

Figure 2.8 - T. $n i$ caterpillar resting on a leaf ...................................................................................

Figure 2.9 - Example of traces recorded from a de-efferented nerve. .............................................53

Figure 2.10 - Sensory responses to stimuli of different intensities in $T$. $n i$......................................54

Figure 2.11 - Average thresholds to different frequencies on the velocity proxy ...............................55

Figure 2.12 - Magnitude of response (\# action potentials) to increasing stimulus velocity..............57

Figure 2.13 - Magnitude of response (\# action potentials) at supra-threshold stimuli of $2 \mathrm{~mm} / \mathrm{s} . . . .58$

Figure 2.14 - Diagram representing the four orientations in which step stimuli were applied. .........60 
Figure 2.15 - The sensory hair shows a rapid adaptation to the stimulus. .62

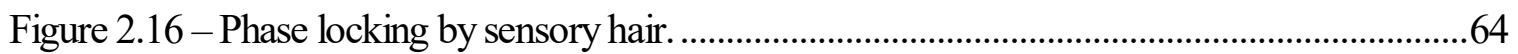

Figure 2.17 - Phase locking for $90,100,110$ and $120 \mathrm{~Hz}$ in one $T$. $n i$ caterpillar.............................66

Figure 2.18 - Phase locking for $80 \mathrm{~Hz}$ in all $\mathrm{T}$. $n i$ caterpillars..........................................................67

Figure 3.1 - In D. arcuata, proleg hairs are often in direct contact with the leaf surface...................73

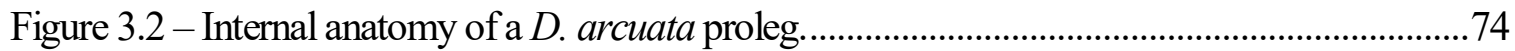

Figure 3.3 - Sensory responses to vibration by the waxed cuticle and hair of $D$. arcuata................. 78

Figure 3.4 - Thresholds of neural response obtained from the two D. arcuata caterpillars...............79

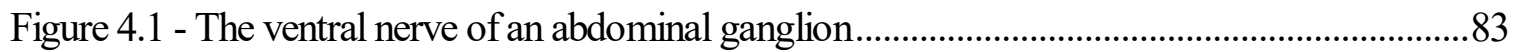

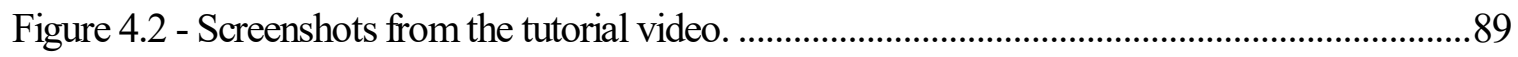




\section{List of Appendices}

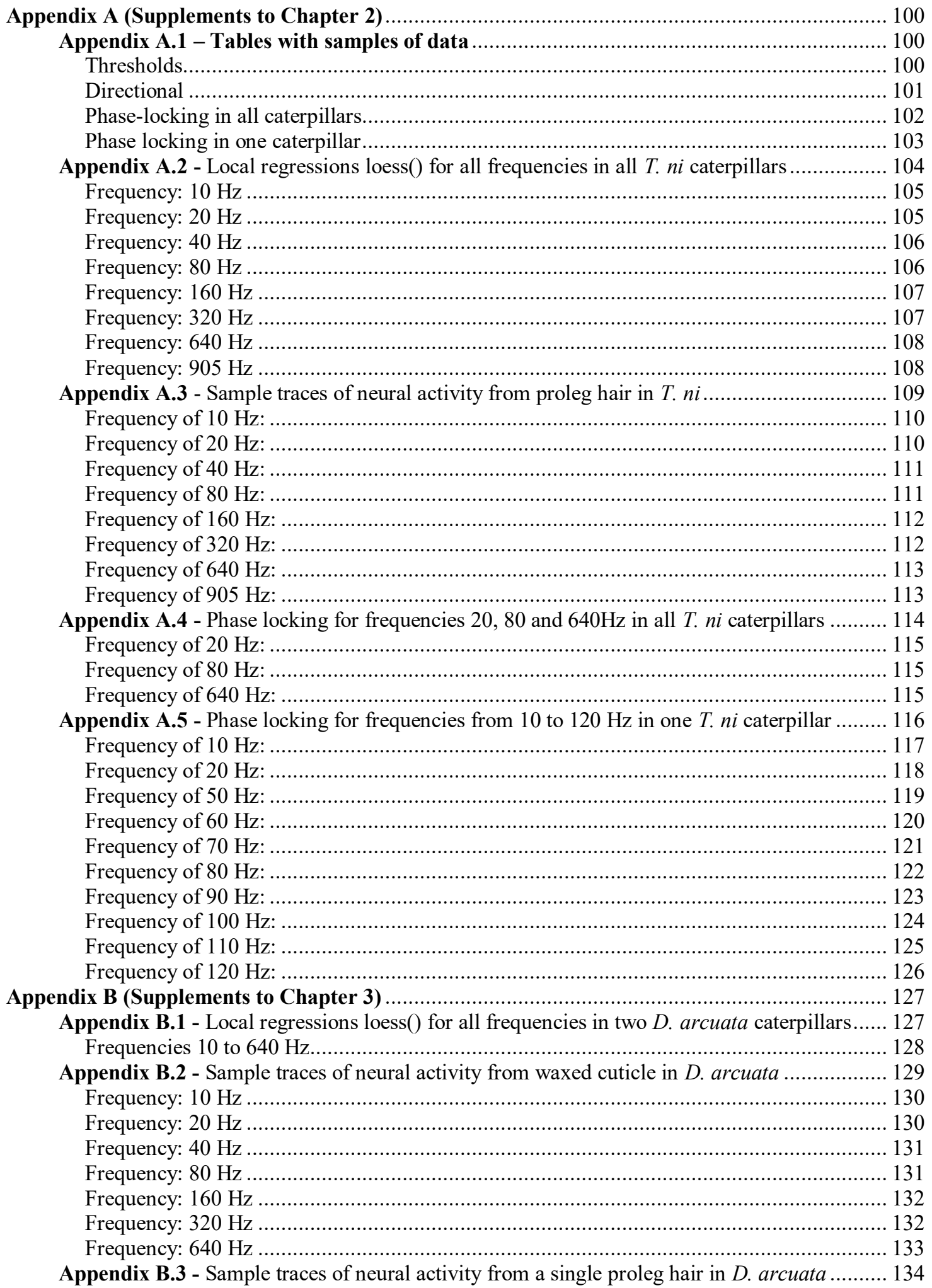




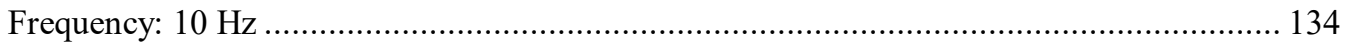

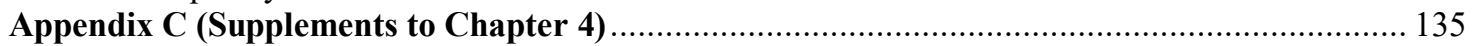

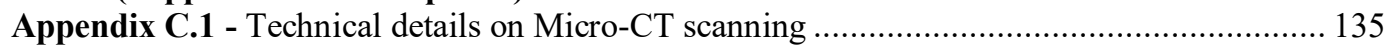

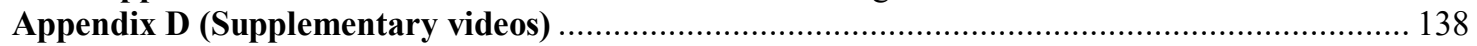

Appendix D.1 - Short tutorial for exposing a caterpillar`s nervous ganglion .................................. 139

Appendix D.2 - Neural activity recorded from ventral nerve ......................................................... 141

Appendix D.3 - What might happen when the caterpillar wiggles ............................................... 142

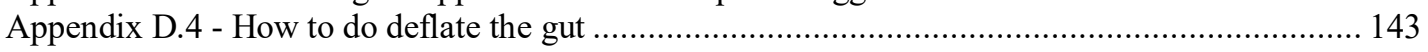

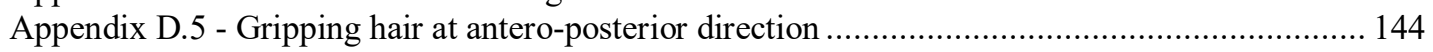

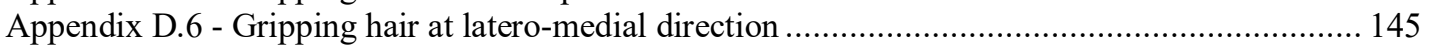

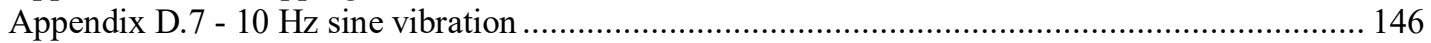

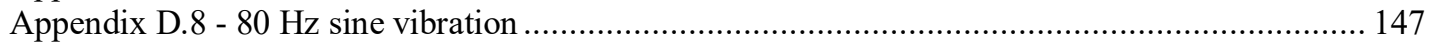

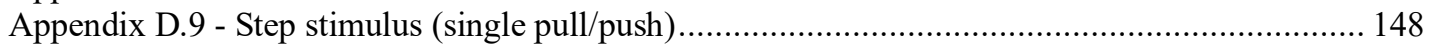




\section{Chapter 1 General Introduction}

\section{What is this thesis about?}

The focus of this thesis is to explore whether Cabbage Looper caterpillars, Trichoplusia ni (Hübner, 1802), and Masked Birch caterpillars, Drepana arcuata (Walker, 1855), can detect substrate-borne vibration through a sensory cuticular hair on their proleg. As humans, we can detect and discriminate substrate-borne vibrations applied to the whole body in a large scale (Griffin, 2012) and to specific points on the skin, in a fine scale (Järvilehto et al., 1976; Konietzny and Hensel, 1977; Vedel and Roll, 1982; Mahns et al., 2006). But humans are not as sensitive to substrate-borne vibration as some other animals (Hill, 2009), including insects (Cocroft and Rodríguez, 2005; Yack 2016). In the next paragraphs of this introduction, fundamental concepts that are necessary to the understanding of this thesis will be introduced, while the following chapters will describe my experiments and results.

\section{What is substrate-borne vibration?}

The physics concepts underlying generation and propagation of mechanical waves, or vibrations, can be hard to understand (Wittmann et al., 2012). Mechanical waves start in a material medium when a disturbance accelerates and displaces particles from their original position, relative to their neighbours (Auld, 1973). During this process, elasticity or other restoring forces that exist between matter particles function

like microscopic springs, de-accelerating them and accumulating potential energy (Auld, 
1973; Fahy and Gardonio, 2007). As a consequence, the potential energy is released to their neighbours by setting them to move along (Auld, 1973; Fahy and Gardonio, 2007). This originates mechanical waves: cyclic motion of individual particles that transfer energy to their neighbours, which in turn end up following similar cyclic motion patterns (Auld, 1973; Fahy and Gardonio, 2007).

Mechanical waves can occur in any material medium including gas, liquid or solid, and adopt several different cyclic motion and propagation kinematics (Fahy and Gardonio, 2007). For instance, the particle displacement can be either perpendicular or parallel to the wave propagation, in which cases waves are categorized as transverse or longitudinal, respectively (Fahy and Gardonio, 2007). Transverse waves are prominent in solids due to the shear forces between particles in this sort of medium, but they are practically irrelevant in fluids, where they have a rapid decay over distance (Fahy and Gardonio, 2007). Longitudinal waves, on the other hand, are not limited to solids: They also occur in fluid media, where they are generally referred to as sound and have the restoring forces greatly driven by pressure differences (Fahy and Gardonio, 2007). However, "simple" models for longitudinal and transverse waves, as if they were not complicated enough, do not apply to all cases.

Several interactions arise when different media interface with each other, originating waves that cannot be categorized as either longitudinal or transverse and other mechanical phenomena that are not quite defined as waves (Fahy and Gardonio, 2007). For instance, more complex kinematics occur in solids interfacing with air and on the air region in proximity to vibrating solids, called bending and quasi-longitudinal waves (Figure 1.1) and near-field sound, respectively (Fahy and Gardonio, 2007). However, 
discussing the physical details of transverse, longitudinal, bending and quasi-longitudinal waves as well as near- and far-field sounds is beyond the scope of this thesis. So, for simplification purposes, mechanical waves will be referred to here simply by the medium in which they occur, i.e. either as "substrate-borne vibration" or "air-borne sound" (e.g Cocroft and Rodríguez, 2005; Yack, 2016). Hence, the term "substrate-borne vibration" used in this thesis refers simply to the resultant cyclic motion performed by particles in a solid medium, regardless of its motion kinematics, the mechanical waves that interfered to create it or where the initial disturbance occurred. 

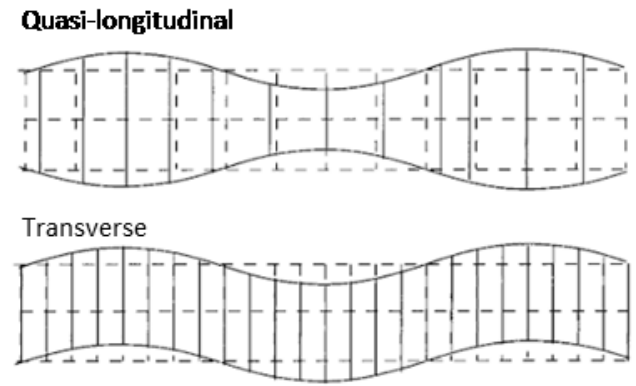

Bending

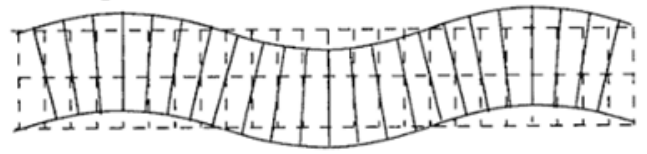

- - - Undeformed _ Deformed

Figure 1.1 - Complex waves that can occur on solid media interfacing with air. From top to bottom, the three diagrams represent quasi-longitudinal, transverse and bending waves, respectively. Notice that for quasi-longitudinal and bending waves rectangles are shown to contract and expand in both directions, parallel and perpendicular to the surface of the solid, which means that particles within them are displacing in both directions simultaneously (modified from Fahy and Gardonio, 2007). 


\section{What physical parameters are important to characterize substrate-borne vibrations?}

As previously explained, mechanical waves are the result of cyclic motion performed by individual particles in a material medium. For most cases, the complexity of vibrations can hardly be fully described by individual parameters or represented in two-dimensional graphs. This is what happens, for example, in quasi-longitudinal waves

on the surface of solid media, whose particles` motion happens in multiple orthogonal directions (Fahy and Gardonio, 2007). However, describing mechanical waves becomes easier in cases that are limited to uni-directional motion, for example as in pure longitudinal or transverse waves (Fahy and Gardonio, 2007). Probably the easiest situation to be characterized is the Simple Harmonic Motion of particles, whose wave can be fully explained by a sine function of time (Fahy and Gardonio, 2007; Griffin, 2012). For this reason, this is the motion pattern that was chosen to be used as the main mechanical stimulus in this thesis.

Uni-directional sine waves can be fully described by only three parameters: Frequency, magnitude, and phase (Griffin, 2012). While frequency and phase are relatively simple measurements, magnitude may be a source of confusion since it can be expressed in three different kinematic quantities that are convertible into each other: Displacement, velocity and acceleration (Figure 1.2, Figure 1.3, Figure 1.4) (Griffin, 2012). The relevance of each of these parameters to insects` sensory systems will be later addressed in this introduction. Common magnitude measurements for these quantities are peak-to-peak displacement, peak velocity and peak acceleration (Figure 1.2, Figure 1.3, Figure 1.4) (Griffin, 2012). These three measurements will be important throughout this 
thesis, as they will help in describing the sensitivity of the mechanoreceptors that I explored.

The magnitude of air-borne sounds can be also expressed using these three kinematic quantities (Fahy and Gardonio, 2007), which is relatively simple when airborne sound propagates as longitudinal waves and the particles motion is uni-directional (Fahy and Gardonio, 2007). However, the magnitude of air-borne sounds is more often expressed as pressure, which is also convertible in either displacement, velocity or acceleration (Fahy and Gardonio, 2007). In fact, the pressure quantity in air-borne sound has a direct relationship with particle velocity (Fahy and Gardonio, 2007; Ter Hofstede et al., 2011). This preference in using pressure to express magnitude of air-borne sound is probably due to this quantity being more directly measured by artificial transducer equipment. As I will explain below, many insect structures that detect air-borne sounds also work as pressure instead of movement receivers (Michelsen, 1979). However, since this thesis focuses on substrate-borne vibrations, I will characterize their amplitude using the three kinematic quantities mentioned above, specifically as peak acceleration, peak velocity and peak-to-peak displacement (Figure 1.2). Moreover, since the vibrations tested here consist of uni-directional sine waves, their frequency and phase, in addition to their amplitude, will be enough to fully characterize them. 

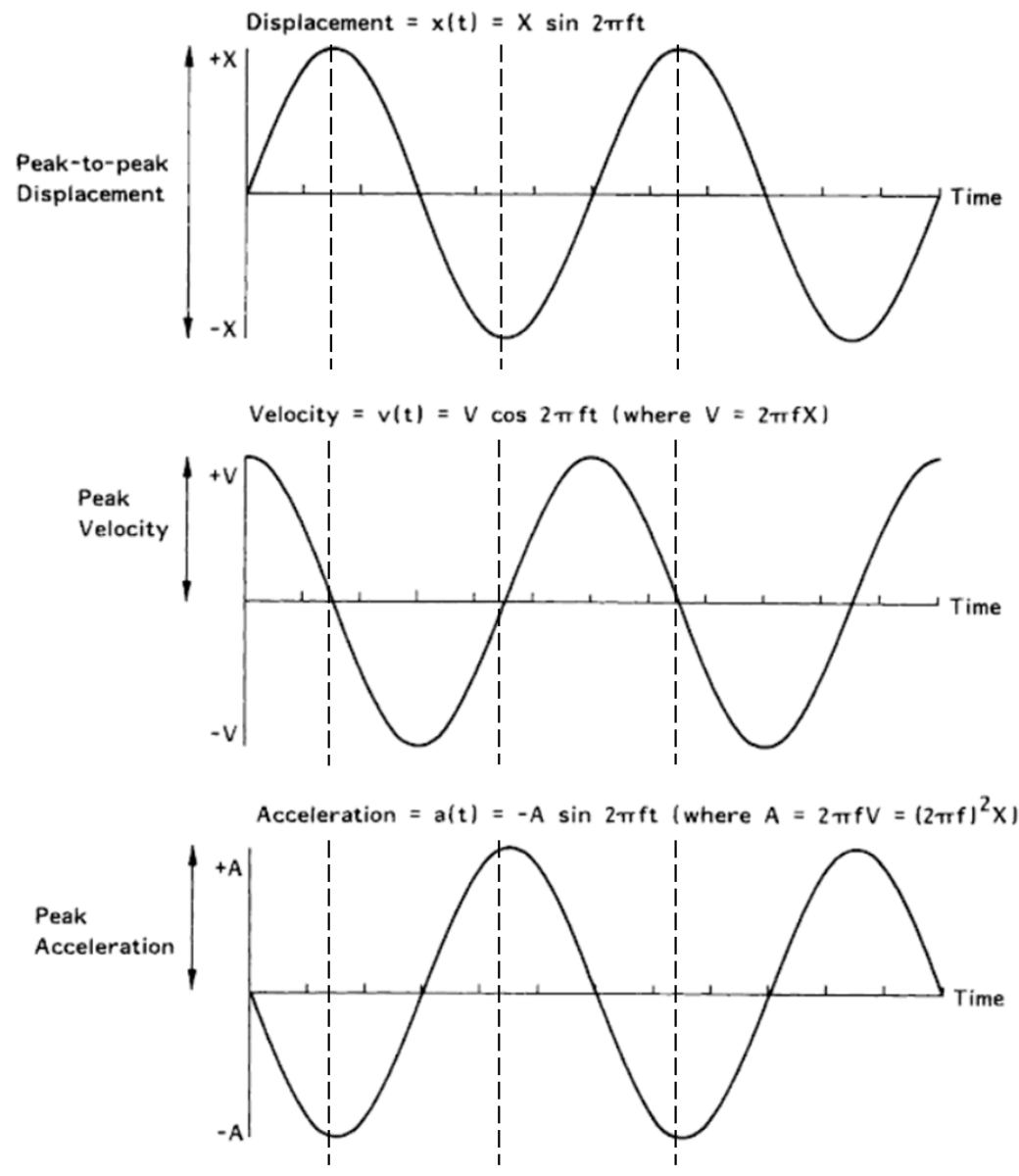

Figure 1.2 - A vibrational sine wave shown in its different proxies of amplitude. Dashed lines help indicate phase relationships that occur between the proxies when they are converted into each other. Arrows on the left side indicate most common magnitude measurements, which were also used throughout this thesis. Equations to convert peak displacement $(\mathrm{X})$ to peak velocity $(\mathrm{V})$ and peak acceleration $(\mathrm{A})$ are shown on the velocity and acceleration graphs: $V=2 \pi f X$ and $A=(2 \pi f)^{2} X$. Notice that the wave frequency $(f)$ is a factor in both equations. Because of this, if two sine waves have the same peak displacement but different frequencies, their peak velocities and accelerations will necessarily be different, and vice versa. The consequence of this mathematical relationship is better observed in Figure 1.3 and Figure 1.4 (adapted from Griffin, 2012) 


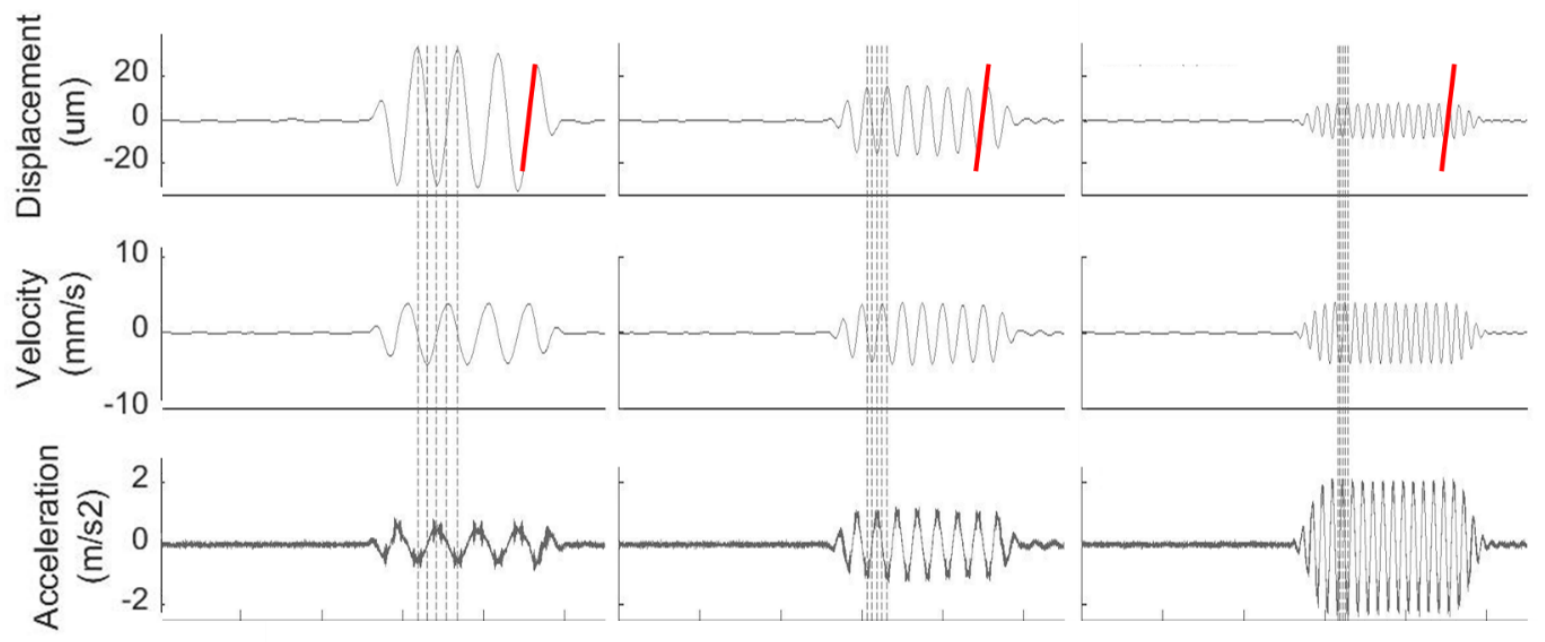

Figure 1.3 - Three sine waves with respective frequencies of 20,40 and $80 \mathrm{~Hz}$ (left to right) shown in its different proxies of amplitude (top to bottom). Dashed lines indicate the phases of a full cycle. Red bars on top windows indicate the maximum slope of the displacement waves. These waves consist of actual substrate-borne vibrational signals programmed, applied and measured by myself. The three waves have the same approximate peak velocity, of $4.0 \mathrm{~mm} / \mathrm{s}$, but not the same frequency and hence neither the same peak displacement (top windows) or acceleration (bottom windows). Another way to interpret this relationship is by comparing the slopes of the waves: the maximum slope of a wave shown in the displacement proxy corresponds to its peak-velocity. On the top windows, for example, the waves may have different peak-displacements, but they end up having the same maximum slope because their frequencies are inversely proportional and thus have the same peak-velocity. 

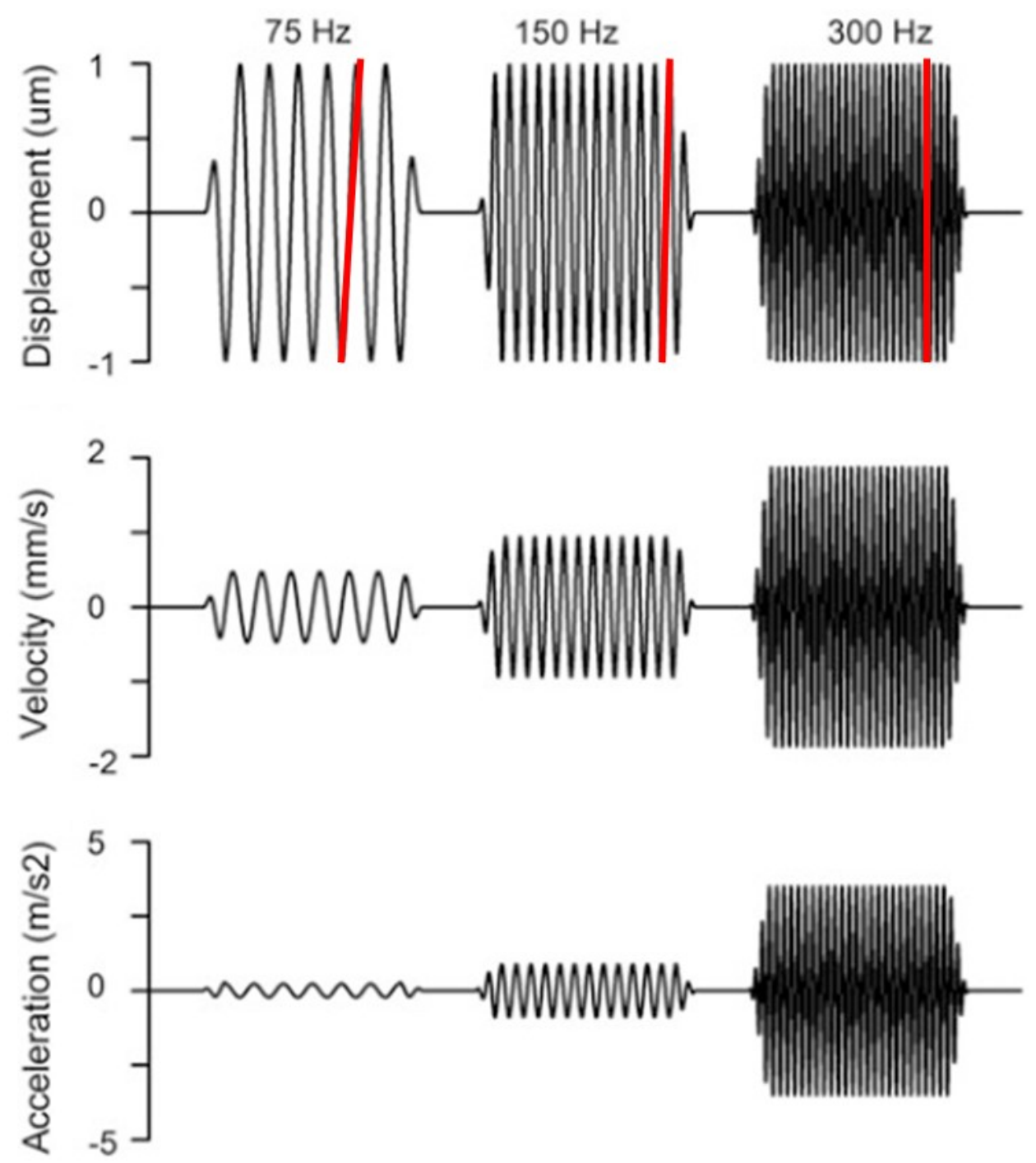

Figure 1.4 - Three sine waves with two-fold increasing frequencies. The difference from Figure 1.2 is that here the waves were set to have the same peak displacement. As a consequence, they must have different peak velocities and accelerations. Red bars on top windows indicate slopes in the displacement proxy (adapted from Cocroft et al., 2014) 


\section{What is the transduction mechanism of mechanical waves in the molecular level?}

Although the mechanism for transducing mechanical waves into neural signals is not completely understood, there has been great progress in recent years $\left(\mathrm{O}^{`} \mathrm{Neil}\right.$ and Heller, 2005; Lumpkin et al., 2010; Bokolia and Mishra, 2015). For instance, it is widely accepted that the molecular mechanisms used to transduce general mechanical stimuli are fundamentally the same for most cell types in most living beings, from membrane forces in bacteria to touch and hearing in humans (Lumpkin et al., 2010). This also applies to non-sensory cells that detect mechanical stresses on the cell membrane in vertebrates (O`Neil and Heller, 2005) and to sensory cells that detect air-borne sound, substrateborne vibration and touch in insects (O`Neil and Heller, 2005; Lumpkin et al., 2010; Bokolia and Mishra, 2015).

The latency times of mechanosensory cells can be very short, lower than 1.0 millisecond (O`Neil and Heller, 2005; Lumpkin et al., 2010). This supports the hypothesis that the fundamental transduction mechanism does not involve any intermediate messenger systems (O`Neil and Heller, 2005), but is rather performed by ion channels that are directly gated by mechanical stresses, such as stretch, shear, deflection or indentation (Lumpkin et al., 2010; Bokolia and Mishra, 2015; Katta et al., 2018; Bavi et al., 2018). However, there are contrasting hypotheses about whether the ion

channels receive stress from the surrounding phospholipids in the cell membrane or if tethered filaments of the cytoskeleton and the extracellular matrix are responsible for redirecting the stress (Lumpkin et al., 2010).

Nevertheless, several ion channels that respond to mechanical stimuli have been identified, some of which belong to molecular families that have homologous 
representatives in both invertebrates and vertebrates (O`Neil and Heller, 2005). Two important groups are the transient receptor potential channels (TRP channels) (Figure 1.5) and the degenerins/epithelial sodium channels (DEG/ENaC), although their specific roles in vertebrates remain to be confirmed (O'Neil and Heller, 2005; Lumpkin et al., 2010). Talking specifically about insects, ion channels from the TRP superfamily were shown to be important for mechanical reception in Drosophila melanogaster (O`Neil and Heller, 2005; Lumpkin et al., 2010; Bokolia and Mishra, 2015) including for touch detection by sensory hairs and proprioception and hearing by chordotonal organs (Lumpkin et al., 2010), a very important anatomical structure that will be further explained below. In fact, two pesticides whose mechanism of action had been unknown were just recently shown to disrupt the functioning of two TRP channels in chordotonal organs, thereby greatly disturbing hearing to similar levels as mutants whose genes for those TRP channels had been silenced (Nesterov et al., 2015). Altogether, this evidence shows that we are getting closer to describing transduction mechanisms for mechanical waves, although we are far from explaining which mechanical stresses the ion channels can receive and how they transduce each of them (e.g. Katta et al., 2018; Bavi et al., 2018). 


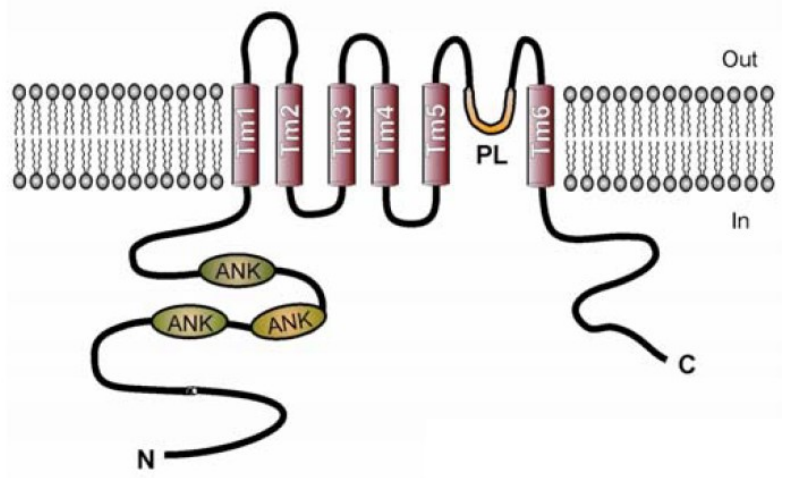

Figure 1.5 - An illustration of a TRP channel showing the pore loop (PL) through which ions will pass when the channel is gated by mechanical stress (adapted from $\mathrm{O}^{`} \mathrm{Neil}$ and Heller, 2005). 


\section{What cell types and sensilla are involved in mechano-transduction in insects?}

Mechanoreceptor sensilla in insects can vary a lot in their shape, size and specific function (Keil, 1997). Most of them however can be grouped into three broad categories: hair-like sensilla, campaniform sensilla or scolopidia (Keil and Steinbrecht, 1984; Keil, 1997). Many hair-like sensilla serve as olfactory receptors (Keil and Steinbrecht, 1984), but in this thesis I will refer specifically to hair-like sensilla that have mechanoreceptor roles.

The cellular organization of most hair-like and campaniform sensilla is very similar (Figure 1.6), with more noticeable variations occurring only on their external morphology (Keil and Steinbrecht, 1984; Keil, 1997). All of them have in common that they are innervated by the dendrite of one bipolar neuron and surrounded by the same types of auxiliary cells (Figure 1.6) (Keil, 1997). Ion channels sensitive to mechanical stimuli are located on this dendrite (Yack, 2004; Lumpkin et al., 2010; Bokolia and Mishra, 2015). Scolopidia, on the other hand, have distinguishable characteristics from the hair-like and campaniform sensilla. For instance, some ion channels on the dendrites of scolopidia are unique and cannot be found in the dendrites of other mechanoreceptor in insects (Nesterov et al., 2015). Scolopidia also occur with varying numbers of innervating bipolar neurons, different types of surrounding auxiliary cells and different forms of attachment to the cuticle - or organ - where they serve as mechanoreceptors (Keil, 1997; Yack, 2004). Moreover, scolopidia form chordotonal organs (Yack, 2004; Yack, 2016), which will be later mentioned in this thesis as an important component of more elaborate mechanoreceptors such as the Johnston`s and the subgenual organs (Yack, 2016). 
There are plenty of different names used in the literature to refer to hair-like sensilla, some of which try to best characterize their morphological or functional aspects. For example, sensory hair, filiform hair, tactile hair, thread-hair, hair-plate, tactile seta, trichoid sensillum, trichobothrium and bristle are different names found in the literature that refer to insect mechanoreceptors that include hair-like structures on the cuticle (e.g. Thurm, 1965; Gnatzy, 1976; Dumpert and Gnatzy, 1977; Tautz, 1977; Keil and Steinbrecht, 1984; Kent at al., 1996; Keil, 1997; Yack, 2004; Rota and Wagner, 2008). Some of these names are used more consistently than others to refer to hair-like structures with a specific morphology or function. For example, bristle usually refers to tactile receptors (Keil, 1997; Duggan, 2000) and trichobothrium usually refers to mechanoreceptors detecting air currents or air-borne sound (Keil, 1997; Duggan, 2000), sensory hair is used in a broader context to refer to general sensory hair-like structures (Tautz, 1977; Tautz, 1978). In this thesis, I chose to use the term "sensory hair" to refer to the studied hair-like structure since its specific function remains to be confirmed. 


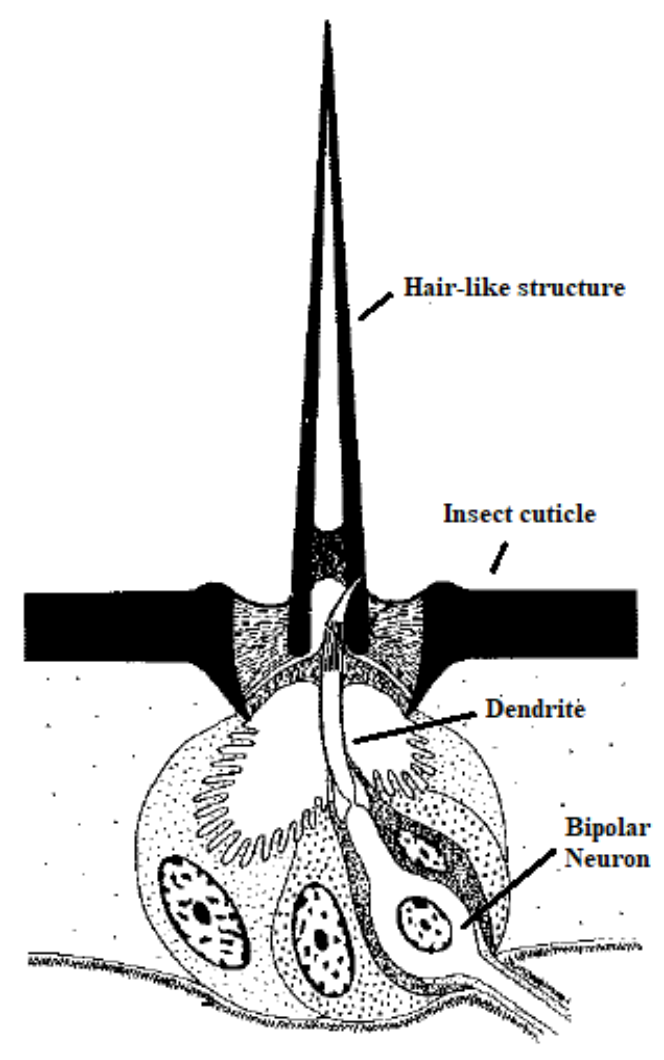

Figure 1.6 - A diagram showing a hair-like mechanoreceptor, or sensory hair, with the basic structures and general cell types. The dendrite of the bipolar neuron is responsible for receiving mechanical stress and transducing it into neural signals. This process involves mechanical stress sensitive ion channels such as the TRP channel shown in Figure 1.5 (adapted from Keil and Steinbrecht, 1984). 


\section{What physical parameters of mechanical waves do insects` sensors receive and transduce into neurophysiological responses?}

As previously described, during the propagation of mechanical waves, particles adopt a cyclic motion, whose magnitude can be measured as displacement, velocity or acceleration for substrate-borne vibrations and pressure for air-borne sounds. But how are these parameters received by insects` organs? For example, hearing organs, i.e. organs that detect air-borne sounds, can be categorized as either pressure or movement receivers (Michelsen, 1979). Movement receivers generally consist of insects` antennae or hair-like sensilla, which can be loosely attached to the cuticle and follow the vibration of air particles (Michelsen, 1979).

Pressure receivers are broadly represented by physical structures with a large area and low weight such as tympanal membranes and balloon-shaped palps (Michelsen, 1979). Although pressure is the physical parameter that is primarily received by tympanal membranes, it is not the parameter directly transduced into neural signals (Ter Hofstede et al., 2011; Montealegre-z et al., 2012). In this regard, tympanal membranes in insects serve to transduce pressure waves into movement, which in turn can be detected by scolopidia that are directly attached to the inner surface of the tympanum (Yack, 2004; Montealegre-z et al., 2012) or to secondary internal structures (Montealegre-z et al., 2012). Therefore, mechanoreceptors ultimately detect kinematic parameters of movement, such as displacement, velocity and acceleration (Figure 1.7).

In fact, this has implications that can be observed in both the sensitivity thresholds of these mechanoreceptors and the stimulus phases at which they trigger action potentials. For instance, the scolopidia attached to tympanal membranes of moths (Yack, 
2004) have equal displacement thresholds across sound frequencies, meaning that they trigger action potentials whenever the tympanum is displaced at a certain amplitude, regardless of the particle velocity (Ter Hofstede et al., 2011). This pattern is not limited to pressure receivers: sensory hairs that function as movement receivers of air-borne sound in the thorax of caterpillars also have equal displacement thresholds across frequencies from 150 to $1000 \mathrm{~Hz}$ (Tautz, 1978). This tendency expands to other cases described in the literature in which sensory hairs are not necessarily displacement receptors. For instance, hairs on the cerci of crickets, which also respond to air-borne sound in the near-field, can be either velocity or acceleration receptors, situations in which thresholds are equal across all tested frequencies (Shimozawa and Kanou, 1984). In addition, these sensory hairs present some sort of phase locking, which can happen for displacement (Tautz, 1978), velocity and acceleration receptors (Shimozawa and Kanou, 1984). This evidence suggests that mechanical waves can be detected by sensory hairs that respond consistently to any of the three quantities representative of vibration magnitude, i.e. displacement, velocity and acceleration.

Mechano-sensitivity to one of the three kinematic quantities is not limited to airborne sounds. Similar results have been observed in organs that are involved in detecting substrate-borne vibration (Figure 1.7), such as the femoral chordotonal organ and the subgenual organ, whose basic mechanoreceptors are scolopidia (Yack, 2016). For example, the femoral chordotonal organ of a stick insect species was shown to possess different scolopidia that are independently sensitive to either velocity or acceleration, with neural responses occurring accordingly at specific phases of the stimuli (Hofmann et al., 1985; Hofmann and Koch, 1985). Regarding the subgenual organ, honeybees and a 
stick insect species were shown in two independent studies to have equal thresholds across frequencies for velocity and acceleration, respectively (Cocroft, 2016; Strauß and Lakes-Harlan, 2017). Moreover, equal sensitivity thresholds to substrate-borne vibrations are also found in the displacement quantity: equal displacement thresholds as well as acceleration thresholds were found in leg mechanoreceptors of a neuropteran (Figure 1.7) (Devetak, 1998) and a hemipteran (Čokl, 1983). In these studies, it was not clear which organs in the leg had originated the neural responses, but they were arguably subgenual organs, chordotonal organs and maybe campaniform sensilla (Čokl, 1983; Devetak, 1998). Therefore, insect receptors that transduce information from mechanical waves can detect one out of these three physical quantities, although the molecular mechanisms for transducing these parameters are not known yet. 


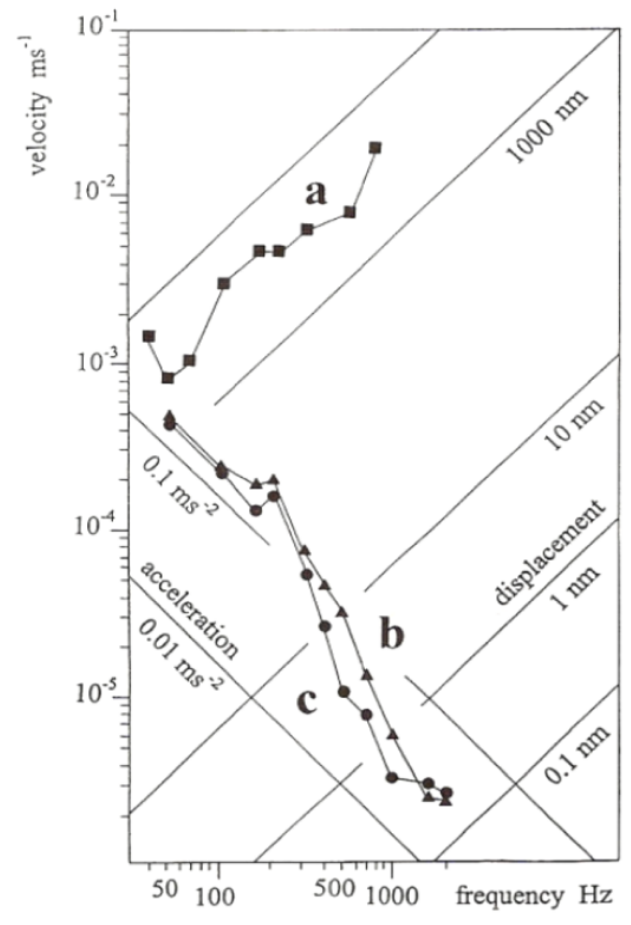

Figure 1.7 - Thresholds of leg vibration receptors from three neuropteran species. Each threshold line, indicated by letters a, b and c, represents one species. While the vertical axis is given in the velocity proxy, the figure also shows calculated isolines for the other two proxies of vibration magnitude, i.e. displacement and acceleration. Notice that isolines for these proxies are diagonal to the vertical and horizontal axis. This happens because sine waves of the same peak velocity will necessarily have different peak accelerations and displacements if their frequencies are different, and vice versa. Therefore, an acceleration or displacement isoline cannot be horizontal, which would mean waves with different frequencies. This phenomenon was illustrated in Figure 1.3 and Figure 1.4 as well. In this figure, it can be observed that leg receptors in each species have thresholds that follow different isolines: displacement for species a and acceleration for species b and c. (adapted from Devetak, 1998). 


\section{What is the physical profile of substrate-borne vibrations in plant leaves?}

Substrate-borne vibrations propagating in leaves are extremely complex for many reasons (Tang et al., 1986; Magal et al., 2000; Fariñas et al., 2012; Cocroft, 2016). First, all the distinct wave categories that had been previously mentioned, such as transverse, longitudinal, quasi-longitudinal and bending waves, occur simultaneously in leaves (Fariñas et al., 2012; Cocroft, 2016). Second, leaves have their own resonance modes for both transverse and longitudinal waves - which can vary depending on leaf properties and size - and affect resultant vibrations (Tang et al., 1986). Third, plant leaves have a structure that is highly heterogeneous, which affects the characteristics of waves as they propagate through the medium (Magal et al., 2000; Fariñas et al., 2012). For example, waves attenuate differently when they propagate through distinct anatomical parts such as veins or parenchyma (Magal et al., 2000) and their propagation speed differs between the adaxial and the abaxial sides due to differences in cellular organization and stomatal crypt distribution (Fariñas et al., 2012).

These differences in propagation velocities, which can range from 250 to $500 \mathrm{~m} / \mathrm{s}$ depending on the leaf side for longitudinal waves (Fariñas et al., 2012) do not affect only the time that it takes for waves to propagate over distances: it also causes variation in the wavelengths, which in turn affects the three-dimensional motion pattern of quasilongitudinal waves and their resultant amplitudes in the parallel and perpendicular axes to the leaf surface (Fahy and Gardonio, 2007; Cocroft, 2016). Therefore, the spectral, temporal and amplitude characteristics of vibrations on a leaf can vary dramatically depending on where the vibration disturbance originally occurred, where the vibration 
was finally measured and everything that was in between (Tang et al., 1986; Magal et al., 2000; Fariñas et al., 2012; Cocroft, 2016).

Another source of complexity arises when we examine the most popular techniques for measuring substrate-borne vibration: Doppler laser vibrometers and unidirectional accelerometers can only detect the amplitude of vibrations happening perpendicular to the leaf surface (Cocroft, 2016). However, as previously discussed, the waves on these media have a quasi-longitudinal motion pattern, which means that a great proportion of their energy and movement happens parallel to the surface (Fahy and Gardonio, 2007; Cocroft, 2016). For example, a simplified calculation revealed that a quasi-longitudinal wave of $1000 \mathrm{~Hz}$ propagating at $500 \mathrm{~m} / \mathrm{s}$ through a $5 \mathrm{~mm}$ diameter rod shaped object, such as a plant stem, would cause particles on the surface to displace in the parallel direction with amplitudes 100 times greater than in the perpendicular direction (Cocroft, 2016).

Therefore, measured vibrations on the leaves might have important amplitude components on directions that we cannot measure by general methods, but that insects might be able to perceive (Cocroft, 2016). In addition, very few studies have characterized vibrations that are reportedly important to caterpillars (e.g. Yack et al., 2001; Castellanos and Barbosa, 2006; Bowen et al., 2008; Guedes et al., 2012), which adds more uncertainty to the types of vibrations that caterpillars can actually detect. Nevertheless, we can use amplitude values provided in the literature as a reasonable starting point to plan experiments. Table 1.1 shows amplitude values that result from occasional activities in one caterpillar species, predators and abiotic factors that aided me in planning my experiment. 
Table 1.1 - Main characteristics of vibrations measured on plant leaves. These signals were measured on leaves of Paper Birch, Betula papyrifera, under the effect of different biotic and abiotic factors, as indicated by the red rectangle. Biotic factors correspond to vibrations generated by caterpillars of Drepana arcuata and adults of Podisus maculiventris. Signals were measured by a Doppler Laser Vibrometer pointed perpendicularly at the leaf surface, which means that parallel components of particles movements were not measured (adapted from Guedes et al., 2012).

\begin{tabular}{llcll}
\hline $\begin{array}{l}\text { Source of } \\
\text { vibration }\end{array}$ & $\begin{array}{l}\text { Periodicity } \\
\text { (events/s) }\end{array}$ & $\begin{array}{l}\text { Dominant } \\
\text { frequency }(\mathrm{Hz})\end{array}$ & $\begin{array}{l}\text { Velocity } \\
(\mathrm{mm} / \mathrm{s})\end{array}$ & $\begin{array}{l}\text { Distance from } \\
\text { laser spot (mm) }\end{array}$ \\
\hline Resident & & & & \\
$\quad$ Chewing (bites) & $1.69 \pm 0.24$ & $7.94 \pm 2.77$ & $2.47 \pm 1.03$ & $29.82 \pm 1.54$ \\
$\quad$ Anal scraping & $0.84 \pm 0.13^{\mathrm{a}}$ & $38.3 \pm 9.40$ & $5.28 \pm 2.84$ & $15.40 \pm 6.73$ \\
Mandible drumming & $1.94 \pm 0.14^{\mathrm{a}}$ & $56.70 \pm 26.30$ & $49.91 \pm 29.25$ & $16.71 \pm 6.60$ \\
$\quad$ Mandible scraping & $0.68 \pm 0.42^{\mathrm{a}}$ & $11.7 \pm 5.60$ & $29.02 \pm 17.23$ & $19.33 \pm 4.51$ \\
\hline $\begin{array}{l}\text { Intruder } \\
\text { Caterpillar crawling }\end{array}$ & $10.70 \pm 5.77$ & $7.47 \pm 4.38$ & $2.08 \pm 1.78$ & $21.5 \pm 9.71$ \\
Predator walking & $9.40 \pm 6.70$ & $11.7 \pm 6.80$ & $3.26 \pm 2.02$ & $23.10 \pm 5.87$ \\
Abiotic & & & & $\mathrm{n} / \mathrm{a}$ \\
Wind & $4.14 \pm 1.56^{\mathrm{b}}$ & $5.02 \pm 1.18$ & $198.4 \pm 28.40$ & $\mathrm{n} / \mathrm{a}$ \\
Rain (droplets) & $56.60 \pm 5.80^{\mathrm{b}}$ & $55.29 \pm 47.50$ & $127.2 \pm 25.90$ & $20 \mathrm{~mm}$ \\
Ball dropping & $\mathrm{n} / \mathrm{a}$ & $8.46 \pm 6.51$ & $\mathrm{n} / \mathrm{a}$ & \\
\hline
\end{tabular}




\section{After all, what is a substrate-borne vibration receptor?}

Focusing on the insects`sensory systems, the distinction between substrate-borne vibration receptors and air-borne sound receptors is consistent for most practical cases, since the anatomical traits necessary for receiving and transducing these components are generally very different (Michelsen and Nocke, 1974; Michelsen, 1979; Yack, 2004; Montealegre-z et al., 2012; Yack, 2016). This becomes evident for example when tympanal and subgenual organs are compared: the former is necessary for receiving pressure waves from the air and aiding reducing impedance effects between media (Yack, 2004; Montealegre-z et al., 2012), whereas the latter is necessary for detecting tiny movements of the hemolymph in the leg that are caused by inertia during vibrations (Kilpinen and Storm, 1997). Indeed, it is difficult to imagine the opposite: a tympanal organ vibrating due to inertia or a subgenual organ receiving pressure waves from the air.

However, when a solid and a fluid medium interface, mechanical waves originating from the disturbance in one medium can propagate to the other, in either direction (Martens and Michelsen, 1981; Fahy and Gardonio, 2007; Kavčič et al., 2013). The waves that propagate between different media can eventually be detected by animals` sensory systems, especially because waves can also propagate through the animal body itself (Shaw, 1994a; Hill, 2009). For this reason, there are reported cases of vertebrate ears - supposedly responsible for receiving air-borne sound - that detect substrate-borne vibrations, including in humans (Narins and Lewis, 1984; Todd et al., 2008; Hill, 2009). And there are a couple reported cases of subgenual organs in cockroaches and neuropterans - supposedly responsible for detecting substrate-borne vibration - that 
detect air-borne sounds, even in the far-field for cockroaches (Shaw, 1994a; Devetak, 1998).

In these two situations, for the vertebrate ears it is unlikely that the tympanal membrane is involved in transducing the substrate-borne vibrations (Narins and Lewis, 1984), while for the subgenual organ of cockroaches it is also unlikely that they are receiving pressure waves directly from the air (Shaw, 1994a). There must be another path that the mechanical waves are taking through the body to trigger neural response in these sensory organs (Narins and Lewis, 1984; Shaw, 1994a). These are isolated situations, since they differ from most functions traditionally described for these receptors. Moreover, although cockroaches indeed respond behaviorally to air-borne sound, it is not clear whether the subgenual organs are involved at all in this process (Shaw, 1994a). For instance, the neural response to airborne sound could be practically non-functional for the cockroach`s central nervous system. However, facts like these bring up the idea that mechanical waves, whether in the air or in solids, are less distinctive from each other than we may have thought (Hill, 2009).

From a sensory perspective, the distinction between air- and solid-borne waves becomes even less evident when we consider that some mechanoreceptors can detect airborne sound or substrate-borne vibration without remarkable anatomical differences. This is particularly what happens for displacement detectors of air particles such as sensory hairs and the Johnston`s organ in insects`antennae (Michelsen, 1979). For instance, whereas in some insect species the Johnston`s organ is reported to detect air-borne sounds in the near-field when the antenna vibrates with air particles (Ai, 2009), in other species it is thought to detect substrate-borne vibrations when the antenna touches the 
substrate (Jeram and Čokl, 1996; Čokl et al., 1999). This does not mean that antennae used for either function have the exact same morphology, mechano-transduction characteristics and afferent projections in the Central Nervous System - in fact, they likely do not. However, this fact shows that substrate-borne vibrations and air-borne sounds may have enough physical characteristics in common so that mechanoreceptors with relatively similar aspects can detect either - or both.

Regarding sensory hairs, it is known that the thorax of some caterpillars have some hairs that are naturally responsive to near-field air-borne sounds (Tautz, 1977; Tautz, 1978; Tautz and Markl, 1978). However, the same hairs respond to solid-borne vibrations if forced vibrations are applied using an attached needle (Tautz, 1977). - My thesis tests the hypothesis that hairs on caterpillar prolegs can detect vibrations. This is important because while many larval insects detect vibrations, to date there is no direct evidence for how they are doing this (see details in Introduction of Chapter 2). To test this I will do the following:

a. determine if the hair is sensory;

b. determine if it responds to vibration;

c. determine how it responds to vibration;

d. discuss the relevance.

This thesis has two experimental chapters -2 and 3 - each conducting tests in a different species. All experiments in both chapters follow fundamentally the same neurophysiological approach, in which sensory hairs are stimulated by forced vibrations applied by forceps or stick. Most equipment used and analyses executed are also the 
same, although some aspects of the mechanical stimuli differed. Therefore, the similar set-up in both chapters was planned to allow comparisons between both species. Finally, Chapter 4 is a simple tutorial, where a protocol for dissecting and recording from caterpillars is described. The purpose is to guarantee repeatability of the experiments from chapters 2 and 3, facilitating future students to further explore vibration detection in these organisms. 
Chapter 2 Vibration-sensitive hair on the proleg of Trichoplusia ni (Noctuidae): velocity thresholds, directionality and intensity of response

A modified version of this chapter will be submitted as a manuscript to a peer reviewed journal.

Rosi-Denadai C.A., Lewis, J. and Yack J.E. Vibration-sensitive seta on the proleg of Trichoplusia ni (Noctuidae): velocity thresholds, directionality and intensity of response. Journal of Comparative Physiology A. (In preparation) 


\section{Introduction}

One of insects` most important sensory system involves the perception of mechanical vibrations transmitted through air, water or solids (Yack, 2004, Cocroft and Rodríguez, 2005; Yack, 2016). Sensitivity to solid borne vibrations transmitted through plant material is employed for communication and environmental monitoring by insects from at least eighteen orders (Cocroft and Rodríguez, 2005). Despite the widespread occurrence of this sense, the literature primarily reports solid-borne vibration sensitivity by insects in the adult stage, leaving a huge knowledge gap about the earlier life stages of these organisms (Yack, 2016).

There are several examples of juvenile insects of both hemi- and holometabolous groups detecting substrate-borne vibrations (Cocroft, 1999; Yack, 2016). In hemimetabolous insects, treehopper nymphs use vibratory signals to trigger in other nymphs and the mother an anti-predator behaviour. In holometabolous insects, examples include Cerambycidae larvae possibly communicating to each other in the wood (Kočárek, 2009), Scarabaeidae larvae responding to pupae signals in the soil (Kojima et al., 2012a; Kojima et al., 2012b), wasp larvae responding to adult vibratory signals within colonies (Ishay et al., 1974; Savoyard et al., 1998; Cummings et al., 1999), gregarious sawfly larvae coordinating group interaction by tapping signals on the plant (Fletcher, 2007; Fletcher, 2008), Geometridae caterpillars detecting vibratory signals of approaching predators (Castellanos and Barbosa, 2006) and caterpillars using complex vibratory signals for communicating with conspecifics such as in Gracillarioidae (Fletcher et al., 2006) and Drepanidae (Yack et al., 2001; Bowen et al., 2008; Scott et al., 2010; Guedes et al., 2012; Scott and Yack, 2012; Yack et al., 2014; Yadav et al., 2017). 
However, apart from what is known about adults, the sensory mechanisms used by juveniles to engage in these vibratory behaviours remain completely unknown.

In adult insects and arachnids, different types of sensilla are reported or proposed to detect solid-borne vibrations (Yack, 2016), including sensory hairs, scolopidia, slit sensilla, and campaniform sensilla. These sensilla in turn can be grouped and organized in a variety of specialized sensory organs (Yack, 2016). In spiders, vibration reception can be executed by assemblages of cuticular hairs and slit sensilla, the last of which may group to form specialized lyriform organs (Yack, 2016). In adult insects, proposed vibration sensitive sensilla include campaniform sensilla (Kühne, 1982), sensory hairs (Dumpert and Gnatzy, 1977; Kent at al., 1996) and groups of scolopidia organized into chordotonal organs (Yack, 2004; Yack, 2016). Chordotonal organs can be attached to joints and spots on the cuticle, such as in the legs, where they receive vibrations transmitted from the substrate (Young, 1970; Kühne, 1982; Field and Matheson, 1998). They also occur in the antennal Johnston`s organ, where they may have sensitivity to solid-borne vibration (Jeram and Čokl, 1996). The function of chordotonal organs as vibration receptors has been particularly well studied in subgenual organs, which comprises a group of scolopidia in leg tibia that detect vibrations transmitted from the substrate through the leg (Burke, 1954; Howse, 1964; Shaw, 1994b; Kilpinen and Storm, 1997; Yack, 2004). While the types of vibration sensitive organs and their constituent sensilla may differ between taxa, most of these sensory organs share a common feature in that they are in direct or indirect contact with the substrate, usually via the legs (Yack, 2016). 
To date, there is no direct evidence for vibration sensitive organs or sensilla in juvenile insects, particularly in holometabolous insects. Our knowledge about vibration receptors in adults is not directly transferable to juveniles. In these organisms, complex sensory organs such as the Johnston`s organ in the antenna and subgenual organs in the legs do not occur as the morphology of the adult and juveniles are very different. On the other hand, more simple and individualized sensors such as campaniform sensilla have been reported in the mouthparts (e.g. Albert, 1980; Zacharuk and Shields, 1991) and in the thoracic legs (e.g. Kent at al., 1996) while chordotonal organs have been reported throughout the caterpillar body (Lewis and Fullard, 1996; Tamarkin and Levine, 1996; Simon and Trimmer, 2009) and mechano-sensory hairs can be found on many parts of the cuticle (Keil and Steinbrecht, 1984; Keil, 1997; see below). However, none of these sensilla have been studied for possible roles as substrate-borne vibration receptors. In this thesis I will explore the potential role of sensory hairs in detecting substrate-borne vibration, since caterpillars have hairs that touch the substrate, and since it has already been shown that some hairs in caterpillars detect vibration from other media such as the air.

Sensory hairs occur on many parts of the body wall in caterpillars (Stehr, 1987), but the functions of these receptors are generally poorly understood. Based on a series of studies conducted on one caterpillar species, Barathra brassicae, it is well known that some sensory hairs project from the caterpillar's body into the air to detect near field sounds of predatory or parasitic flies and wasps (Markl and Tautz, 1975; Tautz, 1977; Tautz, 1978; Tautz and Markl, 1978). But to date there is no direct evidence that they are used for detecting solid-borne vibrations from the substrate. Cuticular hairs occur on the 
prolegs of many caterpillar species (Stehr, 1987) but the functions have not been reported. At least one caterpillar species, Manduca sexta, is known to have hairs that point down and touch the substrate, but the current hypothesis for the role that some of these may have, is to increase the physical range of tactile perception in the prolegs, thus gathering anticipated information about the terrain for fine tuning of crawling (van Griethuijsen and Trimmer, 2010). Thus, hairs on the prolegs might have functions that remain undescribed.

From a mechano-sensory perspective, vibration receptors in insects can produce neural responses that may aid characterizing different parameters of mechanical waves. For instance, some sensory hairs can produce action potentials at specific phases of the stimulus (Tautz, 1978; Shimozawa and Kanou, 1984) and present directional sensitivity (Tautz, 1978; Tautz and Markl, 1978; Shimozawa and Kanou, 1984). The characterization of stimulus amplitude by mechanoreceptors may be aided by the magnitude of neural response, i.e. rate of action potentials (Eberhard et al., 2010). In addition, mechanoreceptors, including sensory hairs, can respond independently to one out of three kinematic quantities of vibration: Displacement (Tautz, 1978), velocity (Shimozawa and Kanou, 1984) and acceleration (Shimozawa and Kanou, 1984). These are relevant features for transducing different characteristics of mechanical waves and may also occur in other putative vibration receptors that remain to be described.

In this study I tested the hypothesis that proleg hairs detect vibratory stimuli, using the cabbage looper, Trichoplusia ni, as a model. I chose this species as they are readily available throughout the year from a provincial supplier (see Methods) and importantly, they possess hairs on the prolegs that are in contact with the plant substrate. 
While at present there is no direct evidence that this species responds to vibration, it is assumed that some form of vibration reception to detect predators or physical elements is necessary for most plant-bound larvae (Bacher et al., 1996; Guedes et al., 2012; Yack, 2016). Specific goals were to confirm if hairs on the prolegs of these caterpillars are sensory and whether they respond to vibrations. If so, the goals extended to answer the following questions: 1) What is the sensitivity threshold across frequencies? 2) Does the response increase with stimulus amplitude? 3) Does the hair have directional sensitivity? 4) Is the response rapid or slow adapting (phasic or tonic)? 5) Does the hair present phase locking? 


\section{Materials and Methods}

\section{Animals}

T. $n i$ caterpillars were purchased from Great Lakes Forestry Centre (Sault Ste. Marie, Canada) as young instars in plastic cups with artificial diet and reared in it as they aged. Caterpillars were kept under varying temperatures, from $18^{\circ} \mathrm{C}$ to $28^{\circ} \mathrm{C}$, to allow different development times and optimize the use of the acquired batches. Some were put in the fridge for a few days to slow down development. As I did not track moulting of individual caterpillars, their instar was estimated using size values available in the literature (Jones et al., 1981) and caterpillars of the $4^{\text {th }}$ instar were used in the neurophysiological experiments described below. Neurophysiological experiments were conducted on more than 30 caterpillars, but results presented here report on twelve individuals whose experiments were successful.

\section{External Morphology}

For identifying which proleg hairs touch the substrate, $T$. ni caterpillars were kept on tomato leaves in resting position and visualized under a stereomicroscope (M205C, Leica Microsystems GmbH, Wetzlar, Germany). Photographs were taken using Leica software (LAS4.3, Leica Microsystems $\mathrm{GmbH}$ ) and a camera attached to the microscope (DMC4500, Leica Microsystems GmbH). The hair located most laterally on the proleg was chosen for the neurophysiological experiments due to its accessible position and the confirmation that it contacts the substrate when the caterpillar is in resting position. The location of this hair facilitated the experiment because the arrangement of the stimulation-recording set-up described below (Figure 2.1) was impaired in the others. 
For instance, in the other hairs the steel electrodes and the forceps would hardly be positioned without touching each other, due to the proximity between the Ventral Nerves and the hairs. Presumably, all hairs would have similar responses due to their similar morphology. My study focuses on one of them only.

\section{Neurophysiological recordings}

\section{Specimen preparation}

To record from the ventral nerve, I followed a modified protocol of Peterson and Weeks (1988). Live caterpillars were anesthetized with carbon dioxide for less than one minute. Using a cotton thread, the abdomen-thorax junction was ligated with a surgeon's knot to cease hemolymph flow between them. The thorax was cut off posteriorly to the knot and discarded to reduce neural activity and movements in the abdomen. The tied abdomen was pinned to a piece of modeling clay keeping the ventral surface of the insect directed upwards. Using microdissection scissors, an access hole was opened in the ventral surface of abdominal cuticle in segment A5 to expose its neural ganglion. Left and right Ventral Nerves, which contain the afferent neurons innervating prolegs in the respective segment, were identified based on Eaton (1988) and Peterson and Weeks (1988). A modified saline from Platt and Reynolds (1986) was used, which contained the following ingredients: 1 pouch of buffer (P3813, Sigma-Audrich, Saint Louis, USA) diluted in $750 \mathrm{~mL}$ of distilled water, $\mathrm{CaCl}_{2} 1.56 \mathrm{mM}$ and sucrose $102 \mathrm{mM}$. The dissection procedure is demonstrated in one supplementary video in the appendix for a Drepana arcuata caterpillar (Appendix D.1 - Short tutorial for exposing a caterpillar's nervous ganglion). 


\section{Extracellular Nerve Recordings}

Extracellular hook electrode recordings were conducted on the ventral nerve of A5 ( $5^{\text {th }}$ abdominal segment) in most caterpillars, although in caterpillars \#3 and \#4 the recordings were made on A6. Similar results were expected across prolegs since the anatomy of the hairs is similar and nervous afferents were shown to be the same in other species (e.g. Peterson and Weeks, 1988). Microelectrodes were manufactured from stainless steel rods (712700, AM Systems, Sequim, USA) using a function generator (SG 1274 Heath Company, USA) to electrolytically etch them in 4\% hydrochloric acid solution against a graphite rod and using a rosin activated tin-lead wire solder (Solder SN60/Pb40 RA 3\% .032 DIA, AIM, Montreal, Canada) to solder them to male connector pins (521200, AM Systems). The sharpened tip of the steel electrode was bent and hooked onto the root of the Ventral Nerve ipsilateral to the tested proleg. A second steel electrode used as a reference was immersed in the hemolymph farther away from the nervous system, typically within the same access hole that had been opened on the cuticle. The electrodes were connected to the head stage of a Differential Amplifier (Model 3000, A-M Systems, Sequim, USA) and the neural signal was recorded into a digital processor (TDT RP 2.1, Tucker-Davis Technologies, Gainesville) (see details below). An example of baseline neural activity for Drepana arcuata is shown in one supplementary video in the Appendix (Appendix D.2 - Neural activity recorded from ventral nerve). To confirm sensory activity, in some specimens (see Results) the neural recordings were conducted with the Ventral Nerve de-efferented by cutting the nerve proximally from the recording electrode. 


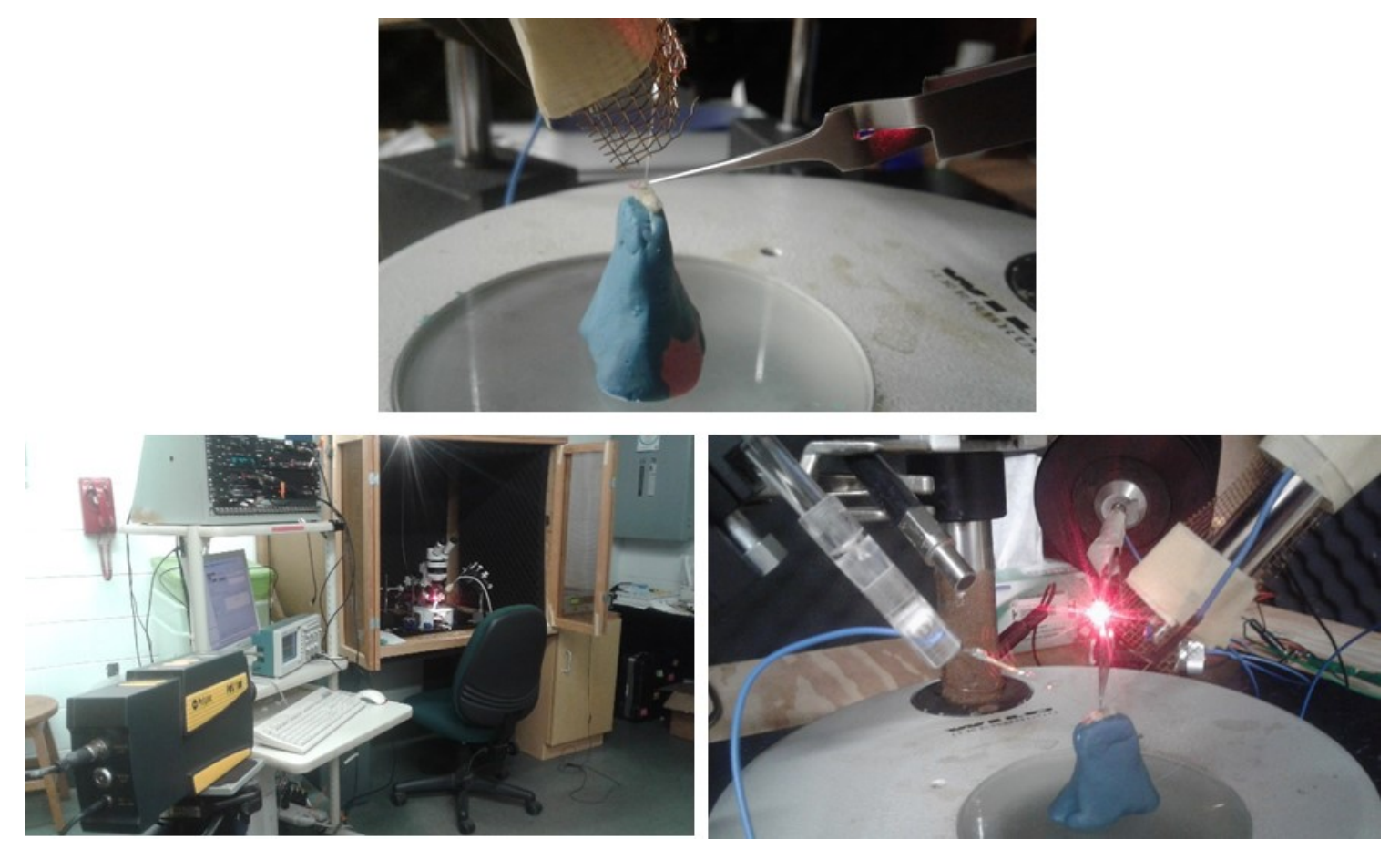

Figure 2.1 - Neurophysiology experimental set-up. On top, forceps are shown touching the caterpillar, which is pinned to a piece of modelling clay. While the forceps hold the proleg hair, a Doppler laser vibrometer (bottom left) records the mechanical stimuli applied in real time by measuring the movements of a mirrored tape attached to the forceps (bottom right). The bottom right photo also reveals the mini-shaker to which the forceps were attached. 


\section{Stimulus application and calibration}

To apply vibration stimuli directly to the proleg seta, I used self-closing stainless-steel forceps with a tip width of approximately $100 \mu \mathrm{m}$ (JBS, Switzerland) attached to the vibrating plate of a Mini-shaker (Type 4810, Brüel \& Kjaer, Nærum, Denmark) with modeling clay which in turn was secured with adhesive tape (Figure 2.1). The Minishaker was supported on micromanipulators and powered by an amplifier (Type 2718, Brüel \& Kjaer, Nærum, Denmark). Using this set-up, the Mini-shaker could be gently moved to a position where the forceps could grip and stimulate the most lateral subventral hairs in the proleg. Two short supplementary videos in the appendix show the forceps being attached in two orthogonal directions (Appendix D.5 - Gripping hair at antero-posterior direction and Appendix D.6 - Gripping hair at latero-medial direction). Calibration of the applied vibrations was done prior to the experiment in stimulation trials using a Laser vibrometer (PVD-100, Polytec Inc., Germany) pointed onto a piece of mirrored tape attached to the forceps. In this process, peak voltages of the Laser output were measured on an oscilloscope (TDS2012C, Tektronix, Beaverton, EUA) and converted to peak velocities using conversion factors provided by manufacturer. The analog knobs of the amplifier powering the Mini-shaker were adjusted so that applied vibrations achieved pre-calculated amplitudes. The positions of the knobs for each frequency were annotated on the amplifier interface for further resetting during the experiment. To keep accurate measurements of vibrational stimuli during the experiment, the output from the Laser vibrometer was recorded into the second channel of the digital processor (TDT RP 2.1) simultaneously with the neural response. As it can be noticed on Figure 2.1, the Laser Vibrometer was not pointed perfectly perpendicular 
to the vibrating plate of the mini-shaker, i.e. there was an angle between the direction of the motion and the direction of the laser. Presumably, this caused the measured velocity to be smaller than the true velocity. The effect of the angle deviation on measured amplitudes by the laser was corrected using empirical factors determined in preliminary experiments and confirmed by trigonometric calculations - true velocity was obtained from the division of measured velocity by 0.92 . This value corresponds to $\cos 23^{\circ}$, which was the approximate angle between the laser and the direction of motion.

\section{Stimulus Presentation}

Two types of stimuli were used: Sine and Step. Sine stimuli consisted of pure tone sinusoidal pulses of $250 \mathrm{~ms}$ duration, including $25 \mathrm{~ms}$ rising and decaying phases. Frequencies ranged from 10 to $905 \mathrm{~Hz}$ and peak-to-peak displacement amplitudes from 0.02 to $64 \mu \mathrm{m}$. An example of a sine stimulus is shown in Figure 2.2, which contains a sine signal displayed in all three proxies of amplitude, i.e. displacement, velocity and acceleration. Sine stimuli are also demonstrated in two supplementary videos in the Appendix (Appendix D.7 - $10 \mathrm{~Hz}$ sine vibration and Appendix D.8 - $80 \mathrm{~Hz}$ sine vibration). Step stimuli consisted of a fast motion impulse that displaced the hair followed by a slow return to its resting position. Peak-to-zero displacement amplitudes ranged from 0.02 to $23 \mu \mathrm{m}$. An example of a step stimulus is shown in Figure 2.3, also containing the three proxies of amplitude. Step stimuli are also demonstrated in one supplementary video in the Appendix (Appendix D.9 - Step stimulus (single pull/push)). Signals were programmed in PC Tucker Davis software (RpvdsEx, v. 5.4; Alachua, USA) and generated by a digital signal processor (RX6 multifunction processor, Tucker Davis Technologies). 

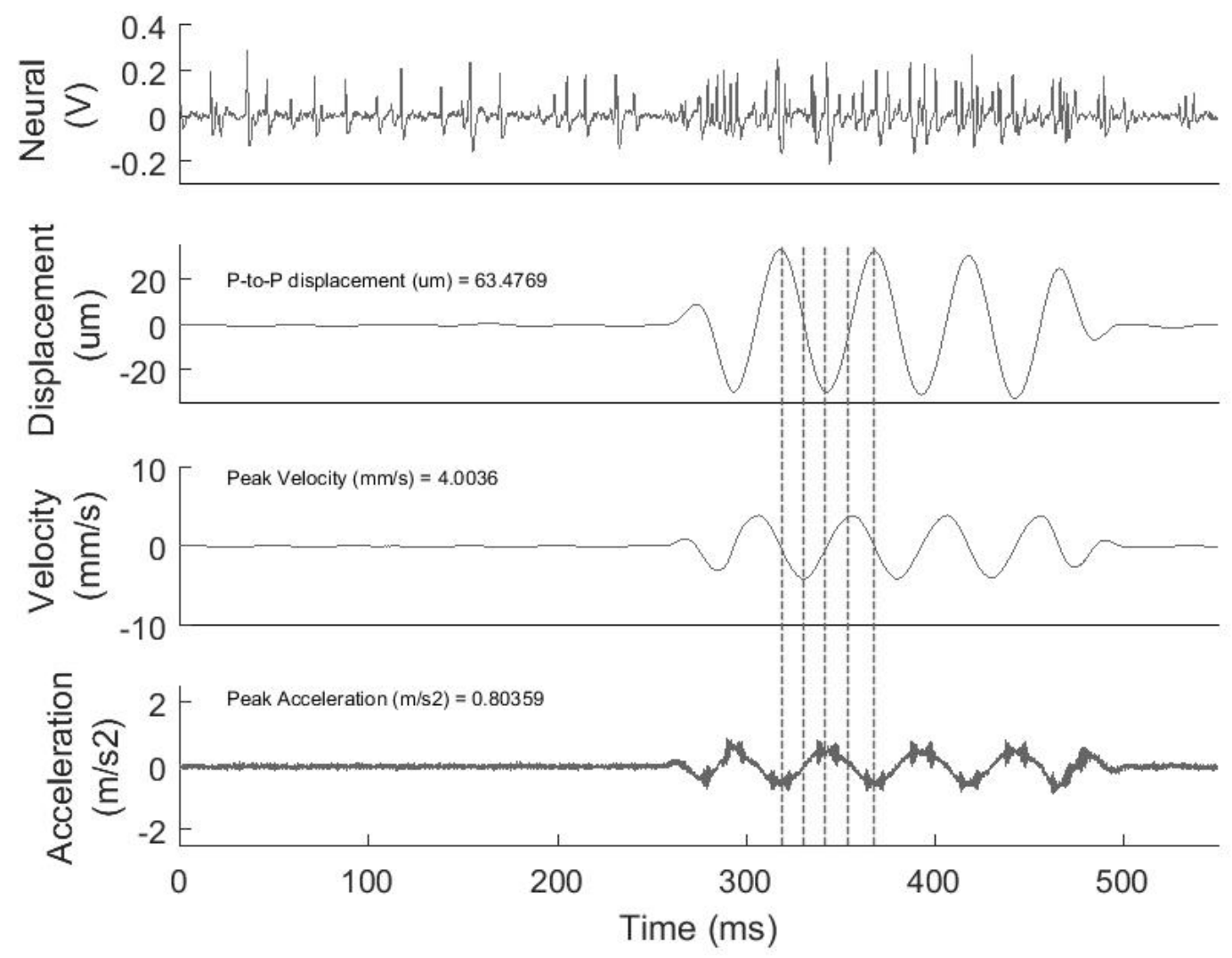

Figure 2.2 - An example of sine vibrational signal as measured by the laser vibrometer after filtering and converted to different proxies of vibration amplitude. The signal has a frequency of $20 \mathrm{~Hz}$ and was applied to caterpillar \#8, whose neural response is shown on the top trace. Dashed lines are shown to facilitate identification of shifts between phases of different proxies. For instance, Displacement is shifted $90^{\circ}$ relative to Velocity, which in turn is shifted $90^{\circ}$ to Acceleration. A trace of neural response is shown on the top window, which will be later explained in the Results. See supplementary videos in the Appendix for a better understanding (Appendix D.7 - $10 \mathrm{~Hz}$ sine vibration; Appendix D.8 - $80 \mathrm{~Hz}$ sine vibration). 

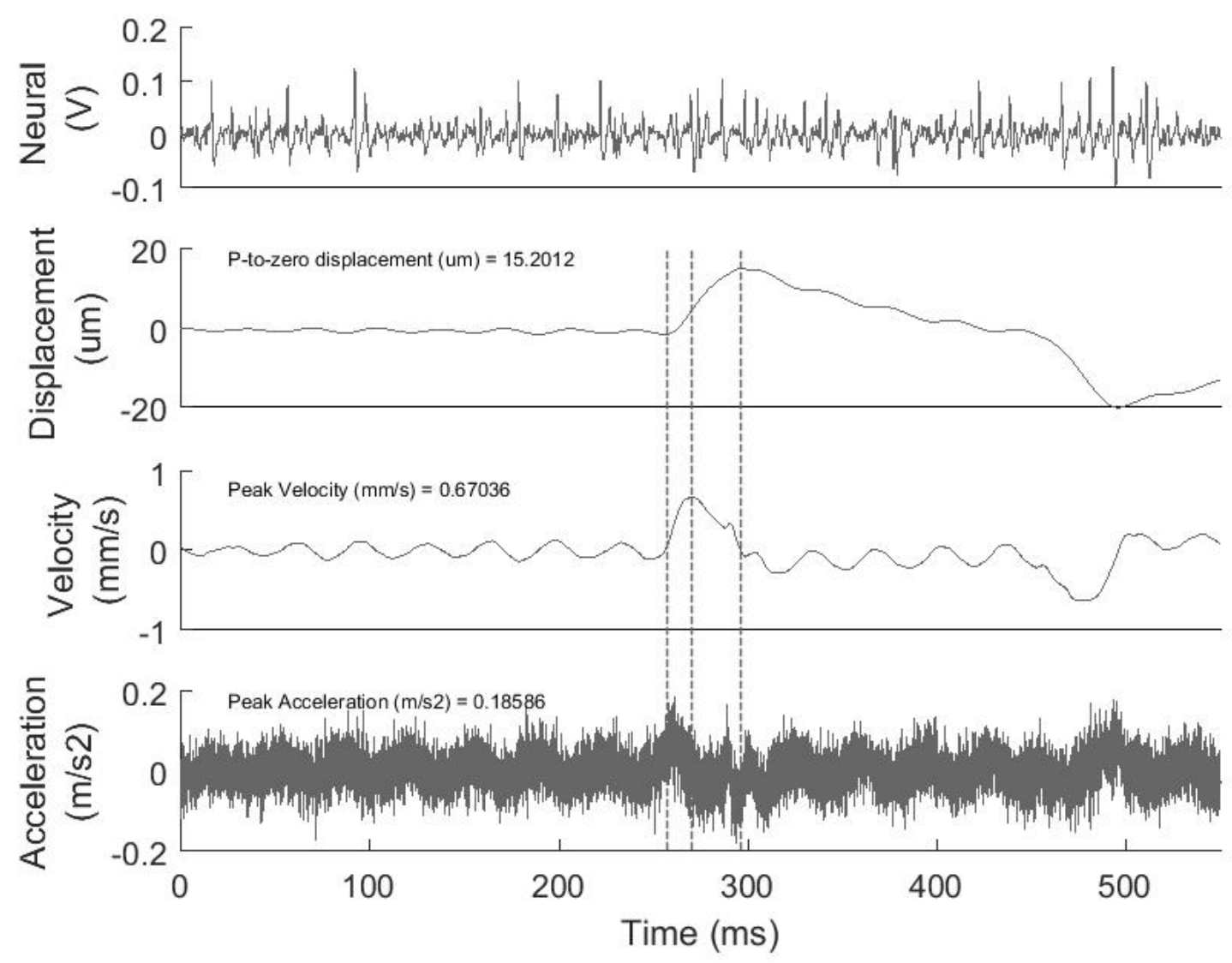

Figure 2.3 - An example of step stimulus calculated in the three proxies of amplitude vibration. This signal was applied to caterpillar \#19 in the latero-medial direction. Whereas the first stroke, indicated by dashed lines, is pushing the seta in the medial orientation (towards the proleg), the second stroke, visible at $\sim 480 \mathrm{~ms}$, is pulling the seta laterally (away from the proleg). The amplitude of this signal in the velocity proxy is 0.67 $\mathrm{mm} / \mathrm{s}$, which corresponds to the threshold, as it will be described later in the Results section. A trace of neural response is shown on the top window, which will be later explained in the Results. See the supplementary video in the Appendix for a better understanding (Appendix D.9 - Step stimulus (single pull/push)). 


\section{Directionality}

Both types of stimuli described above were applied in multiple directions. This was done by rotating the modeling clay holding the specimen and moving the Mini-shaker with the forceps around it. Sine stimuli were applied in two orthogonal axes relative to the caterpillar: antero-posterior and lateral-medial. Step stimuli were applied at the same

orthogonal axes, but either pushing or pulling the seta, resulting in movements in four directions: anterior, posterior, lateral and medial. Figure 2.4 shows two photos of the experiment in which the hair was stimulated in different orthogonal axes. 

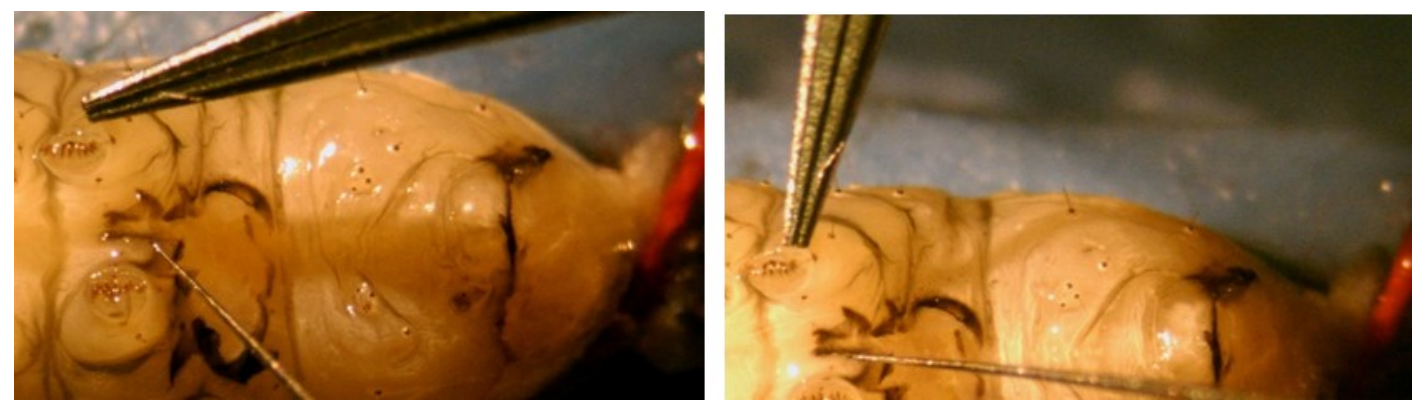

Figure 2.4 - Ventral view of a $T$. ni caterpillar abdomen for directional experiment. The forceps are holding the sensory hair of the right proleg on segment A5 at two different orthogonal axes: antero-posterior (left) and latero-medial (right). In both photos, the stainless-steel recording electrode is seen diagonally on the bottom and is inserted into the access hole to hook on the Ventral Nerve. A red thread is visible on the right, which is ligating the most anterior part of the abdomen. The stimulated hair, which is located laterally on the proleg, is not visible because it is hidden by the forceps. A demonstration of how the hair was gripped by the forceps in this caterpillar is shown on supplementary videos in the Appendix (Appendix D.5 - Gripping hair at antero-posterior direction and Appendix D.6 - Gripping hair at latero-medial direction). 
Measured variables

Using MatLab R2016b (MathWorks Inc., Natick, MA, USA), all proxies of amplitudes, i.e. displacement, velocity and acceleration, could be calculated for the stimuli (Figure 2.2 and Figure 2.3). Also in Matlab, an algorithm was used to detect and count individual action potentials. An example trace with detected spikes is shown in Figure 2.5. For the phase locking analysis, the phases at which each spike occurred, relative to the stimulus waves, were also detected in Matlab and expressed as angles. Figure 2.6 illustrates and gives an example of how phase was determined. The type, frequency, amplitude and direction of all stimuli were exported to .csv files along with number of action potentials occurring during and before each signal and spike relative phases for further analysis in R Statistical Software (R Core Team, 2016). Sample of data exported for the threshold and magnitude analysis, the directional analysis and the phase locking analysis are shown in the Appendix (Appendix A.1 - Tables with samples of data). 

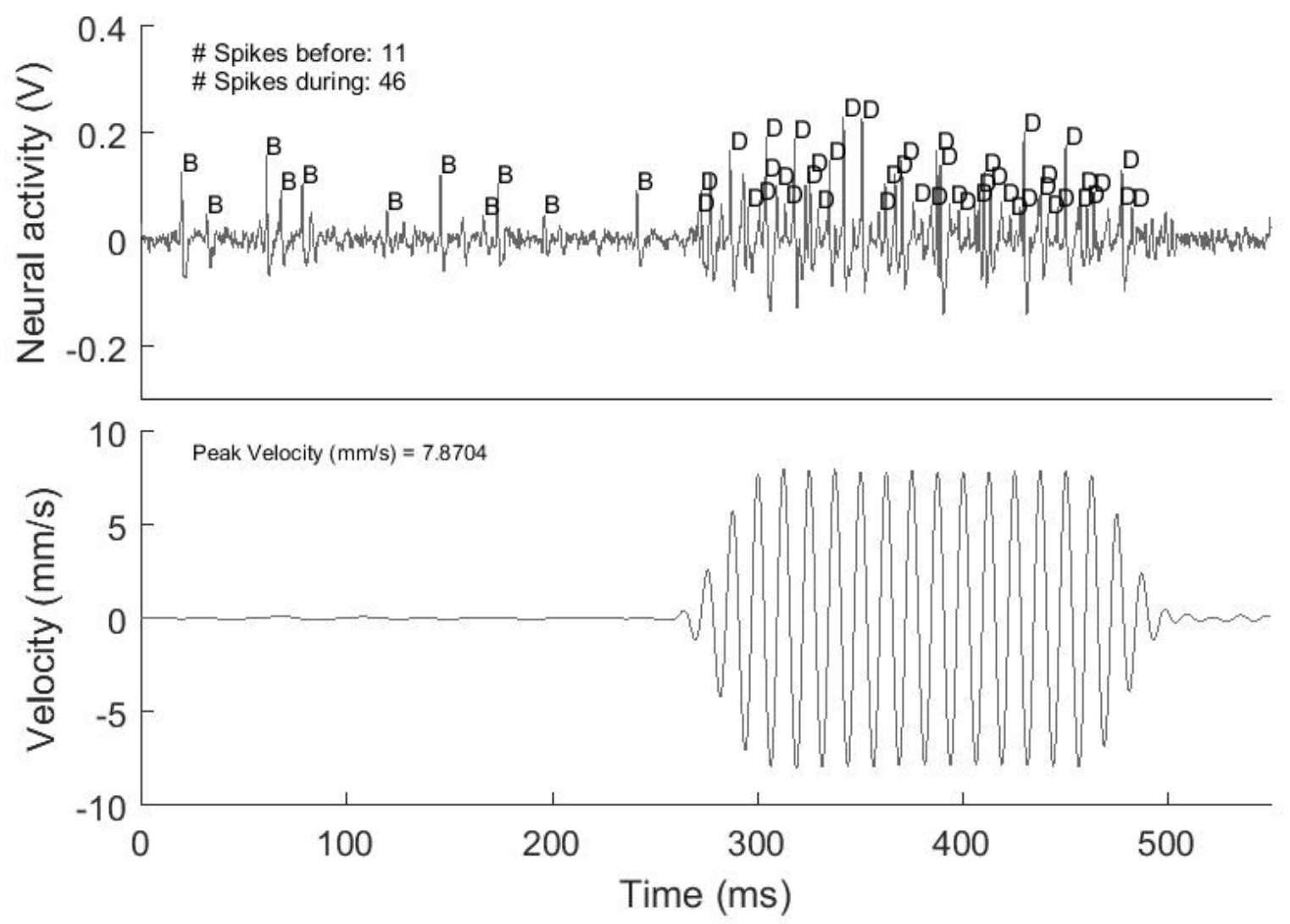

Figure 2.5 - An example trace showing detected spikes. Spikes occurring before and during the stimulus are indicated by letters B and D, respectively. The stimulus signal was recorded from caterpillar $\# 8$ and has a frequency of $80 \mathrm{~Hz}$. Information on number of occurring spikes during neural response will be given in the Results section. 

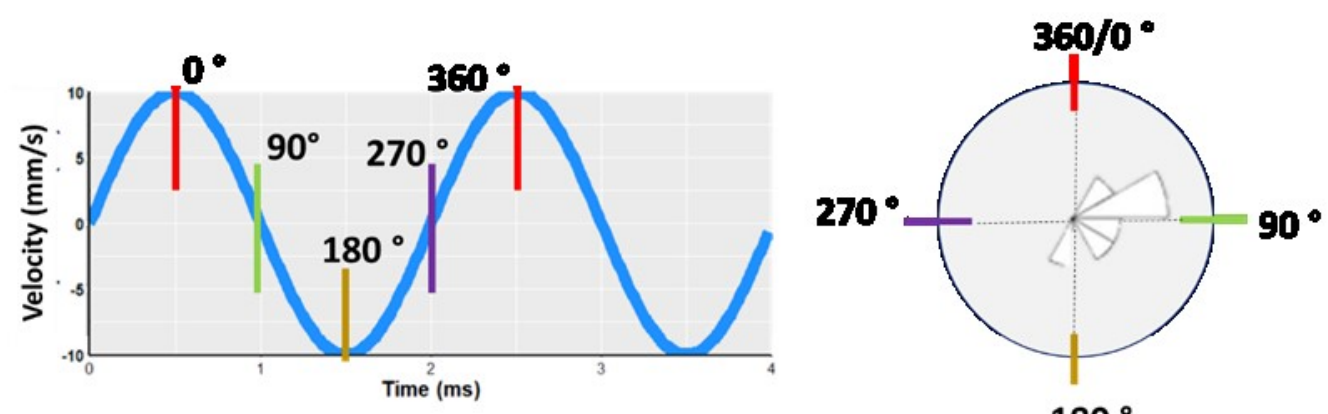

$180^{\circ}$

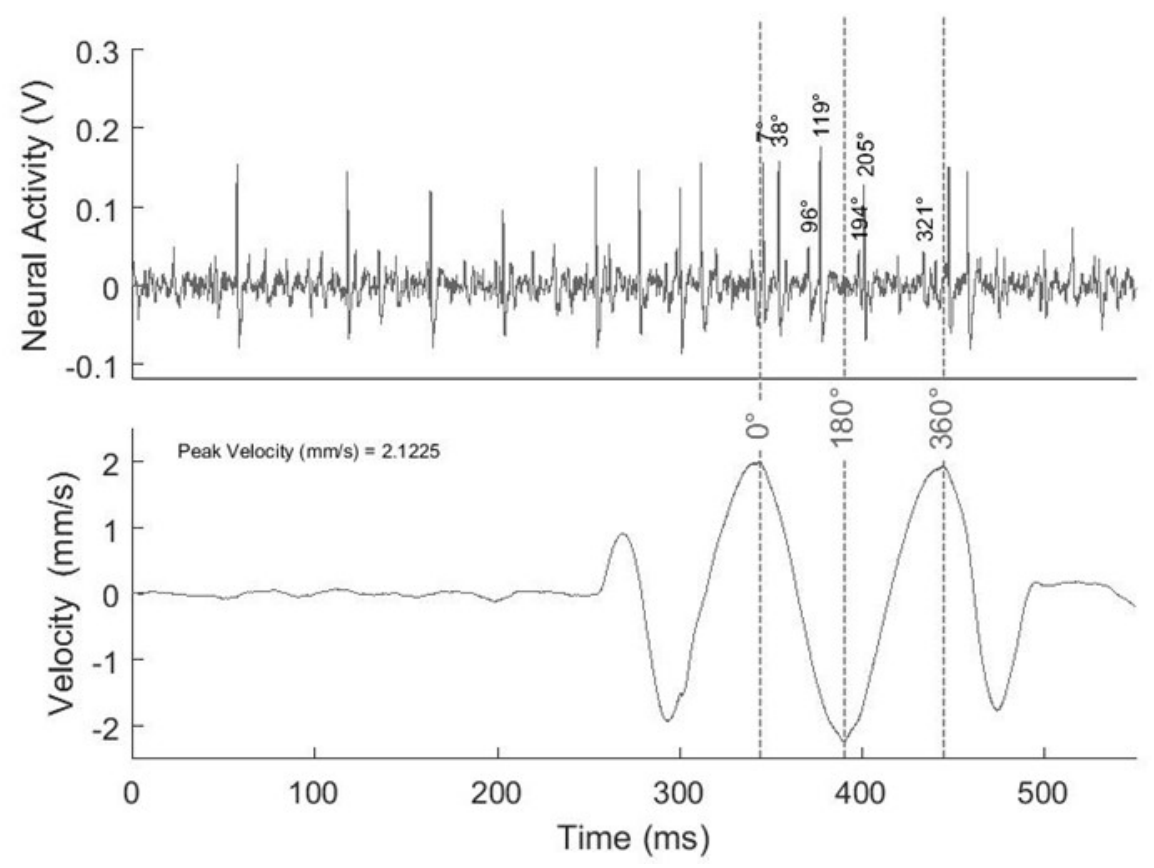

Figure 2.6 - Angles assigned to spikes for phase locking analysis. On top, a diagram is illustrating how angle values can be assigned to spikes based on the phase of the stimulus at which they occur. It is also showing how these angles can be represented in a rose histogram. On bottom, a practical example obtained from caterpillar \#8 is showing what angles would be assigned to spikes occurring during a wave cycle of a $10 \mathrm{~Hz}$ sine stimulus signal. Dashed lines indicate the intervals used by the algorithm to assign angles for this particular wave. More information on stimulus phases and spike phase-locking will be given in the Results section. 


\section{Statistical analyses}

\section{Thresholds}

Local regression analysis (loess(), R Core Team, 2016) was executed individually on every frequency for each caterpillar, inputting stimulus amplitude (i.e. displacement, velocity or acceleration) as the independent variable and difference in number of spikes between during and before a stimulus as the dependent variable. Point wise 99\% confidence intervals were calculated by multiplying the standard errors of the fit by 0.995 lower and upper quantiles of a t-distribution, with the number of degrees of freedom corresponding to that of the local regression ( $q$ t(), R Core Team, 2016). A statistical significant threshold was defined as the lowest velocity at which the lower confidence interval achieved a difference in number of spikes that was larger than zero. The adjustment of the local regressions to the data was verified by plotting raw data points with their respective regression function, 99\% confidence intervals and $95 \%$ prediction intervals (loess.sd(), Scrucca, 2011)(Figure 2.7). All plots generated, with respective threshold values, are provided in the Appendix (Appendix A.2 - Local regressions loess() for all frequencies in all $T$. $n$ i caterpillars). 

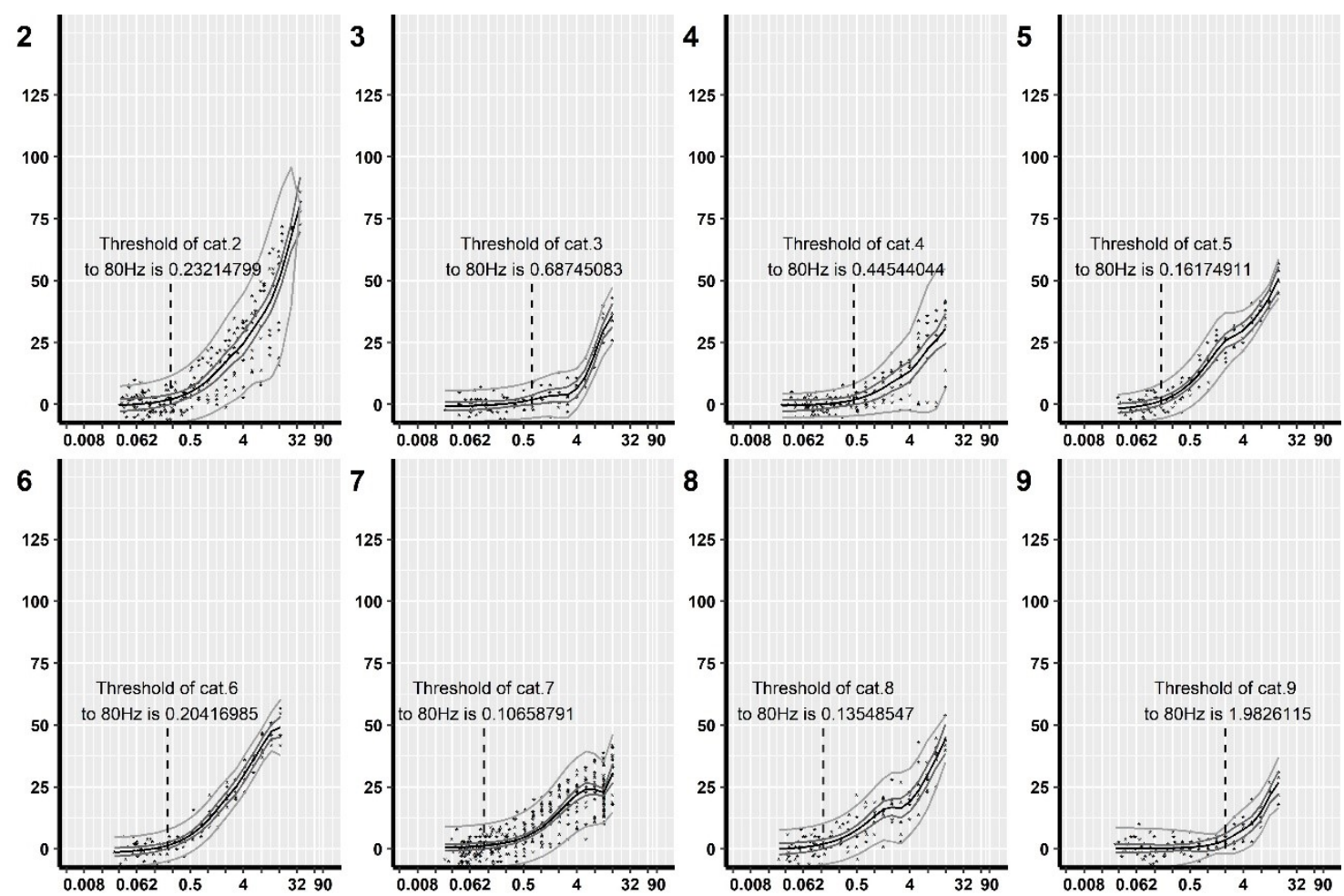

Figure 2.7 - Local regressions loess() for $80 \mathrm{~Hz}$ frequency in all T. ni caterpillars. Each subplot shows data from one caterpillar, whose ID is indicated by the number label on its top left. Units on the horizontal axes are Peak velocity $(\mathrm{mm} / \mathrm{s})$ and on the vertical axes are difference in \# spikes (i.e. \# spikes during minus \# spikes before a stimulus). Each data point represents one stimulus signal. Black, dark grey and light grey lines represent the fit, 99\% confidence intervals and $95 \%$ predict intervals of the local regression, respectively. The dashed line at each subplot indicates the point at which the lower confidence interval surpassed the zero line for the first time, which was defined as the threshold for that specific caterpillar to that frequency. Loess regressions for the other frequencies are shown in the Appendix (Appendix A.2 - Local regressions loess() for all frequencies in all $T$. $n i$ caterpillars). 


\section{Sensitivity curve}

To test if thresholds differ significantly across frequencies, three Kruskal-Wallis tests were executed (kruskal), de Mendiburu, 2016), one for each proxy of stimulus amplitude. Frequency and thresholds were inputted respectively as independent and dependent variables and each caterpillar consisted of a replicate.

\section{Magnitude of response}

Local regressions indicated that magnitude of response increased with stimulus amplitude, but to determine whether it varies across frequencies, the number of spikes detected during vibrational signals at a supra-threshold velocity that was common for all frequencies was averaged for each caterpillar and compared using a One-way Anova with Welch correction (oneway.test(), R Core Team, 2016).

\section{Directionality}

To test the effect of different stimulus orientations on the sensitivity of the seta, the difference in number of spikes before and during stimulus signals with a supra-threshold velocity were compared using a Welch t-test (t.test), R Core Team, 2016) for the two

orientations of the $80 \mathrm{~Hz}$ frequency stimulus and an One-way ANOVA (aov), R Core Team, 2016) followed by a Tukey HSD test (HSD.test(), de Mendiburu, 2016) for the four orientations of the step stimulus. 
Rapid or slow adapting response (phasic or tonic)?

To determine whether the sensory hair has a rapid or slow adapting response to the stimulus, the number of action potentials happening during different intervals of the step stimuli were compared. Four intervals with equal durations of $50 \mathrm{~ms}$ were determined, one occurring before and the other three occurring during step stimuli. This duration was chosen because it corresponds to the slope of the stimulus in the displacement proxy, i.e. when the hair is being moved by the forceps under the highest velocity. Just suprathreshold stimuli, above $0.5 \mathrm{~mm} / \mathrm{s}$, were included in the analysis. The four intervals were compared using a Kruskal-Wallis test (kruskal), de Mendiburu, 2016). 


\section{Results}

\section{External Morphology}

Three hairs are found close to the proleg planta, which is the basal part that holds onto the substrate though gripping crochets. The hairs are located anteriorly, medially and laterally, respectively. They attach to projections of the cuticle, where they have an approximate diameter of $30 \mu \mathrm{m}$. Their total length is approximate $600 \mu \mathrm{m}$. Visualization of the medially located hair was impaired by the caterpillar position, but the other two hairs were found to keep an intimate contact with the substrate (Figure 2.8). 

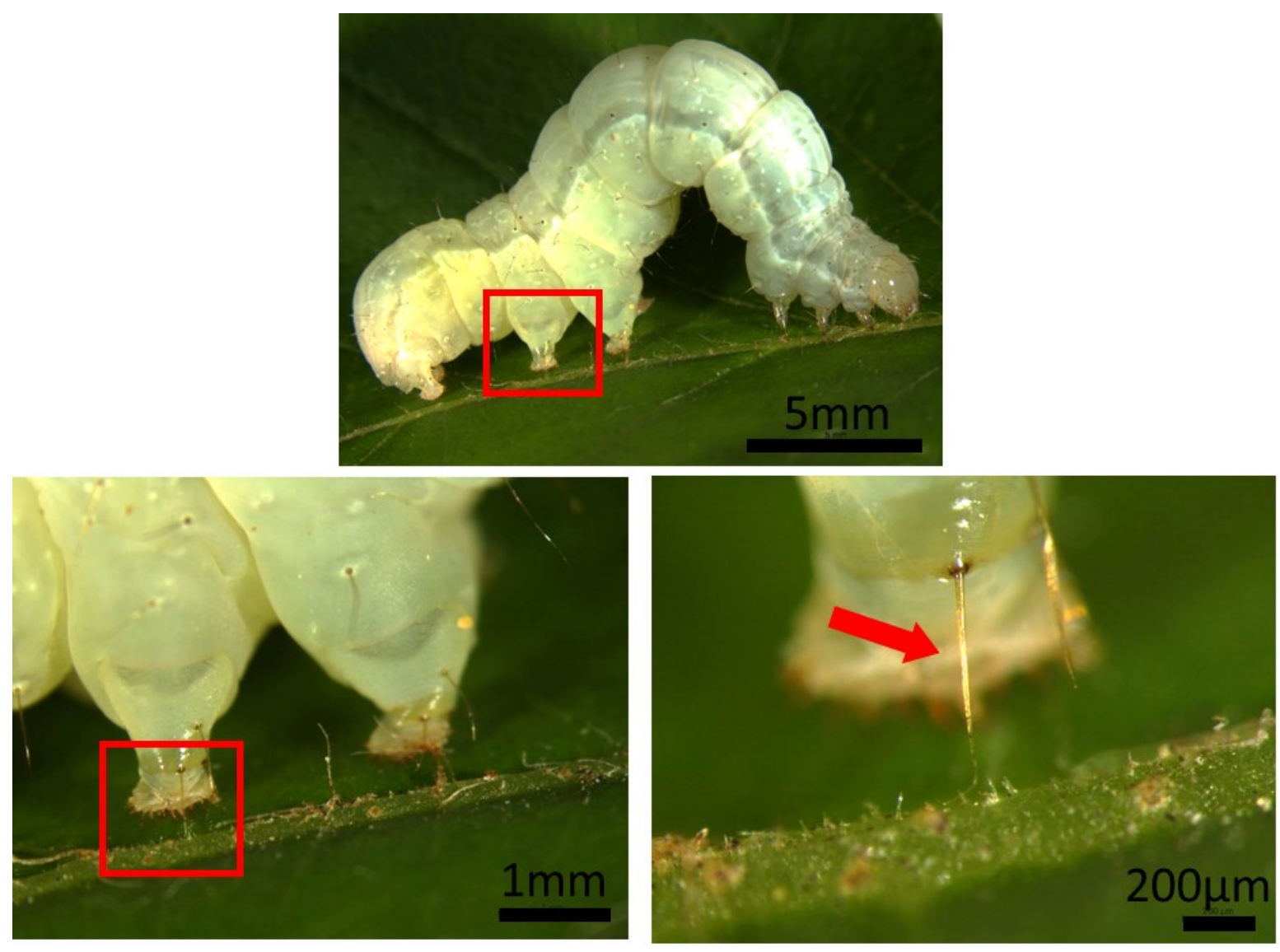

Figure $2.8-T . n i$ caterpillar resting on a leaf (top), with the proleg holding on the leaf (bottom left) and the lateral hairs in close contact with the substrate (bottom right). Red arrow indicates the most lateral hair that was explored in the neurophysiological experiments of this study. 


\section{Thresholds and sensitivity curve}

First, is the neural activity observed during stimuli a consequence of sensory input or an artifact from motor activity? Since de-efferenting the nerve did not affect the occurrence of neural response (Figure 2.9), the response must have a sensory origin. Moreover, deefferented animals showed a similar response throughout all tested frequencies and amplitudes (Appendix A.3 - Sample traces of neural activity from proleg hair in $\boldsymbol{T}$. ni), confirming that affirmation. Second, what are the thresholds at different frequencies? The range of amplitudes that I applied was enough to record neural activity at subthreshold, at threshold and at supra-threshold (Figure 2.10). Moreover, this could be done for all frequencies and neural traces happening in response to stimuli of different intensities were recorded (Appendix A.3 - Sample traces of neural activity from proleg hair in $\boldsymbol{T}$. $\boldsymbol{n i})$. The thresholds do not differ in the velocity proxy $\left(\mathrm{H}_{7}=10.40\right.$, $\mathrm{p}=0.1671$ ) and have a mean $\pm \mathrm{SD}$ of $0.75 \pm 0.83$ (Figure 2.11). In the displacement and acceleration proxy thresholds are significantly different across frequencies (displacement: $\mathrm{H}_{7}=47.20, \mathrm{p}<0.0001$; acceleration: $\left.\mathrm{H}_{7}=25.27, \mathrm{p}=0.0007\right)$. 

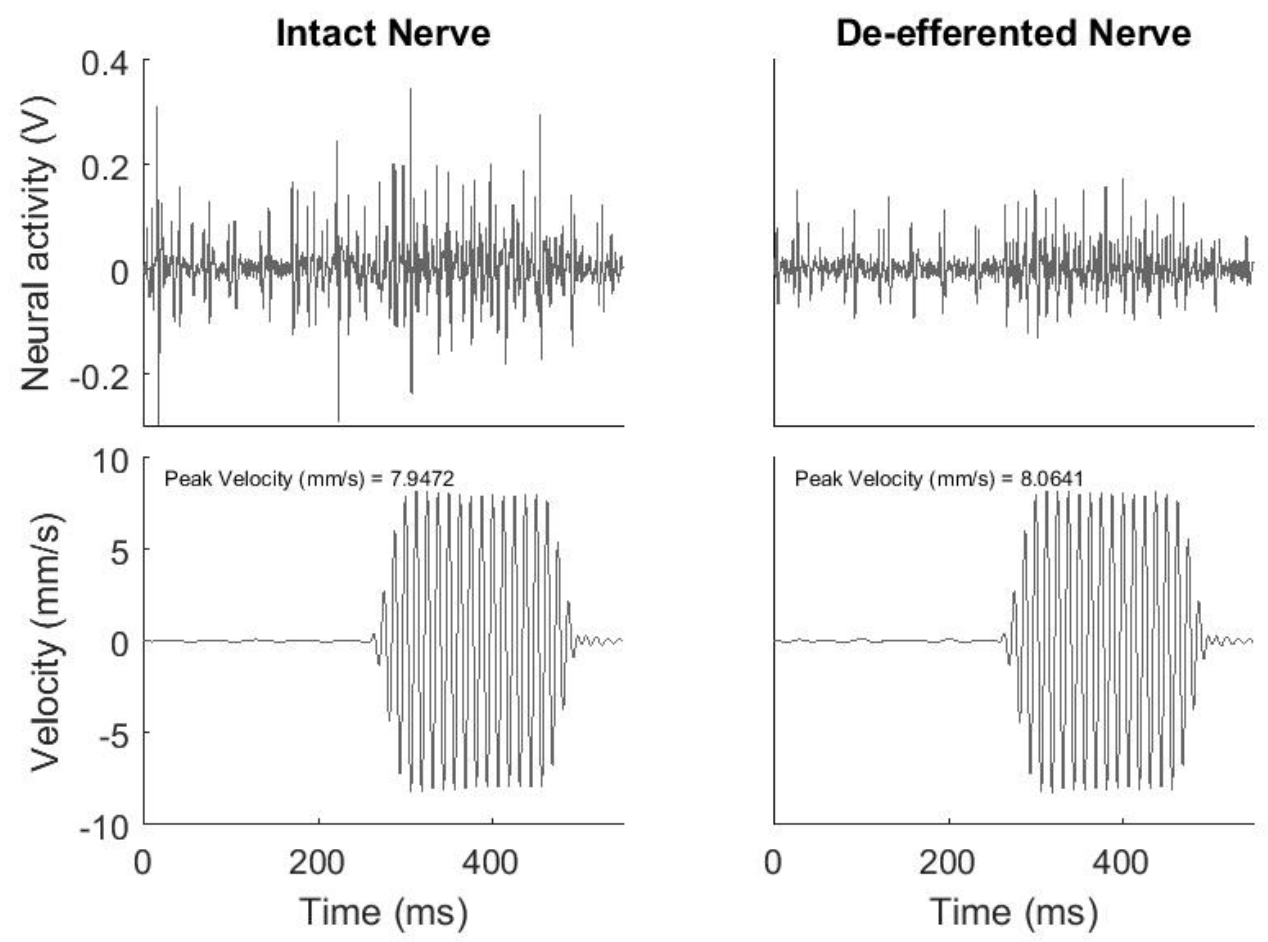

Figure 2.9 - Example of traces recorded from a de-efferented nerve. Traces were recorded immediately before and after de-efferenting the Ventral Nerve of caterpillar \#7. The neural response obtained when the sine vibrational stimulus was applied to the hair continued to occur even after de-efferenting, confirming that the response is sensory. An expanded set of sensory responses from a de-efferented caterpillar is shown in the Appendix (Appendix A.3 - Sample traces of neural activity from proleg hair in $T$. $n i$ ). 

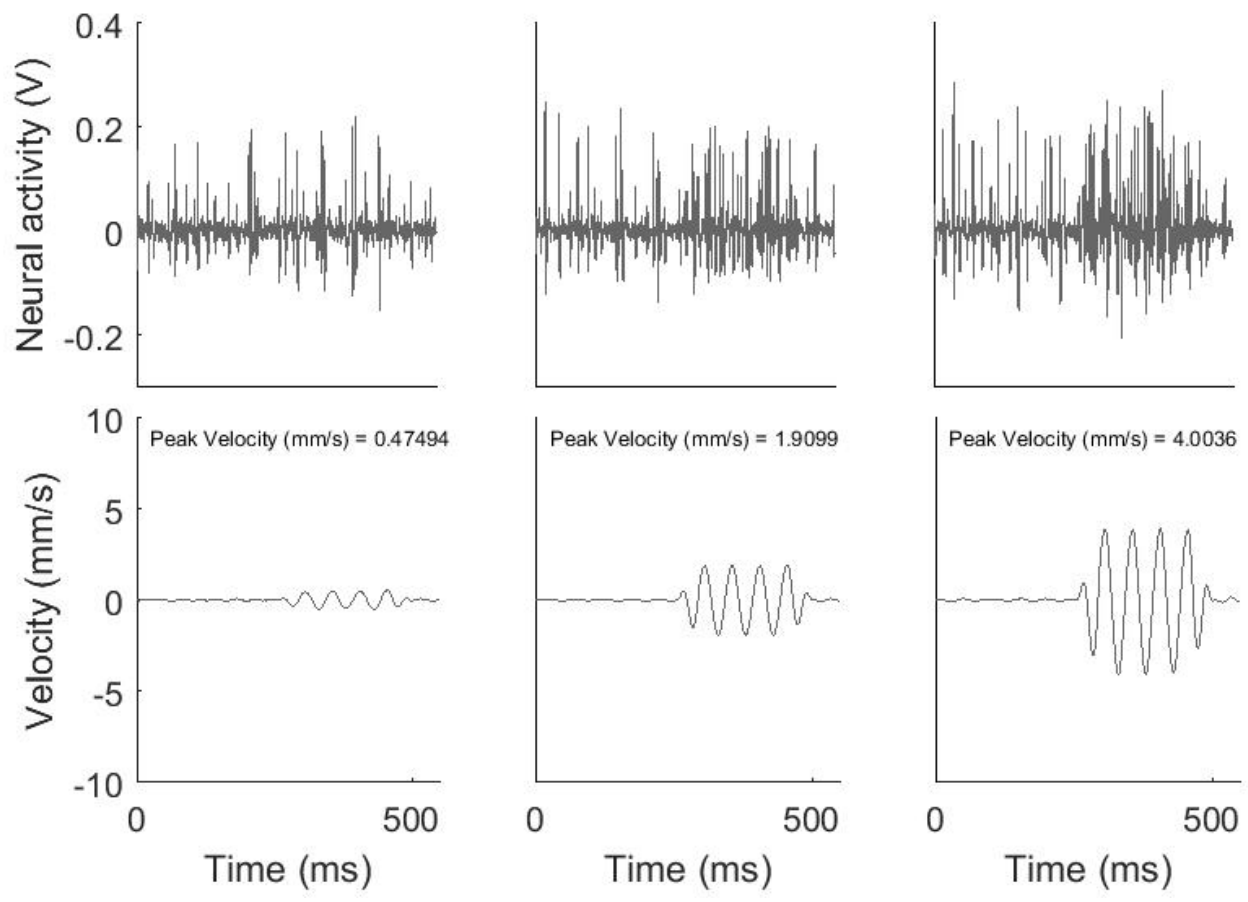

Figure 2.10 - Sensory responses to stimuli of different intensities in $T$. ni. The windows on the bottom show the stimulus applied as recorded by the laser, in the velocity proxies. The windows on top show respective neural responses. The peak velocity for each stimulus is shown on the bottom windows. 


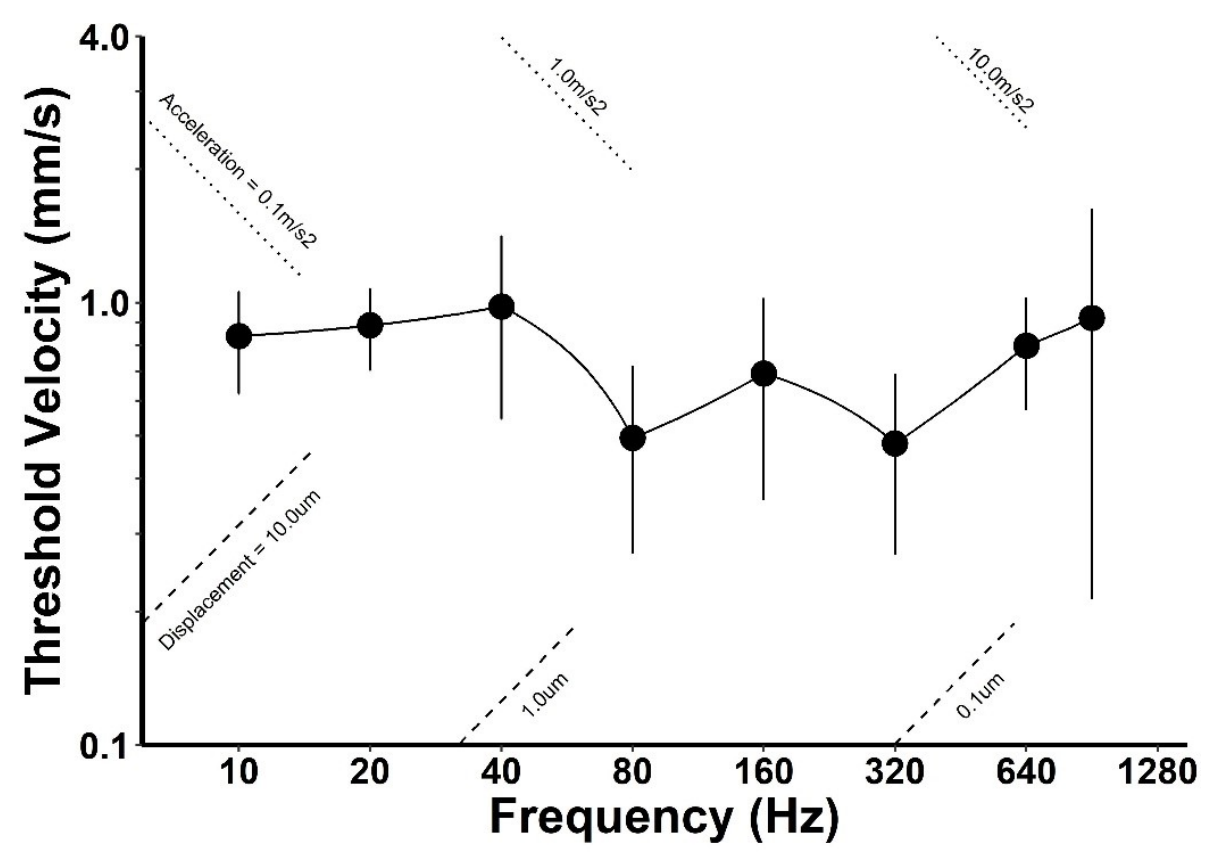

Figure 2.11 - Average thresholds to different frequencies on the velocity proxy do not differ significantly $\left(\mathrm{H}_{7}=10.40, \mathrm{p}=0.1671\right)$, which means the hair is likely a velocity receptor. Frequencies from 10 to $640 \mathrm{~Hz}$ are represented by 8 animals whereas $905 \mathrm{~Hz}$ is represented by 5 . Error bars represent standard errors. Dashed and dotted lines represent isolines for displacement and acceleration, respectively. The asymmetry observed between lower and upper arms of error bars and the curves in the lines connecting the dots are due to both axes being expressed in a log scale. 


\section{Magnitude of response}

The magnitude of response, measured by the number of actions potentials, increases with stimulus amplitude. The increase observed is very similar across different frequencies when stimulus amplitude is expressed as velocity (Figure 2.12). Indeed, the number of spikes detected during stimulus signals with a supra-threshold velocity of $2 \mathrm{~mm} / \mathrm{s}$ did not differ across frequencies (Figure 2.13) $\left(\mathrm{F}_{7.0,21.1}=1.29, \mathrm{p}=0.3012\right)$. 


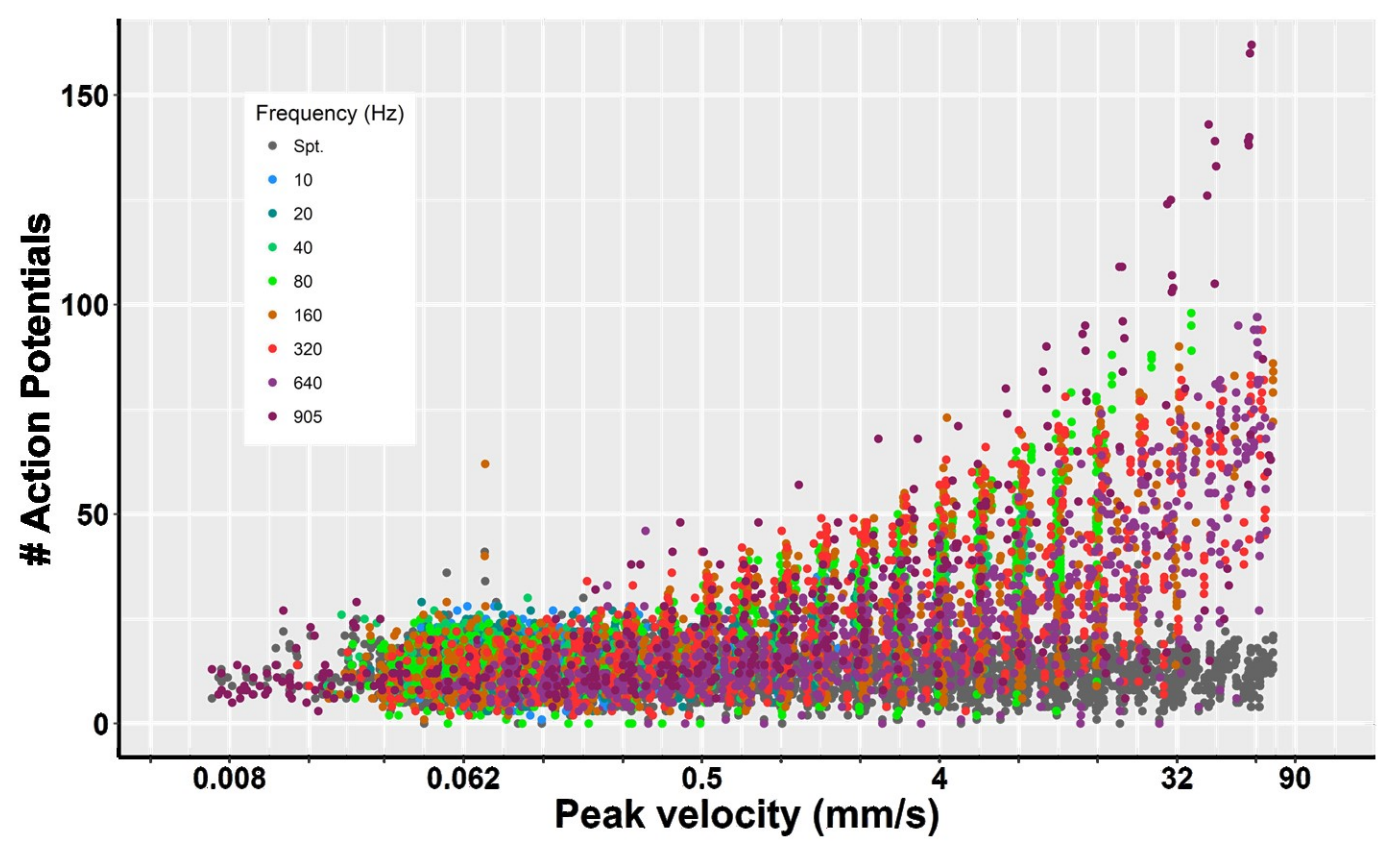

Figure 2.12 - Magnitude of response (\# action potentials) to increasing stimulus velocity. Whereas the background activity, represented by the number of action potentials before stimuli (grey dots), does not vary, the magnitude of response, represented by the number of action potentials during stimuli (coloured dots) increases with peak velocity of the stimulus. Frequencies from 10 to $640 \mathrm{~Hz}$ are represented by 8 animals whereas $905 \mathrm{~Hz}$ is represented by 5 . 


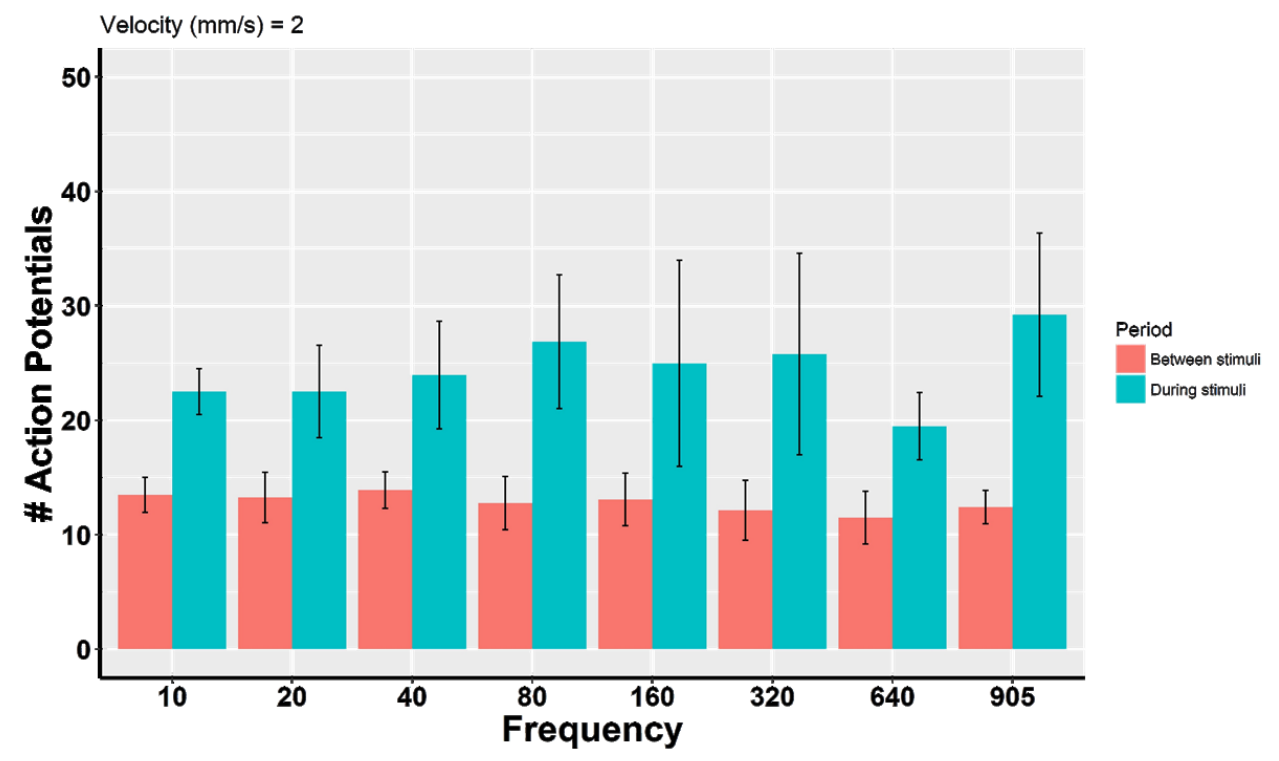

Figure 2.13 - Magnitude of response (\# action potentials) at supra-threshold stimuli of 2 $\mathrm{mm} / \mathrm{s}$ to different frequencies. Responses do not differ across frequencies $\left(\mathrm{F}_{7.0,21.1}=1.29\right.$, $\mathrm{p}=0.3012$ ). Blue and pink bars represent average number of action potentials detected during and before vibrational stimulus, respectively. Frequencies from 10 to $640 \mathrm{~Hz}$ are represented by 8 animals whereas $905 \mathrm{~Hz}$ is represented by 5. Error bars represent standard errors. 


\section{Directionality}

The hair presented a sensory response regardless of which orientation the step stimulus was applied. When a sine stimulus was applied, there was no significant difference between the two orthogonal directions, i.e. latero-medial and antero-posterior $\left(\mathrm{t}_{26.1}=-1.27\right.$; $\mathrm{p}=0.2160$ ). However, when a step stimulus was applied, the magnitude of response differed significantly across the four orientations $\left(\mathrm{F}_{3,90}=2.99, \mathrm{p}=0.0352\right)$, with the weakest response triggered when the hair was bent laterally and the strongest when it was bent medially. The number of action potentials happening during minus before each stimulus was $0.7 \pm 3.0$ and 2.6 \pm 3.3 , respectively. (Figure 2.14). 


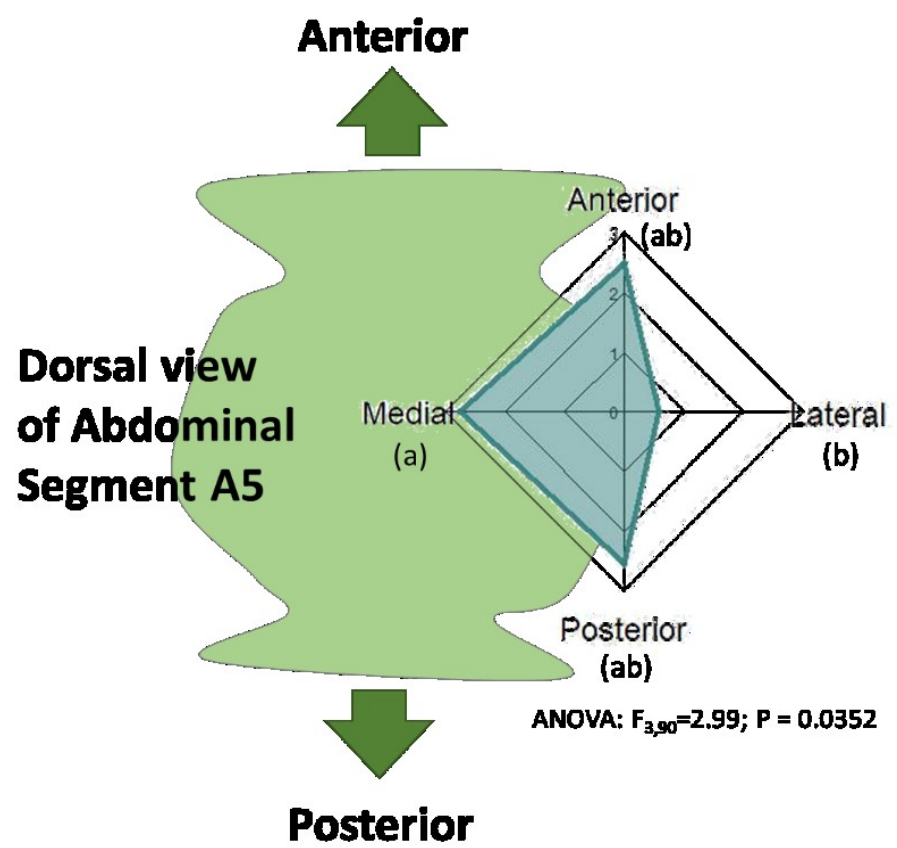

Figure 2.14 - Diagram representing the four orientations in which step stimuli were applied. The center of the radar plot represents the position of the hair relative to the abdomen. Blue lines in the radar plot indicate average number of spikes detected during minus before stimuli for a total of four individuals for medial and lateral orientations and only two for the posterior and anterior orientations. Directions accompanied by same letters between brackets did not differ significantly under a Tukey HSD test. 


\section{Rapid or slow adaptation (phasic or tonic)?}

The number of action potentials happening during different intervals of the step stimuli differed significantly $\left(\mathrm{H}_{3}=27.05, \mathrm{p}<0.0001\right)$. In fact, the rising slope of the stimulus, which is the first interval that happens right at the beginning, is significantly greater than the others, which do not differ significantly between themselves. Figure 2.15 shows the four distinct intervals and the neural response traces found during each interval. 


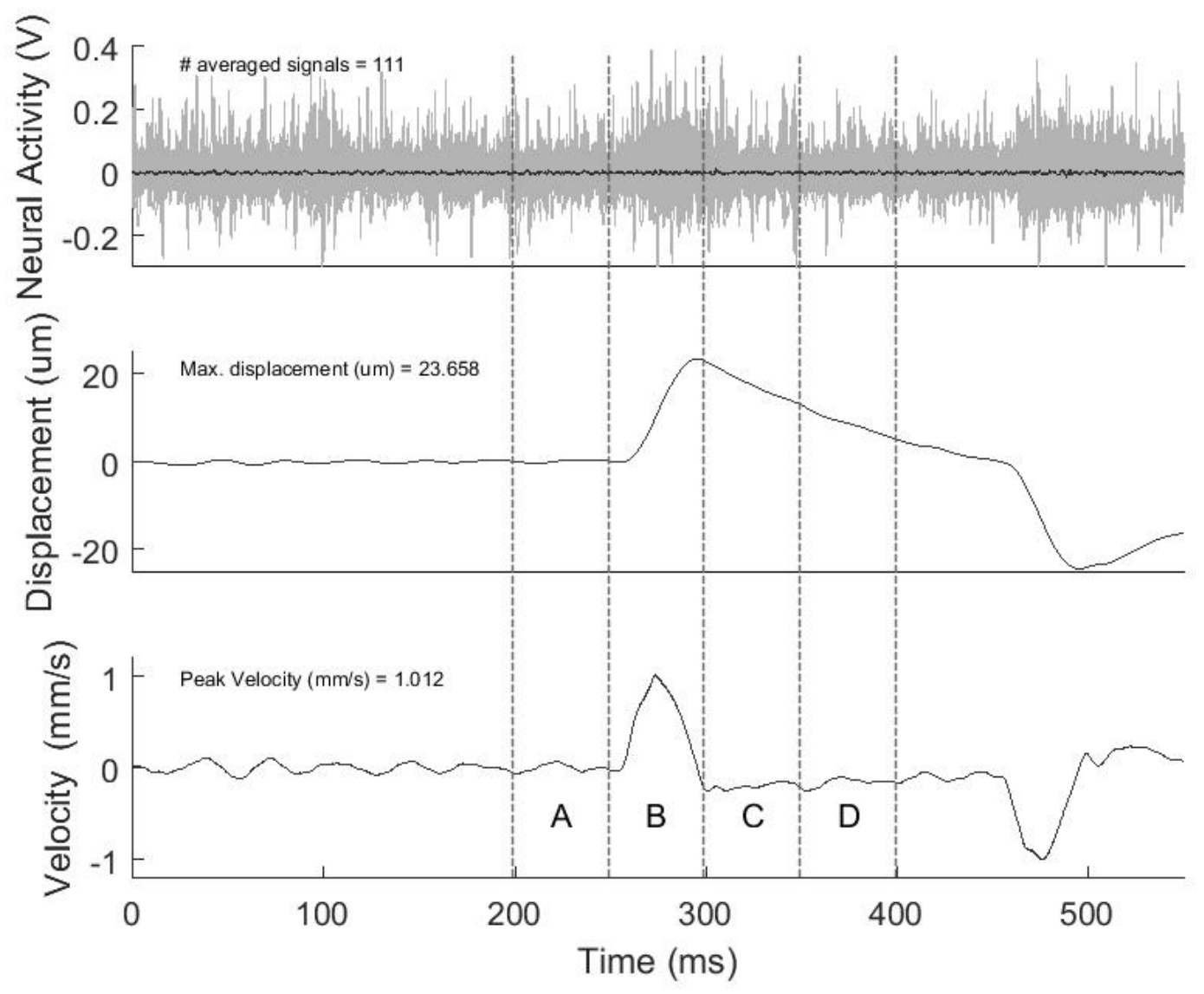

Figure 2.15 - The sensory hair shows a rapid adaptation to the stimulus. The two windows on the bottom show the step stimulus separated in four distinct intervals, A, B, $\mathrm{C}$ and $\mathrm{D}$, which are indicated by the letters between the dashed lines. The top window shows all responses overlaid (grey lines that look like a continuous grey area) and an average of all responses (dark line). The number of spikes happening during the $\mathrm{B}$ interval was 3.7, which was significantly higher than the numbers found in $\mathrm{A}, \mathrm{B}$ and $\mathrm{C}$, respectively of 2.3, 2.5 and 2.2, which do not differ between each other $\left(\mathrm{H}_{3}=27.05\right.$, $\mathrm{p}<0.0001)$. 


\section{Phase locking}

From the compiled responses, it is evident that some phase locking occurs. A remarkable example of phase locking is shown on Figure 2.16. However, the exact phase of the stimulus in which they occur is not consistent across different frequencies (Figure 2.17) (see Appendix A.5 - Phase locking for frequencies from 10 to $120 \mathrm{~Hz}$ in one $T$. ni) and neither across caterpillars (Figure 2.18) (see Appendix A.4 - Phase locking for frequencies 20,80 and $640 \mathrm{~Hz}$ in all $T$. $n i$ caterpillars). 

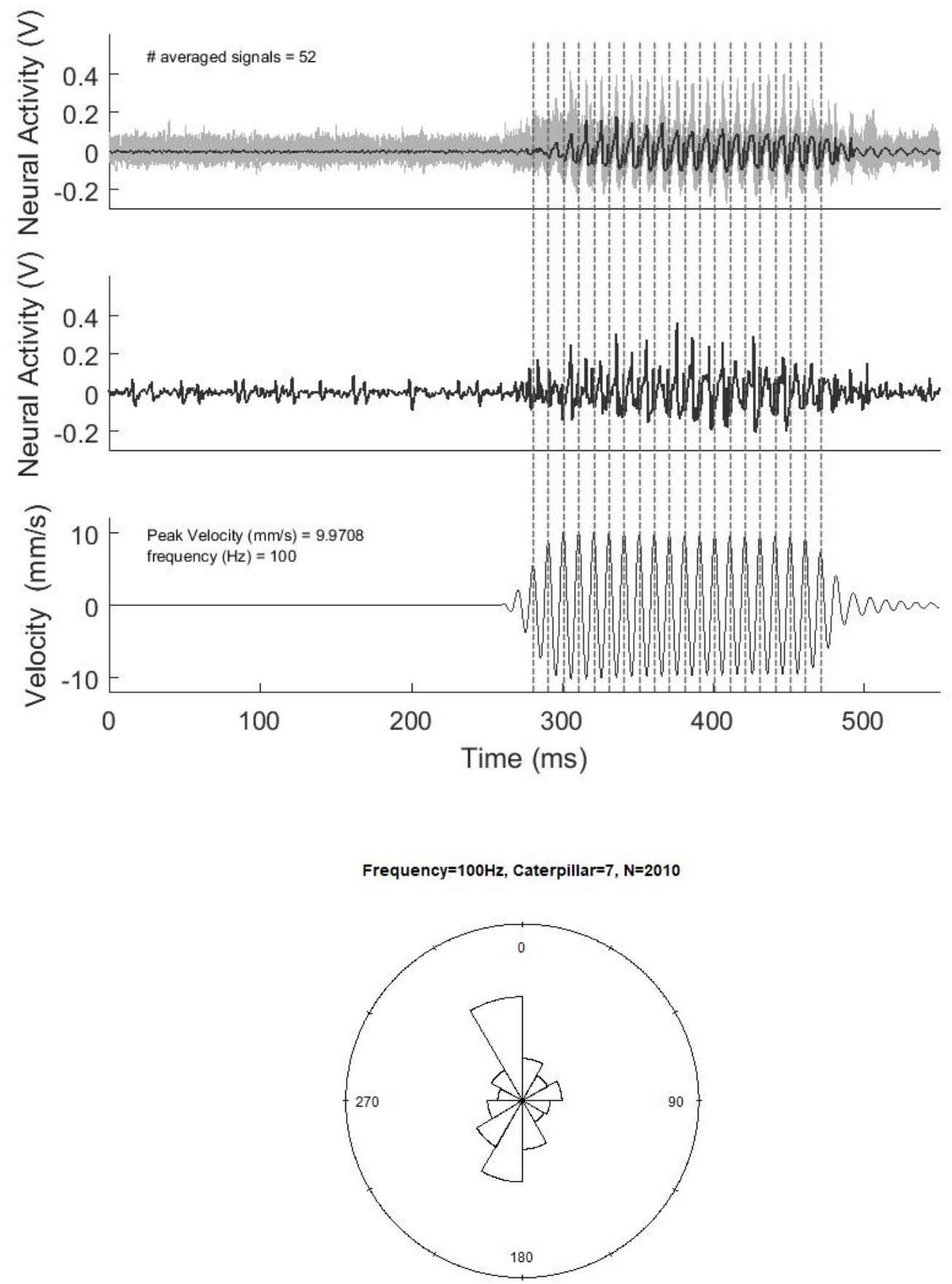

Bin size: 30 degrees

Figure 2.16 - Phase locking by sensory hair. The graph shows the vibrational signal that was applied repeatedly to the hair (bottom window), an individual trace of the response (middle window), all responses overlaid (grey lines that look like a continuous grey area 
on top window) and an average of all responses (dark line on top window). Dashed lines indicate the moments at which the stimulus reaches peak velocities, which correspond to a phase angle of $0 / 360^{\circ}$. The rose histogram below each graph summarizes the phases at which the spikes happened. The number of signals used in the analysis for each frequency is indicated on the top window of the graph, while the number of spikes counted is indicated on the title of the rose histogram. In this figure, it is clear that action potentials in response to $100 \mathrm{~Hz}$ sine signals happen predominantly at $0^{\circ}$ and $180^{\circ}$, which correspond to the peak velocities in both directions of the stimulus. The neural responses shown on the graph were recorded from a de-efferented ventral nerve, which means the recorded activity was sensory, not motor. A comparison between this rose histogram and those constructed from other frequencies is shown on Figure 2.17. 

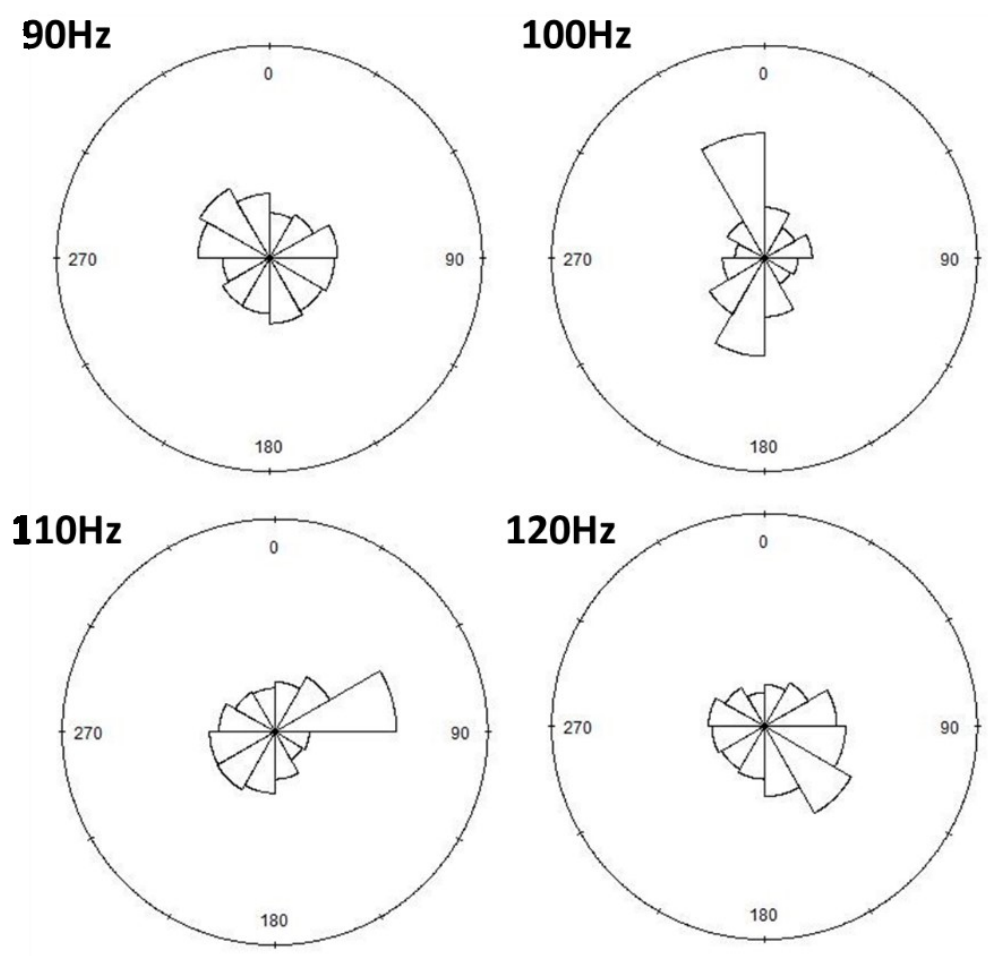

Figure 2.17 - Phase locking for 90, 100, 110 and $120 \mathrm{~Hz}$ in one T. ni caterpillar. In this figure, phase locking is visible in all frequencies, specially for 100 and $110 \mathrm{~Hz}$. However, the predominant phases differ across frequencies. All histograms were constructed using relative angles of action potentials that were recorded from the ventral nerve of the same caterpillar, which had been de-efferented. In addition to the rose histograms, sample traces of these and other frequencies from the same caterpillar can be found in the Appendix (Appendix A.5 - Phase locking for frequencies from 10 to $120 \mathrm{~Hz}$ in one $T$. ni caterpillar). 


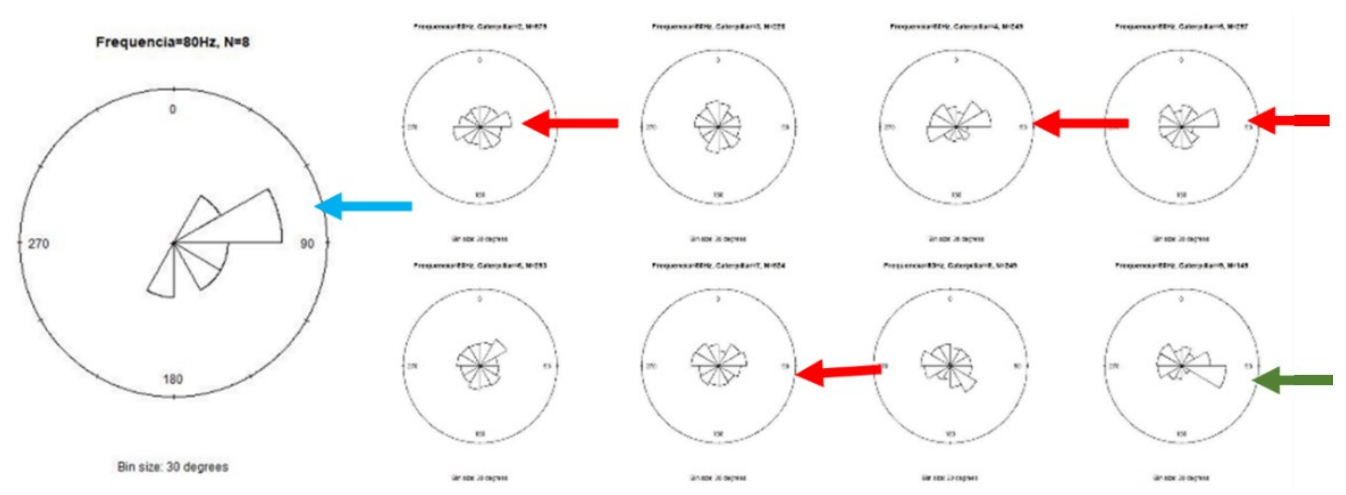

Figure 2.18 - Phase locking for $80 \mathrm{~Hz}$ in all $T$. $n i$ caterpillars. Assigned spike angles obtained from neural responses were plotted in rose histograms. Small rose histograms represent individual caterpillars and the large one summarizes the largest bins observed across caterpillars (blue arrow). Red arrows point individual caterpillars that contributed directly to the largest bin, whereas green arrows point caterpillars that did not contribute directly but still followed a similar pattern. It is visible from this figure that some phase locking occurs, although it is not consistent across all caterpillars. Rose histograms for all caterpillars submitted to 20 and $640 \mathrm{~Hz}$ can be found in the Appendix (Appendix A.4 Phase locking for frequencies 20,80 and $640 \mathrm{~Hz}$ in all $T$. $n$ i caterpillars). 


\section{Discussion}

In this study, I showed that the lateral hair on the proleg responds to vibrational stimuli from 10 to $905 \mathrm{~Hz}$. The thresholds for all frequencies were determined in the three quantities of magnitude: Displacement, Velocity and Acceleration. Interestingly, the velocity thresholds did not vary across frequencies, with averages falling between 0.5 and $0.9 \mathrm{~mm} / \mathrm{s}$ for all of them. These thresholds are higher than values previously described in the literature for velocity sensitive hairs, which varied from 0.03 to $0.3 \mathrm{~mm} / \mathrm{s}$, although in that case hairs were tested to detect near-field air-borne sounds in crickets (Shimozawa and Kanou, 1984). The values found here are also higher than thresholds for velocity receptors of the subgenual organs in honeybees, which varied from 0.03 to $0.1 \mathrm{~mm} / \mathrm{s}$, depending on the direction in which the vibrational stimulus was applied (Cocroft, 2016). Therefore, the lateral hair tested here was shown to be less sensitive to vibration than other velocity receptors in insects, although methods differed greatly between studies as well as the organs and contexts in which they are used. Nevertheless, the sensitivity of the hair as found here would theoretically be enough to detect biotic and abiotic vibrations as measured on the leaf(Table 1.1).

The magnitude of neural response, i.e. number of action potentials, increased with the amplitude of the stimulus. Such an increase has been previously found in other substrate-borne vibration receptors such as the tibial chordotonal organ and the subgenual organ in Mantophasmatodea (Eberhard et al., 2010). However, in the result here presented the increase was highly correlated with the peak velocity of the stimulus and was extremely consistent across all frequencies. This is interesting, considering that the sine stimuli applied had the same duration for all frequencies, of $200 \mathrm{~ms}$ plus $25 \mathrm{~ms}$ of 
rising and decaying phases. This resulted in different number of wave cycles applied to the hair, depending on the frequency.

For example, whereas the $10 \mathrm{~Hz}$ signals only included two wave cycles, the $80 \mathrm{~Hz}$ included 16 and the $640 \mathrm{~Hz}$ included 128. At first glance, more wave cycles with the same peak velocity would presumably trigger more action potentials. But at a deeper analysis, the period of each wave from higher frequencies is also shorter, following an inverse proportionality relationship with the number of waves. Therefore, the result that I found might indicate that the number of action potentials triggered by one single wave cycle is determined by a function of its peak velocity and period. In other words, the number of action potentials depend on the peak velocity of a stimulus and for how long this velocity is applied. The step stimulus experiment could have aided in clarifying this conclusion, but unfortunately its set-up did not allow me to produce peak velocities that were much higher than the threshold. However, the step stimulus experiment did show that the hair has a rapid adapting response that happens while a velocity is applied, supporting this conclusion.

Being a velocity receptor does not necessarily mean that the sensory hair is in fact used by the caterpillar to perceive vibrations. For example, tactile hairs in spiders can be velocity receptors as well (Barth, 2004). In fact, my directionality experiment revealed that the hair is most sensitive to step stimuli applied medially from the side, i.e. towards the proleg. This could be evidence that the hair in question serves as a tactile receptor, possibly to detect objects that approach the proleg from the side or to aid the caterpillar is crawling (van Griethuijsen and Trimmer, 2010). 
In contrast, directionality in sensitive hairs to air-borne sound was shown to be a way to localize the direction of the sound source (Shimozawa and Kanou, 1984). One single hair in this case is not enough: it would be necessary to have several hairs with similar thresholds but different directionality sensitivities to infer where the sound stimulus is coming from (Shimozawa and Kanou, 1984). In crickets, while the external morphology of hairs is associated with their neural response, hairs with similar lengths have similar thresholds (Shimozawa and Kanou, 1984). In my caterpillar, each proleg has three hairs - each touching the leaf at a different direction. Assuming from their similar morphologies and lengths that they could have similar velocity thresholds as well, maybe these hairs serve to detect and localize the source of a substrate-borne vibration. The rapid adapting response in addition to the phase locking found here, could aid in this process. However, this cannot be concluded from the experiments yet.

In conclusion, I have shown that the hair on the proleg is sensory. Not only that, but I also showed that it can detect substrate-borne vibration, specifically as a velocity receptor, and that its neural response contains information about the stimulus duration and amplitude. The hair has directional sensitivity and shows neural responses at phases of the stimulus that have higher velocities, although a consistent phase locking did not occur. Finally, the thresholds of the hair are low enough to detect substrate-borne vibrations that are naturally found in leaves (Guedes et al., 2012). For further study, it remains to be confirmed if the hair is indeed used by the caterpillar for perceiving substrate-borne vibrations. This could be done by behavioural experiments, ablations, and playback experiments. 


\section{Chapter 3 Vibration detection in Drepana arcuata: insights from one caterpillar}

\section{Introduction}

Drepana arcuata (Drepanidae) is a Lepidoptera species whose larvae, the Masked Birch Caterpillars, feed on Birch (Betula) and Alder (Alnus) trees in North America. It is well evidenced that they can detect and respond selectively to complex vibratory signals (Yack et al., 2001; Guedes et al., 2012). The vibrational repertoire used by D. arcuata caterpillars includes at least four different signals, shown to communicate to conspecifics. They are produced by moving or striking body parts against the leaves (Yack et al., 2001; Scott et al., 2010; Guedes et al., 2012; Yadav et al., 2017). These caterpillars are also believed to detect leaf borne vibrations transmitted by crawling conspecifics and hemipteran predators, as well as wind and rain (Guedes et al., 2012). All signals produced during territorial disputes have low dominant frequencies, generally between 10 and 60 $\mathrm{Hz}$, but the energy in the top $10 \mathrm{~dB}$ can extend up to $200 \mathrm{~Hz}$ (Guedes et al., 2012). Depending on the type of signal, peak velocities of vibrations measured at about $20 \mathrm{~mm}$ from the source can vary between 2.0 and $50.0 \mathrm{~mm} / \mathrm{s}$ (Guedes et al., 2012). Therefore, vibration receptors of $D$. arcuata caterpillars are likely to be sensitive to frequencies and amplitudes at this range.

Similarly to Trichoplusia $n i$, the prolegs of $D$. arcuata caterpillars contain three thick hairs that are in constant contact with the leaf surface (Figure 3.1 and Figure 3.2). In an unpublished observation by I. Hassenfuss (personal communication with JEY), the hairs were named SV1, SV2 and V1 using nomenclature system designed by Hinton 
(1946) (Figure 3.2). Also in this observation, at least two chordotonal organs were noted and illustrated in the prolegs (Figure 3.2). However, the presence of these chordotonal organs needs to be confirmed with further morphological investigations. The anatomical and neurophysiological experiments that will be described below focus on the sensory hairs and possibly chordotonal organs of the prolegs. 

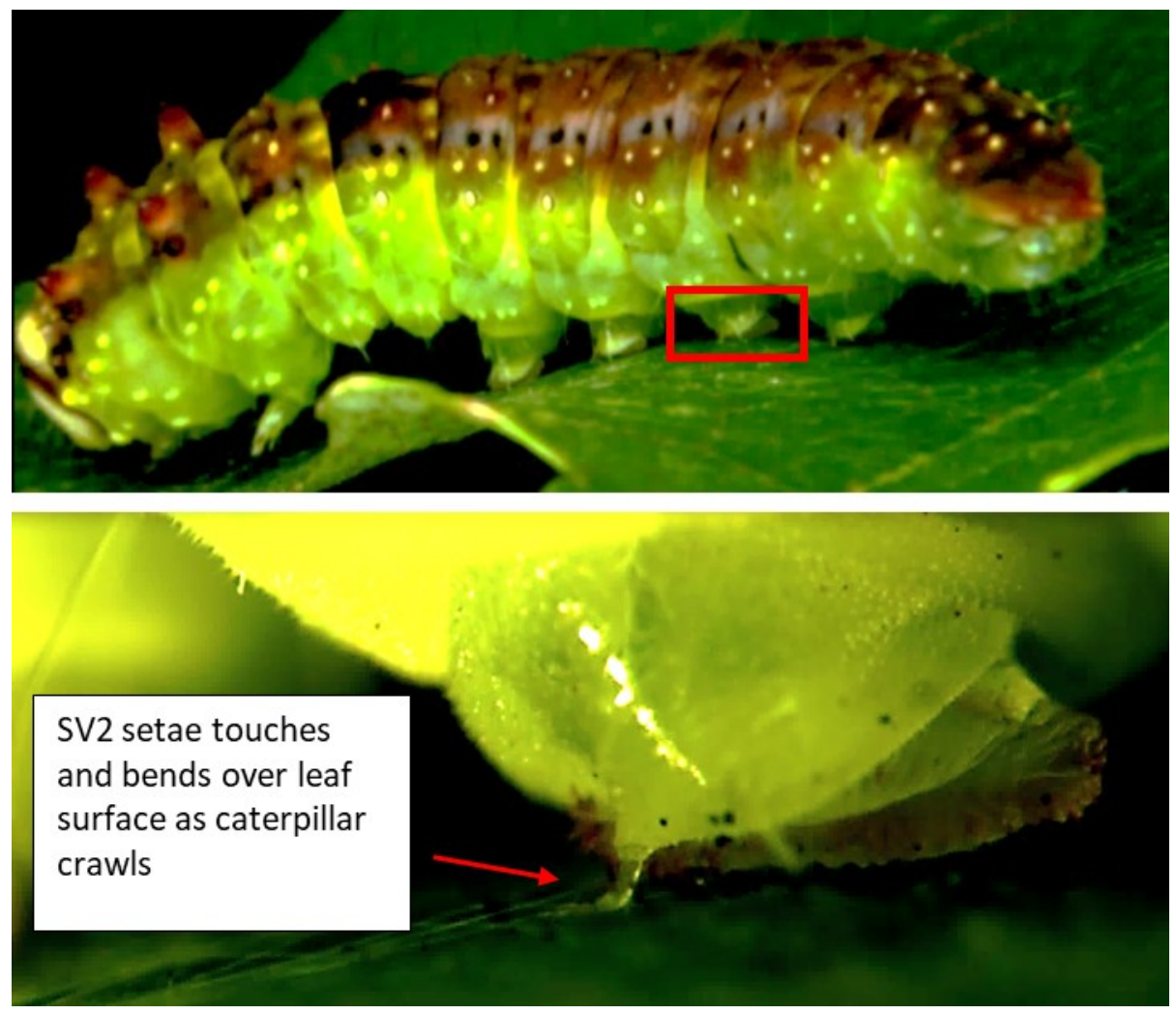

Figure 3.1 - In D. arcuata, proleg hairs are often in direct contact with the leaf surface. 

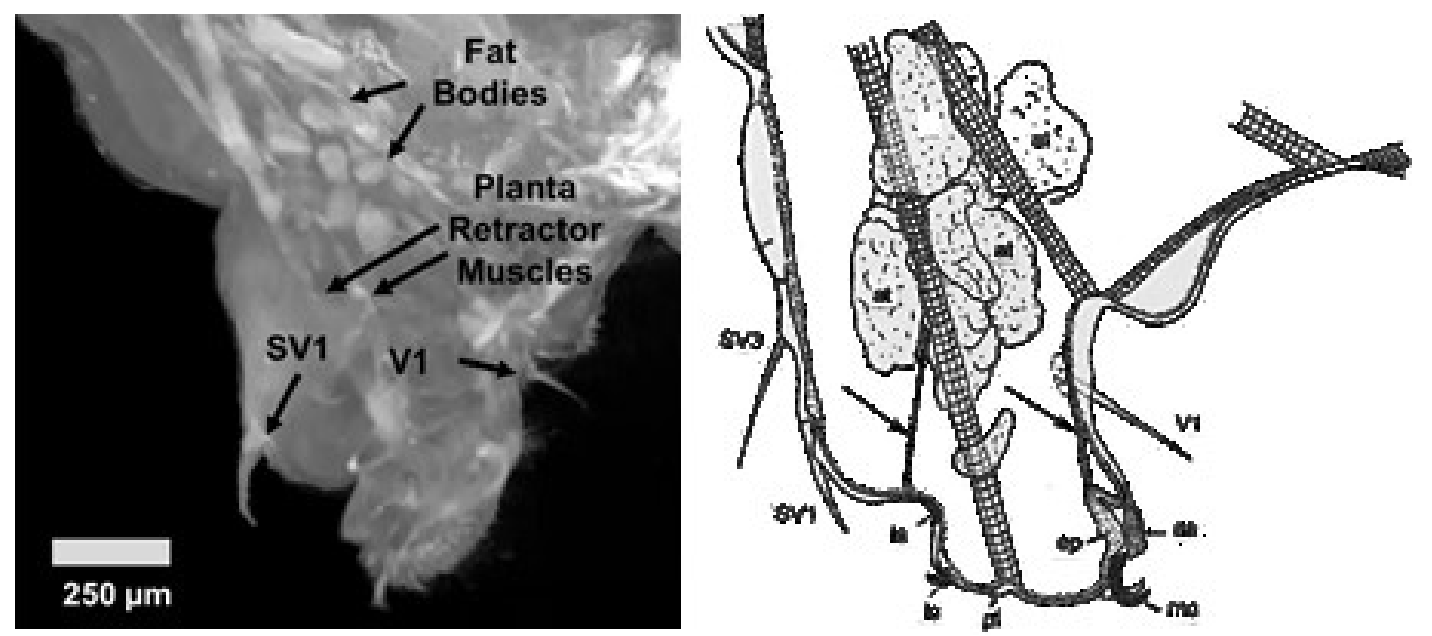

Figure 3.2 - Internal anatomy of a $D$. arcuata proleg. Left - Micro-CT of a D. arcuata proleg showing hairs SV1 and V1 and muscles and fat bodies. Right - Illustration by I. Hassenfuss (personal communication with JEY) showing the same hairs and arrows pointing potential chordotonal organs. Micro Computed Tomography was performed by myself and technical details are briefly described in the Appendix (Appendix C.1 Technical details on Micro-CT scanning). 


\section{Materials and Methods}

\section{Animals}

Gravid females of D. arcuata were collected in the wild as moths and larvae were reared from eggs on Paper Birch (Betula papyfera). Two caterpillars in the $5^{\text {th }}$ instar were used in the following experiments.

\section{Specimen preparation and neural recording}

The dissection procedure for exposing the ganglion and hooking the ventral nerve with stainless steel electrodes was the same as that described in Chapter 2 of this thesis.

\section{Stimulus application and calibration}

Most of the methods for stimulus application, calibration and recording were the same as that of Chapter 2. However, the experiment described in this chapter was executed before that of Chapter 2 and a couple aspects of these methods are not as polished. First, here the mirrored tape where the laser was pointed to was not positioned at the forceps but rather on the surface of the vibrating plate of the Mini-shaker. Prospective experiments revealed that this variation in the position of the mirrored tape did not affect significantly the frequency and amplitude of the stimulus detected by the laser. Second, here only two $D$. arcuata caterpillars were used and, whereas in one caterpillar the forceps used to grip the SV1 hair was the same as that in Chapter 2, in the other D. arcuata caterpillar an orange wood stick was used instead. The wooden stick was waxed to a screw attached to the Mini-shaker. The tip of the orange stick was covered with melted wax and gently approached to the hair using the micromanipulators that supported the Mini-shaker. 
However, the wax did not only cover SV1 seta, but also SV2 and part of the cuticle in between them.

\section{Stimulus}

Only signals of $10 \mathrm{~Hz}$ could be applied by the forceps to the hair of one caterpillar before the neural activity of the nerve faded off. For the other caterpillar, signals with precalculated amplitudes and frequencies ranging from 10 to $640 \mathrm{~Hz}$ could be applied by the waxed wooden stick to its hairs and cuticle. All signals were applied in the medial-lateral orientation and no step stimulus was used in these experiments.

\section{Statistical analysis}

Thresholds were calculated in the same way as that described in Chapter 2, using local regressions with $99 \%$ confidence intervals. Graphs for local regressions are shown in the Appendix (Appendix B.1 - Local regressions loess() for all frequencies in two $D$. arcuata caterpillars). Since a broad range of frequencies could only be applied to one caterpillar, it was not possible to test statistical significance of differences in thresholds across frequencies, but the complete sensitivity curve could be plotted and examined for this individual. 


\section{Results and Discussion}

Sensory responses to vibrational stimuli of different intensities, ranging from subto supra-threshold, could be recorded in the waxed cuticle of the proleg (Figure 3.3). Responses to all applied frequencies are found in the Appendix (Appendix B.2 - Sample traces of neural activity from waxed cuticle in $D$. arcuata). Sensory responses to sensory hair submitted to $10 \mathrm{~Hz}$ vibrations are also found in the appendix (Appendix B.3

- Sample traces of neural activity from a single proleg hair in D. arcuata). The thresholds for vibration detection found in D. arcuata seem to vary across frequencies. Regardless of which proxy of amplitude is considered, the two hairs plus cuticle stimulated showed highest sensitivity to vibration frequencies from 40 and $320 \mathrm{~Hz}$ (Figure 3.4). The lowest thresholds in displacement, velocity and acceleration found were $22 \mathrm{~nm}, 0.012 \mathrm{~mm} / \mathrm{s}$ and $0.07 \mathrm{~m} / \mathrm{s}^{2}$, all obtained in response to $160 \mathrm{~Hz}$ vibrations. The velocity thresholds obtained here are one order of magnitude lower than those obtained for the proleg hair of $T$. ni. However, a direct comparison is not possible since the proleg cuticle of $T$. $n i$ had not been stimulated in Chapter 2, differently from what happened in this Chapter, where the cuticle was accidentally covered by the wax and stimulated along with the hairs. 

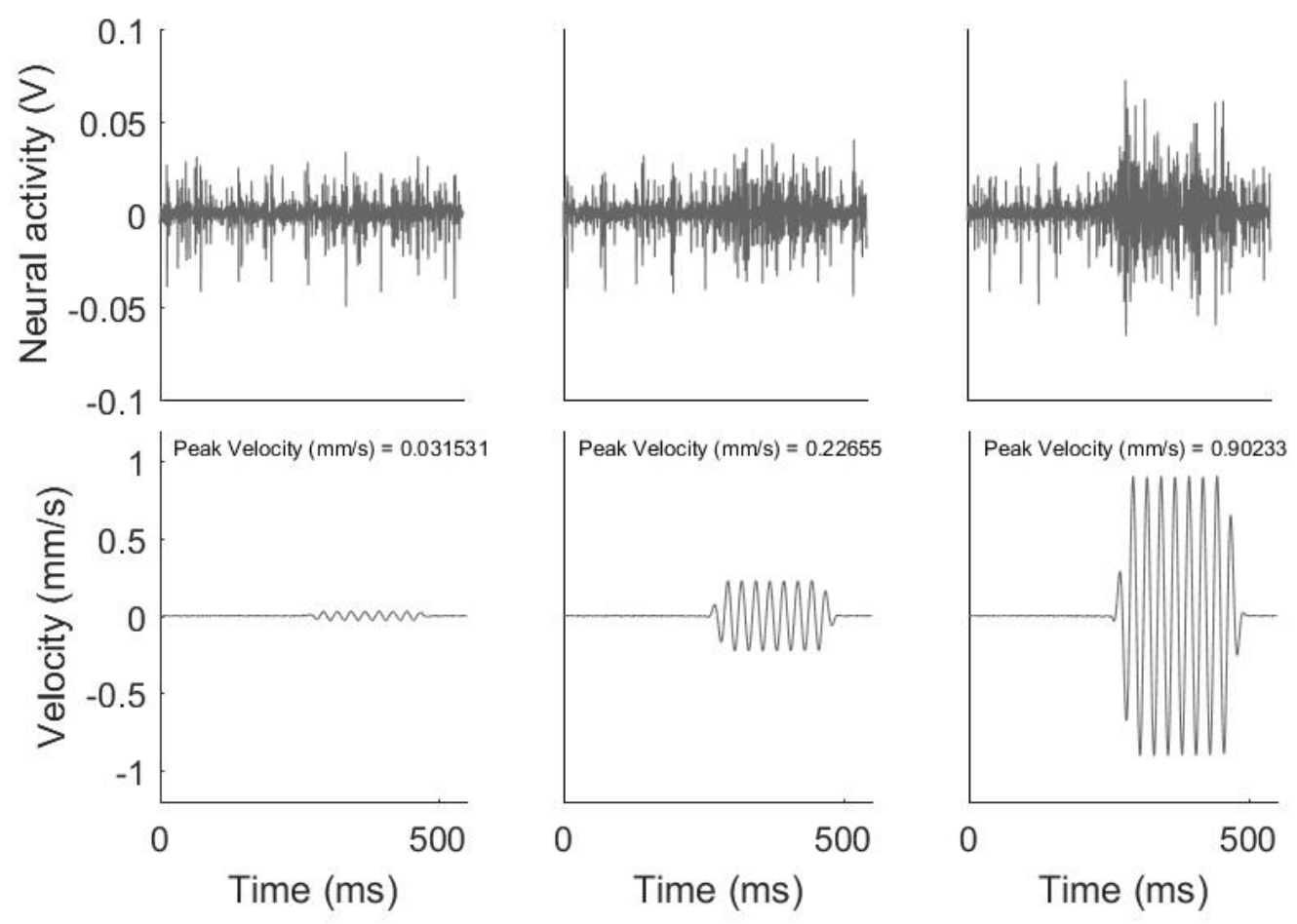

Figure 3.3 - Sensory responses to vibration by the waxed cuticle and hair of D. arcuata. 


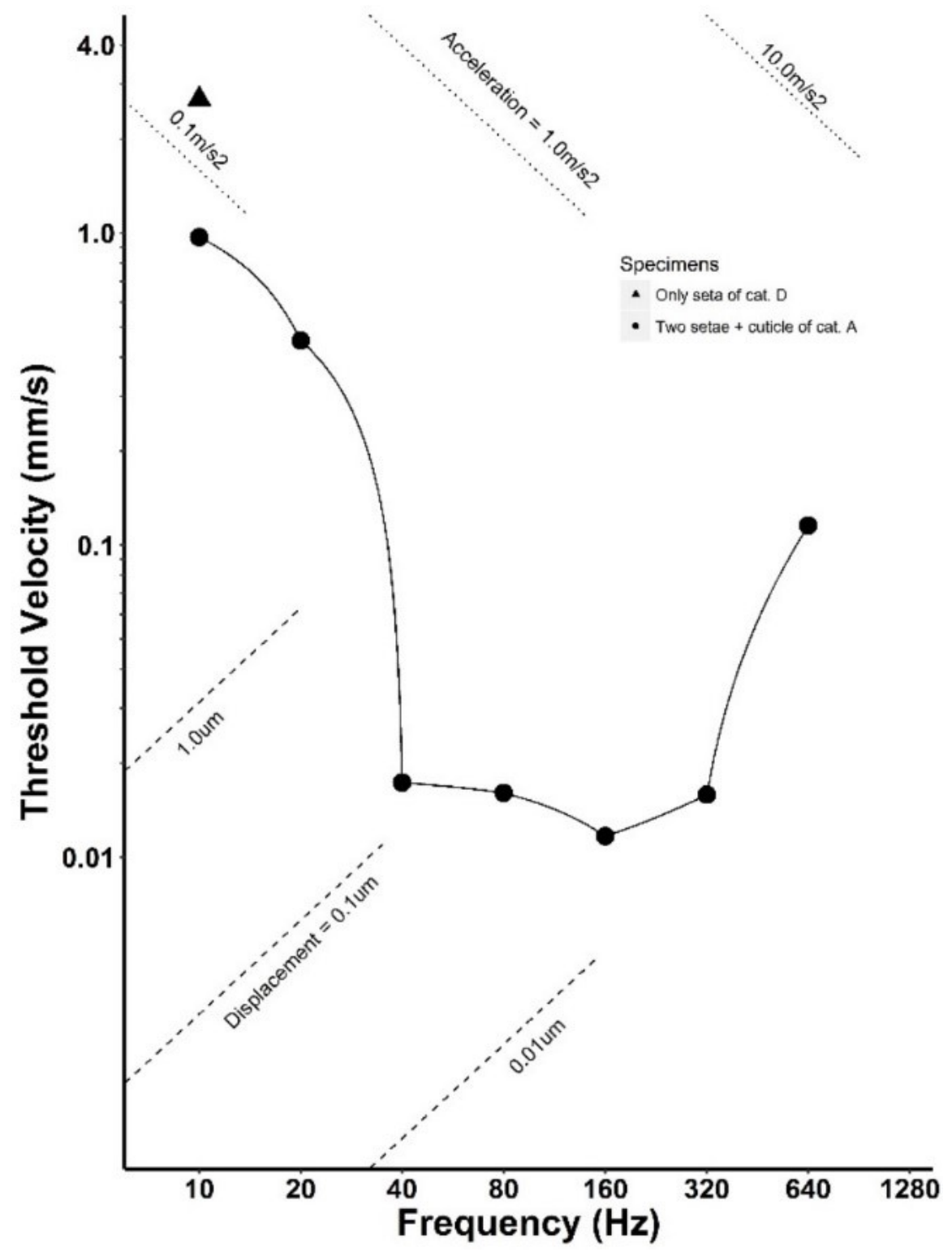

Figure 3.4 - Thresholds of neural response obtained from the two D. arcuata caterpillars. The single triangle indicates the threshold to $10 \mathrm{~Hz}$ vibrations of the hair from one caterpillar stimulated using the forceps. The round dots connected by lines indicate thresholds across frequencies of the other caterpillar, which had its two setae and part of the cuticle covered by wax during vibration stimulation. 


\section{Chapter 4 Tutorial for dissecting, exposing and recording from ventral nerve in the abdominal ganglion of Drepana arcuata}

\section{Statement of purpose}

Dissecting caterpillars for neurophysiological purposes represented a challenge throughout my thesis. A great part of the time was invested in learning how to expose the nervous system of these soft bodied insects and failing to do so. Incisions on the cuticle and tissues manipulation must be executed carefully to avoid excessive leakage of hemolymph, which may cause reduction in the internal hydrostatic pressure and deflation of body parts, including the prolegs. Moreover, these insects can make frequent contraction movements during dissection, even after their body is ligated and cut in half. These movements can make it more difficult to conduct dissections and make neurophysiological recordings and eventually they cause hemolymph leakage and nervous system displacement along the body, reducing the life spam and quality of neural recordings.

The purpose of this Chapter is to provide a short protocol with illustrations about how to expose and hook on the ventral nerve of the abdominal ganglion of a Drepana arcuata $5^{\text {th }}$ instar caterpillar. I also provide a short dissection video tutorial in which the whole protocol was executed successfully, terminating with the hooking and neural recording of the ventral nerve. 


\section{Materials and Methods}

A D. arcuata caterpillar was dissected under a stereomicroscope. The whole procedure was filmed using two cameras simultaneously. The first (DMC4500, Leica Microsystems $\mathrm{GmbH}$ ) was attached to the microscope (M205C, Leica Microsystems

GmbH, Wetzlar, Germany) and filmed the dissection from above with a close-up perspective. The other (HDR-HC7, Sony, Tokyo, Japan) was located beside and filmed the manipulation of tools and the caterpillar from a farther perspective. Videos filmed by both cameras were overlaid using iMovie 10 (Apple, Cupertino, USA) and edited to reduce its duration from 40 to 5 minutes without missing the key points of the dissection process. 


\section{Results}

An illustrative figure was made to show the relative position of the abdominal ganglion to the proleg (Figure 4.1) and the dissection protocol is written below (Protocol for physiology on prolegs). The video tutorial was successful as it showed the key steps for dissecting and exposing the abdominal ganglion and hooking an electrode on the ventral nerve. Screenshots of the tutorial video that correspond to specific steps in the written protocol are shown (Figure 4.2). 


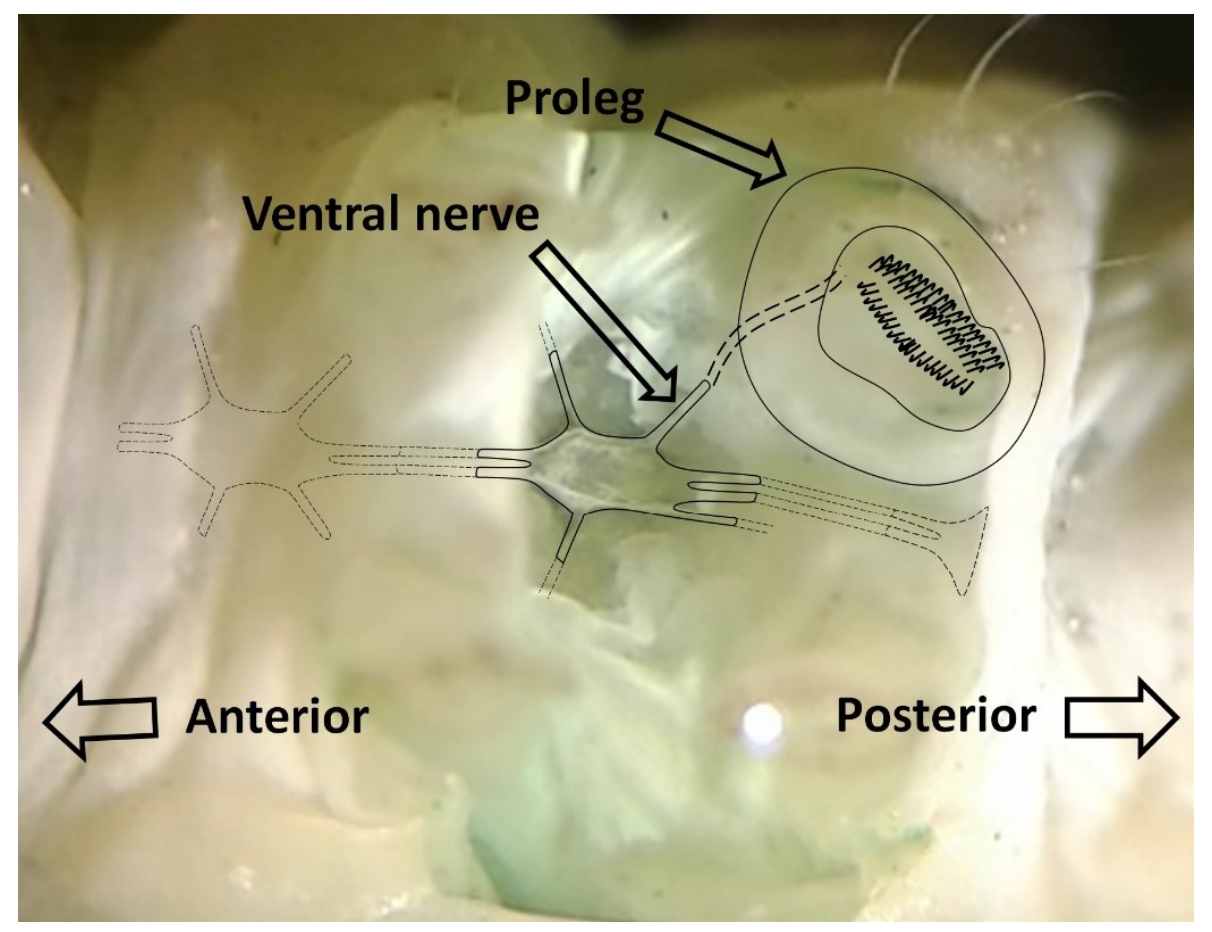

Figure 4.1 - The ventral nerve of an abdominal ganglion. Each abdominal ganglion has two ventral nerves, each of which innervates one proleg. 


\section{Protocol for physiology on prolegs}

1- To help anesthetizing, put living caterpillar in the $4^{\circ} \mathrm{C}$ fridge for a couple minutes. If wished, the fridge may also be used to slow down the caterpillar`s development during a few hours or days. If that is the case, some care is recommended:

a. Caterpillar must be inside a glass or plastic vial.

b. Vial must have a cover.

c. Humidity inside vial must be high. If necessary, place a soaked paper towel inside it.

d. Vial may contain food - leaf or diet. Reason: Some caterpillars keep chewing even in the fridge.

e. Under these conditions, caterpillars can stay in the fridge from a few minutes to a few days, although it seems that the longer they stay in the fridge the faster their neural activity fades off during neurophysiological experiments;

2- Take caterpillar out of the fridge;

3- Anesthetize it with $\mathrm{CO}_{2}$ by putting the caterpillar on the $\mathrm{CO}_{2}$ emitter plate and covering it with a petri dish.

4- $\quad$ Tie the mid body up, between segments A2 and A3;

5- Cut thorax off

a. You may leave a piece of cuticle so that you can have some extra tissue to pin it by later. 
6- Clean fluids up;

7- $\quad$ Pin abdomen down;

8- Shorten non-targeted sensory hairs;

a. This step helps keeping caterpillar calmer.

9- $\quad$ Make a deep incision anteriorly on the abdomen, close to where it is tied up. Obs.: This step is optional, but not executing it might cause some troubles later, as it is demonstrated in a supplementary video in the Appendix (Appendix D.3 - What might happen when the caterpillar wiggles).

a. The incision must be deep enough to make a hole in the gut.

b. The reason is to release pressure from the gut so that it does not puff outwards later;

c. See Step 11.d "Clean way up to ganglion - Gut" for more details.

10- Make superficial incision ventrally to the ganglion;

a. Sometimes the ganglia can be seen from outside the cuticle, but not always.

b. The exact location of a ganglion may vary because the nervous system moves along the anteroposterior axis as caterpillar wiggles.

c. The most common location of a ganglion is immediately anterior to the point between prolegs that it innervates.

11- Clean way up to ganglion;

a. Fat bodies: 
i. Look like: Small pieces of light yellow soft tissue that are found floating everywhere. They have irregular shapes that look porous and sparkle under the light.

ii. To clean: grab them by small amounts and clean forceps on a disposable wiper that had been soaked with alcohol. Dry forceps up on a dry part of disposable wiper.

b. Salivary glands:

i. Look like: Very thick semi-transparent cylindrical tubes. The is one is located on each side of body.

ii. To clean: Do not pull it. These glands run along the whole length of the body. Trying to pull them barely works and may damage several tissues around. If one of them is on the way - touching the ganglion or the nerve hold it firmly with the forceps and cut it with the scissors at the furthest points inside that you can - anteriorly and posteriorly.

c. Malpighian tubules:

i. Look like: Dozens of tangled tiny tubes, light yellow coloured.

ii. To clean: When damaged, Malpighian tubules release a lightcoloured opaque fluid that spreads easily and makes everything around hard to be seen. If they are on the way, first drip saline on them and use the forceps to grab the largest amount that you can. Clean the fluid released with more saline and repeat the process until the way is clean of Malpighian tubules. 
d. Gut:

i. Look like: if caterpillar fed on leaves, it is dark green. If fed on diet, it is light brown. For some reason, it is hard to define the exact boundary of the gut. Usually the gut is only noticeable when the caterpillar has moved a lot and the pressure inside the gut makes it puff outwards through the incision, touching and embracing the ganglion and the nerves.

ii. To clean: The ideal is to have taken step 9 at the beginning of dissection. If step 9 had not been taken or if it had but the gut still puffed towards the ganglion, don't penetrate, pull, cut or touch the gut with neither the forceps or the scissors. Its cuticle is fragile, and any damage may cause its content to spread through the whole body, including lots of food and enzymes. Most times that this happened I ended up losing the specimen. Instead, use scissors to deepen the incision made during Step 9 and use the forceps to gently pull the gut out from this incision. This procedure is demonstrated in a supplementary video in the Appendix

\section{(Appendix D.4 - How to do deflate the gut).}

12- Identify ventral nerve(s);

a. Ventral nerves innervate the ventral part of their respective segment, including the prolegs.

b. There is one ventral nerve on each side of ganglion. Each nerve innervates the proleg ipsilateral to it - i.e. left nerve innervates left proleg. 
c. The ventral nerves are located posteriorly on the abdominal ganglion.

13- Separate nerve from trachea;

a. There is a trachea running all the way along the ventral nerve, in very close contact to it. Whenever the nerve bifurcates, the trachea also bifurcates.

b. To record action potentials from the nerve, the trachea must be separated so that the electrode hooks on the nerve alone. Hooking the steel electrode on the nerve together with the trachea never gave me successful recordings.

c. Separating trachea from nerve has been the hardest step for me.

d. The procedure requires a lot of precision and care. When holding the forceps by hand, the nerve can be easily damaged and cut off by accidental movements.

e. For this reason, I suggest using the electrodes on the micromanipulators.

f. The Recording and Reference electrodes can be used together to separate nerve from trachea.

g. The trachea may be cut to avoid that both tissues merge again.

14- Hook nerve with Recording electrode;

15- Insert Reference electrode in the saline;

16- Raise the Recording electrode above saline to start recording. 

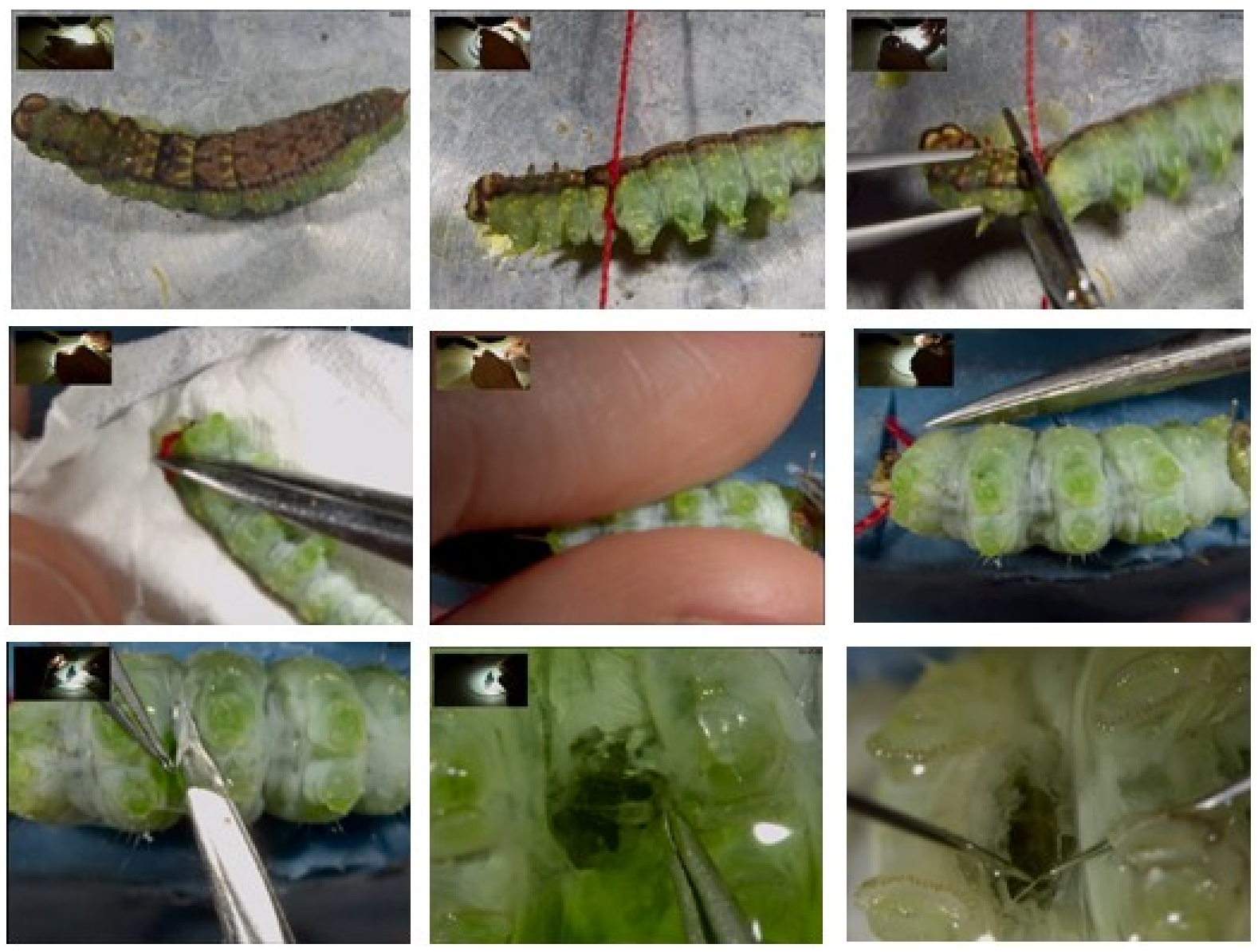

Figure 4.2 - Screenshots from the tutorial video. From left to right, top to bottom, images correspond to steps $3,4,5,6,7,8,10,11$ and 12 of the written protocol, respectively. The tutorial video is found in the Appendix (Appendix D.1 - Short tutorial for exposing a caterpillar’s nervous ganglion). 


\section{Chapter 5 Final remarks}

\section{Overall Conclusions}

In this work, I showed that the sensory response of proleg hairs in $T$. $n i$ can be characterized as a rapid adapting response, that it has constant thresholds across frequencies in the velocity proxy, that the number of action potentials increases with the stimulus peak-velocity, that action potentials have some degree of phase locking, and that sensitivity is directional. The proleg hair in $D$. arcuata also responds to vibration, although its response could not be characterized in so many details. The sensory hairs here evaluated can respond to vibration, which leaves them as potential candidates for substrate-borne vibration receptors in these caterpillars. 


\section{References}

Ai, H., Rybak, J., Menzel, R., \& Itoh, T. (2009). Response characteristics of vibration-sensitive interneurons related to Johnston's organ in the honeybee, Apis mellifera. Journal of Comparative Neurology, 515(2), 145-160.

Albert, P. J. (1980). Morphology and innervation of mouthpart sensilla in larvae of the spruce budworm, Choristoneura fumiferana (Clem.) (Lepidoptera: Tortricidae). Canadian Journal of Zoology, 58(5), 842-851.

Auld, B.A. (1973). Acoustic fields and waves in solids. Wiley Interscience Pub., 278.

Bacher, S., Casas, J., \& Dorn, S. (1996). Parasitoid vibrations as potential releasing stimulus of evasive behaviour in a leafminer. Physiological Entomology, 21(1), $33-43$.

Barth, F.G. (2004). Spider mechanoreceptors. Current Opinion in Neurobiology, 14(4), $415-422$.

Bavi, N., Richardson, J., \& Poole, K. (2018). Cellular mechanotransduction via on channels at the cell-substrate interface. Biophysical Journal, 114(3), 19a.

Bokolia, N.P., \& Mishra, M. (2015). Hearing molecules, mechanism and transportation: modeled in Drosophila melanogaster. Developmental Neurobiology, 75(2), 109130.

Bowen, J.L., Mahony, S.J., Mason, A.C. \& Yack, J.E. (2008). Vibration-mediated territoriality in the warty birch caterpillar Drepana bilineata. Physiological Entomology. 33, 238-250.

Burke, W. (1954). An organ for proprioception and vibration sense in Carcinus maenas. Journal of Experimental Biology, 31(1), 127-138.

Castellanos, I. \& Barbosa, P. (2006). Evaluation of predation risk by a caterpillar using substrate-borne vibrations. Animal Behaviour. 72, 461-469.

Cocroft, R.B. \& Rodríguez, R.L. (2005). The behavioral ecology of insect vibrational communication. BioScience. 55, 323-334.

Cocroft, R.B. (1999). Offspring-parent communication in a subsocial treehopper (Hemiptera: Membracidae: Umbonia crassicornis). Behaviour, 136(1), 1-21. 
Cocroft, R.B. (2016). Studying vibrational communication. M. Gogala, P. S. Hill, \& A. Wessel (Eds.). Springer.

Cocroft, R.B., Hamel, J., Su, Q., \& Gibson, J. (2014). Vibrational playback experiments: challenges and solutions. In Studying vibrational communication (pp. 249-274). Springer, Berlin, Heidelberg.

Čokl, A. (1983). Functional properties of vibroreceptors in the legs of Nezara viridula (L.) (Heteroptera, Pentatomidae). Journal of Comparative Physiology A: Neuroethology, Sensory, Neural, and Behavioral Physiology, 150(2), 261-269.

Čokl, A., Virant-Doberlet, M., \& McDowell, A. (1999). Vibrational directionality in the southern green stink bug, Nezara viridula (L.), is mediated by female song. Animal Behaviour, 58(6), 1277-1283.

Cummings, D.L.D., Gamboa, G.J. \& Harding, B.J. (1999). Lateral vibrations by social wasps signal larvae to withhold salivary secretions (Polistes fuscatus, Hymenoptera: Vespidae). Journal of Insect Behavior. 12, 465-473.

de Mendiburu, F (2016). agricolae: Statistical procedures for agricultural research. R package version 1.2-4. https://CRAN.R-project.org/package=agricolae.

Devetak, D. (1998). Detection of substrate vibration in Neuropteroidea: a review. Acta Zoologica Fennica, 209, 87-94.

Duggan, A., García-Añoveros, J., \& Corey, D. P. (2000). Insect mechanoreception: What a long, strange TRP it's been. Current Biology, 10(10), R384-R387.

Dumpert, K. \& Gnatzy, W. (1977). Cricket combined mechanoreceptors and kicking response. Journal of Comparative Physiology. 122, 9-25.

Eaton J. L. (1988). Lepidopteran anatomy. Xiii + 257 pp. JohnWiley \& Sons, New York. Eberhard, M.J.B., Lang, D., Metscher, B., Pass, G., Picker, M.D. \& Wolf, H. (2010). Structure and sensory physiology of the leg scolopidial organs in Mantophasmatodea and their role in vibrational communication. Arthropod Structure \& Development, 39(4), 230-241.

Fahy, F.J., \& Gardonio, P. (2007). Sound and structural vibration: radiation, transmission and response. Academic press.

Fariñas, M.D., Álvarez-Arenas, T.G., Sancho-Knapik, D., Peguero-Pina, J.J., \& GilPelegrín, E. (2012). Shear waves in plant leaves at ultrasonic frequencies: shear 
properties of vegetal tissues. In Ultrasonics Symposium (IUS), 2012 IEEE International (pp. 1513-1516). IEEE.

Field, L.H., \& Matheson, T. (1998). Chordotonal organs of insects. In Advances in insect physiology (Vol. 27, pp. 1-228). Academic Press.

Fletcher, L.E. (2007). Vibrational signals in a gregarious sawfly larva (Perga affinis): group coordination or competitive signaling? Behavioral Ecology and Sociobiology. 61, 1809-1821.

Fletcher, L.E. (2008). Cooperative signaling as a potential mechanism for cohesion in a gregarious sawfly larva, Perga affinis. Behavioral Ecology and Sociobiology. $62,1127-1138$.

Fletcher, L.E., Yack, J.E., Fitzgerald, T.D. \& Hoy, R.R. (2006). Vibrational communication in the Cherry Leaf Roller caterpillar Caloptilia serotinella (Gracillarioidea: Gracillariidae). Journal of Insect Behaviour. 19, 1-18.

Gnatzy, W. (1976). The ultrastructure of the thread-hairs on the cerci of the cockroach Periplaneta americana L.: The intermoult phase. Journal of Ultrastructure research, 54(1), 124-134.

Griffin, M.J. (2012). Handbook of human vibration. Academic press.

Guedes, R.N.C., Matheson, S.M., Frei, B., Smith, M.L. \& Yack, J.E. (2012). Vibration detection and discrimination in the masked birch caterpillar (Drepana arcuata). Journal of Comparative Physiology A.. 198, 325-335.

Hill, P.S. (2009). How do animals use substrate-borne vibrations as an information source? Naturwissenschaften, 96(12), 1355-1371.

Hinton, H.E. (1946). On the homology and nomenclature of the setae of lepidopterous larvae, with some notes on the phylogeny of the Lepidoptera. Ecological Entomology, 97(1), 1-37.

Hofmann, T., \& Koch, U. T. (1985). Acceleration receptors in the femoral chordotonal organ of the stick insect, Cuniculina impigra. Journal of Experimental Biology, 114(1), 225-237.

Hofmann, T., Koch, U.T., \& Bässler, U. (1985). Physiology of the femoral chordotonal organ in the stick insect, Cuniculina impigra. Journal of Experimental Biology, 114(1), 207-223. 
Howse, P.E. (1964). An investigation into the mode of action of the subgenual organ in the termite, Zootermopsis angusticollis Emerson, and in the cockroach, Periplaneta americana L.. Journal of Insect Physiology. 10, 409424.

Ishay, J., Motro, A., Gitter, S. \& Brown, M.B. (1974). Rhythms in acoustical communication by the oriental Hornet, Vespa orientalis. Animal Behavior. $22,741-744$.

Järvilehto, T., Hämäläinen, H., \& Laurinen, P. (1976). Characteristics of single mechanoreceptive fibres innervating hairy skin of the human hand. Experimental Brain Research, 25(1), 45-61.

Jeram, S., \& Čokl, A. (1996). Mechanoreceptors in insects: Johnston's organ in Nezara viridula (L.) (Pentatomidae, Heteroptera). Pflügers Archiv European Journal of Physiology, 431, R281-R282.

Jones, D., Jones, G., \& Hammock, B. D. (1981). Growth parameters associated with endocrine events in larval Trichoplusia ni (Hübner) and timing of these events with developmental markers. Journal of Insect Physiology, 27(11), 779-788.

Katta, S., Vásquez, V., \& Goodman, M.B. (2018). The dynamics of somatosensory mechanotransduction in $C$. elegans touch receptor neurons. Biophysical Journal, 114(3), 18a-19a.

Kavčič, A., Čokl, A., Laumann, R.A., Blassioli-Moraes, M.C., \& Borges, M. (2013). Tremulatory and abdomen vibration signals enable communication through air in the stink bug Euschistus heros. PLoS One, 8(2), e56503.

Keil, T.A. (1997). Functional morphology of insect mechanoreceptors. Microscopy Research and Technique, 39(6), 506-531.

Keil, T.A., \& Steinbrecht, R. A. (1984). Mechanosensitive and olfactory sensilla of insects. In Insect Ultrastructure (pp. 477-516). Springer, Boston, MA.

Kent, K.S., Fjeld, C.C. \& Anderson, R. (1996). Leg proprioceptors of the tobacco hornworm, Manduca sexta: organization of central projections at larval and adult stages. Microscopy research and technique, 35(3), 265-284.

Kilpinen, O. \& Storm, J. (1997). Biophysics of the subgenual organ of the honeybee, Apis mellifera. Journal of Comparative Physiology A. 181, 309-318. 
Kočárek, P. (2009). Sound production and chorusing behaviour in larvae of Icosium tomentosum. Central European Journal of Biology. 4, 422-426.

Kojima, W., Ishikawa, Y., \& Takanashi, T. (2012a). Pupal vibratory signals of a groupliving beetle that deter larvae: are they mimics of predator cues?. Communicative \& integrative Biology, 5(3), 262-264.

Kojima, W., Takanashi, T., \& Ishikawa, Y. (2012b). Vibratory communication in the soil: pupal signals deter larval intrusion in a group-living beetle Trypoxylus dichotoma. Behavioral Ecology and Sociobiology, 66(2), 171-179.

Konietzny, F., \& Hensel, H. (1977). Response of rapidly and slowly adapting mechanoreceptors and vibratory sensitivity in human hairy skin. Pflügers Archiv, 368(1-2), 39-44.

Kühne, R. (1982). Neurophysiology of the vibration sense in locusts and bushcrickets: response characteristics of single receptor units. Journal of Insect Physiology. 28, 155-157, 159-163.

Lewis, F.P. and Fullard, J.H. (1996). Neurometamorphosis of the ear in the gypsy moth, Lymantria dispar, and its homologue in the earless forest tent caterpillar moth, Malacosoma disstria. Developmental Neurobiology, 31(2), 245-262.

Lumpkin, E.A., Marshall, K.L., \& Nelson, A.M. (2010). The cell biology of touch. The Journal of Cell Biology, 191(2), 237-248.

Magal, C., Schöller, M., Tautz, J., \& Casas, J. (2000). The role of leaf structure in vibration propagation. Journal of the Acoustical Society of America, 108(5), 2412-2418.Griffin, M. J. (2012). Handbook of human vibration. Academic press.

Mahns, D.A., Perkins, N. M., Sahai, V., Robinson, L., \& Rowe, M.J. (2006). Vibrotactile frequency discrimination in human hairy skin. Journal of Neurophysiology, 95(3), 1442-1450.

Markl, H. \& Tautz, J. (1975). The sensitivity of hair receptors in caterpillars of Barathra brassicae L. (Lepidoptera, Noctuidae) to particle movement in a sound field. Journal of Comparative Physiology. 99, 79-87.

Martens, M.J., \& Michelsen, A. (1981). Absorption of acoustic energy by plant leaves. Journal of the Acoustical Society of America, 69(1), 303-306. 
Michelsen, A. (1979). Insect ears as mechanical systems: Recent study has revealed that insect ears use a variety of ingenious mechanisms to determine the direction from which sound comes and to analyze its frequency. American Scientist, 67(6), 696-706.

Michelsen, A., \& Nocke, H. (1974). Biophysical aspects of sound communication in insects. In Advances in insect physiology (Vol. 10, pp. 247-296). Academic Press.

Montealegre-z, F., Jonsson, T., Robson-Brown, K.A., Postles, M., \& Robert, D. (2012). Convergent evolution between insect and mammalian audition. Science, 338(6109), 968-971.

Narins, P. M., \& Lewis, E. R. (1984). The vertebrate ear as an exquisite seismic sensor. Journal of the Acoustical Society of America, 76(5), 1384-1387.

Nesterov, A., Spalthoff, C., Kandasamy, R., Katana, R., Rankl, N. B., Andrés, M., ... \& Warren, B. (2015). TRP channels in insect stretch receptors as insecticide targets. Neuron, 86(3), 665-671.

O'Neil, R.G., \& Heller, S. (2005). The mechanosensitive nature of TRPV channels. Pflügers Archiv, 451(1), 193-203.

Peterson, B.A., \& Weeks, J.C. (1988). Somatotopic mapping of sensory neurons innervating mechanosensory hairs on the larval prolegs of Manduca sexta. Journal of Comparative Neurology, 275(1), 128-144.

Platt, N., \& Reynolds, S. E. (1986). The pharmacology of the heart of a caterpillar, the tobacco hornworm, Manduca sexta. Journal of Insect Physiology, 32(3), 221230.

R Core Team (2016). R: A language and environment for statistical computing. R foundation for statistical computing, Vienna, Austria. http://www.Rproject.org/..

Rota, J., \& Wagner, D.L. (2008). Wormholes, sensory nets and hypertrophied tactile setae: the extraordinary defence strategies of Brenthia caterpillars. Animal Behaviour, 76(5), 1709-1713. 
Savoyard, J.L., Gamboa, G.J., Cummings, D.L.D. \& Foster, R.L. (1998). The communicative meaning of body oscillations in the social wasp, Polistes fuscatus (Hymenoptera, Vespidae). Insectes Sociaux. 45, 215-230.

Scott, J.L. \& Yack, J.E. (2012). Vibratory territorial signals in caterpillars of the poplar lutestring, Tethea or (Lepidoptera: Drepanidae). European Journal of Entomology. 109, 411-417.

Scott, J.L., Kawahara, A.Y., Skevington, J.H., Yen, S., Sami A., Smith M.L. \& Yack, J.E. (2010). The evolutionary origins of ritualized acoustic signals in caterpillars. Nature Communications. 1, 4.

Scrucca, L. (2011) Model-based \{SIR\} for dimension reduction. Computational Statistics \& Data Analysis, 5(11), 3010-3026.

Shaw, S. (1994a). Detection of airborne sound by a cockroach 'vibration detector': a possible missing link in insect auditory evolution. Journal of Experimental Biology, 193(1), 13-47.

Shaw, S.R. (1994b). Re-evaluation of the absolute threshold and response mode of the most sensitive know "vibration" detector, the cockroach's subgenual organ: A cochlea-like displacement threshold and a direct response to sound. Journal of Neurobiology. 25, 1167-1185.

Shimozawa, T., \& Kanou, M. (1984). Varieties of filiform hairs: range fractionation by sensory afferents and cereal interneurons of a cricket. Journal of Comparative Physiology A: Neuroethology, Sensory, Neural, and Behavioral Physiology, 155(4), 485-493.

Simon, M.A., \& Trimmer, B.A. (2009). Movement encoding by a stretch receptor in the soft-bodied caterpillar, Manduca sexta. Journal of Experimental Biology, 212(7), 1021-1031.

Stehr, F. W. (1987). Order Lepidoptera. Immature insects, 288-305.

Strauß, J., \& Lakes-Harlan, R. (2017). Vibrational sensitivity of the subgenual organ complex in female Sipyloidea sipylus stick insects in different experimental paradigms of stimulus direction, leg attachment, and ablation of a connective tibial sense organ. Comparative Biochemistry and Physiology Part A: Molecular \& Integrative Physiology, 203, 100-108. 
Tamarkin, D.A., and Levine, R.B. (1996). Synaptic interactions between a muscleassociated proprioceptor and body wall muscle motor neurons in larval and adult Manduca sexta. Journal of Neurophysiology, 76(3), 1597-1610.

Tang, S.H., Ong, P.P., \& Woon, H.S. (1986). Monte Carlo simulation of sound propagation through leafy foliage using experimentally obtained leaf resonance parameters. Journal of the Acoustical Society of America, 80(6), 1740-1744.

Tautz, J. \& Markl, H. (1978). Caterpillars detect flying wasps by hairs sensitive to airborne vibration. Behavioral Ecology and Sociobiology. 4, 101-110.

Tautz, J. (1977). Reception of medium vibration by thoracal hairs of caterpillars of Barathra brassicae L. (Lepidoptera, Noctuidae). I. Mechanical Properties of the Receptor Hairs. Journal of Comparative Physiology. 118, 13-31.

Tautz, J. (1978). Reception of medium vibration by thoracal hairs of caterpillars of Barathra brassicae L. (Lepidoptera, Noctuidae). II. Response Characteristics of the Sensory Cell. Journal of Comparative Physiology. 118, 13-31.

Ter Hofstede, H.M., Goerlitz, H.R., Montealegre-Z, F., Robert, D., \& Holderied, M.W. (2011). Tympanal mechanics and neural responses in the ears of a noctuid moth. Naturwissenschaften, 98(12), 1057-1061.

Thurm, U. (1965). An insect mechanoreceptor part I: Fine structure and adequate stimulus. In Cold Spring Harbor Symposia on Quantitative Biology (Vol. 30, pp. 75-82). Cold Spring Harbor Laboratory Press.

Todd, N.P.M., Rosengren, S.M., \& Colebatch, J.G. (2008). Tuning and sensitivity of the human vestibular system to low-frequency vibration. Neuroscience letters, 444(1), 36-41.

van Griethuijsen, L.I., and Trimmer, B.A. (2010). Caterpillar crawling over irregular terrain: anticipation and local sensing. Journal of Comparative Physiology A, 196(6), 397-406.

Vedel, J.P., \& Roll, J.P. (1982). Response to pressure and vibration of slowly adapting cutaneous mechanoreceptors in the human foot. Neuroscience Letters, 34(3), 289-294.

Wittmann, M.C., Steinberg, R. N., \& Redish, E. F. (2002). Making sense of how students make sense of mechanical waves. arXiv preprint physics/0207092. 
Yack, J. (2016). Vibrational signaling. In Insect Hearing (pp. 99-123). Springer International Publishing.

Yack, J.E. (2004). The structure and function of auditory chordotonal organs in insects. Microscopy Research and Technique. 63, 315-337.

Yack, J.E., Gill, S., Drummond-Main, C., and Sherratt, T.N. (2014). Residency duration and shelter quality influence vibratory signalling displays in a territorial caterpillar. Ethology, 120(4), 354-364.

Yack, J.E., Smith, M.L., and Weatherhead, P.J. (2001). Caterpillar talk: Acoustically mediated territoriality in larval Lepidoptera. Proceedings of the National Academy of Sciences. 98, 11371-11375.

Yadav, C., Guedes, R.N.C., Matheson, S.M., Timbers, T.A., and Yack, J.E. (2017). Invitation by vibration: recruitment to feeding shelters in social caterpillars. Behavioral Ecology and Sociobiology, 71(3), 51.

Young, D. (1970). The structure and function of a connective chordotonal organ in the cockroach leg. Philosophical Transactions of the Royal Society. 256, 401-426.

Zacharuk, R.Y. \& Shields, V.D. (1991). Sensilla of immature insects. Annual Review of Entomology, 36(1), 331-354. 


\section{Appendices}

\section{Appendix A (Supplements to Chapter 2)}

\section{Appendix A.1 - Tables with samples of data.}

Thresholds - Sample of data used to calculate thresholds with Local Regression and magnitude of response. Each row corresponds to one stimulus signal and the number of spikes detected before and during it. The complete spreadsheet contains 8,322 rows with 8 caterpillars, 8 frequencies, 21 stimulus intensities for each frequency and 4 to 10 replicates of each combination of the previous parameters. The results obtained from the analysis of data in this and the other tables are found in the Results section.

\begin{tabular}{cccccccccc}
\hline $\begin{array}{c}\text { Frequency } \\
(\mathbf{H z})\end{array}$ & $\begin{array}{c}\text { Caterpillar } \\
\text { ID }\end{array}$ & $\begin{array}{c}\text { Stimulus } \\
\text { ID }\end{array}$ & $\begin{array}{c}\text { Displacement } \\
\text { (um) }\end{array}$ & $\begin{array}{c}\text { Velocity } \\
\text { (mm/s) }\end{array}$ & $\begin{array}{c}\text { Acceleration } \\
\text { (m/s2) }\end{array}$ & $\begin{array}{c}\# \\
\text { Spikes } \\
\text { Before }\end{array}$ & $\begin{array}{c}\# \\
\text { Spikes } \\
\text { During }\end{array}$ & $\begin{array}{c}\text { \# Difference } \\
\text { (Dur. - Bef.) }\end{array}$ \\
\hline 10 & 3 & 2 & 1.06 & 0.09 & 0.14 & 11 & 14 & 3 \\
10 & 3 & 3 & 1.43 & 0.10 & 0.17 & 13 & 12 & -1 \\
10 & 3 & 20 & 48.15 & 1.41 & 0.24 & 10 & 18 & 8 & 11 \\
10 & 3 & 21 & 67.56 & 2.12 & 0.29 & 8 & 19 & 4 \\
80 & 4 & 2 & 0.89 & 0.08 & 0.15 & 12 & 16 & -2 \\
80 & 4 & 3 & 0.97 & 0.13 & 0.15 & 14 & 12 & 38 \\
80 & 4 & 20 & 44.51 & 11.10 & 5.70 & 10 & 48 & 35 \\
80 & 4 & 21 & 62.91 & 15.79 & 8.06 & 12 & 47 & 35 \\
\hline
\end{tabular}


Directional - Sample of data used to compare the hair sensitivity in different directions. These data came from recordings of different caterpillars than those in tables with sample of data for Thresholds, Phase-locking in all caterpillars and Phase locking in one caterpillar. Each row corresponds to one step or sine stimulus signal. The complete spreadsheet contains 3449 rows, with 4 caterpillars and 6 orientations (anterior, posterior, medial and lateral for step stimuli; antero-posterior and latero-medial for sine stimuli).

\begin{tabular}{|c|c|c|c|c|c|c|c|c|}
\hline Orientation & $\begin{array}{c}\text { Caterpillar } \\
\text { ID }\end{array}$ & $\begin{array}{l}\text { Stimulus } \\
\text { ID }\end{array}$ & $\begin{array}{l}\text { Displacement } \\
\quad \text { (um) }\end{array}$ & $\begin{array}{l}\text { Velocity } \\
(\mathrm{mm} / \mathrm{s})\end{array}$ & $\begin{array}{l}\text { Acceleration } \\
(\mathrm{m} / \mathrm{s} 2)\end{array}$ & $\begin{array}{c}\# \\
\text { Spikes } \\
\text { Before } \\
\end{array}$ & $\begin{array}{c}\# \\
\text { Spikes } \\
\text { During } \\
\end{array}$ & $\begin{array}{l}\text { \# Difference } \\
\text { (Dur. - Bef.) }\end{array}$ \\
\hline Anterior & 19 & 20 & 15.51 & 0.66 & 0.17 & 11 & 7 & -4 \\
\hline Anterior & 19 & 21 & 23.75 & 0.96 & 0.20 & 7 & 11 & 4 \\
\hline Posterior & 20 & 20 & 15.04 & 0.68 & 0.20 & 7 & 7 & 0 \\
\hline Posterior & 20 & 21 & 22.56 & 1.06 & 0.24 & 3 & 6 & 3 \\
\hline Medial & 20 & 20 & 15.20 & 0.67 & 0.19 & 8 & 9 & 1 \\
\hline Medial & 20 & 21 & 23.88 & 0.94 & 0.20 & 5 & 9 & 4 \\
\hline Lateral & 20 & 20 & 15.20 & 0.67 & 0.19 & 8 & 7 & -1 \\
\hline Lateral & 20 & 21 & 23.88 & 0.94 & 0.20 & 5 & 9 & 4 \\
\hline $\begin{array}{l}\text { Antero- } \\
\text { posterior }\end{array}$ & 16 & 20 & 44.78 & 11.30 & 5.80 & 13 & 67 & 54 \\
\hline $\begin{array}{l}\text { Antero- } \\
\text { posterior }\end{array}$ & 16 & 21 & 63.67 & 16.03 & 8.20 & 4 & 45 & 41 \\
\hline $\begin{array}{l}\text { Latero- } \\
\text { medial }\end{array}$ & 16 & 20 & 42.84 & 10.70 & 5.70 & 18 & 72 & 54 \\
\hline $\begin{array}{l}\text { Latero- } \\
\text { medial }\end{array}$ & 16 & 21 & 60.82 & 15.18 & 7.97 & 16 & 85 & 69 \\
\hline$\ldots$ & $\ldots$ & $\ldots$ & $\ldots$ & $\ldots$ & $\ldots$ & $\ldots$ & $\ldots$ & $\ldots$ \\
\hline
\end{tabular}


Phase-locking in all caterpillars - Sample of data used in the phase locking analysis for frequencies from 10 to $905 \mathrm{~Hz}$. They came from the same recordings as those in the table with sample of data for Thresholds, although here only supra-threshold stimulus signals were used, i.e. signals with higher peak velocities than $1.0 \mathrm{~mm} / \mathrm{s}$. Each row corresponds to one spike and its relative phase. The complete spreadsheet contains 81,914 rows with 8 caterpillars, 8 frequencies and varying number of stimulus signals.

\begin{tabular}{cccccccccc}
\hline $\begin{array}{c}\text { Frequency } \\
\text { (Hz) }\end{array}$ & $\begin{array}{c}\text { Caterpillar } \\
\text { ID }\end{array}$ & $\begin{array}{c}\text { Stimulus } \\
\text { ID }\end{array}$ & $\begin{array}{c}\text { Wave } \\
\text { ID }\end{array}$ & $\begin{array}{c}\text { Spike } \\
\text { ID }\end{array}$ & $\begin{array}{c}\text { Displacement } \\
\text { (um) }\end{array}$ & $\begin{array}{c}\text { Velocity } \\
\text { (mm/s) }\end{array}$ & $\begin{array}{c}\text { Acceleration } \\
\text { (m/s2) }\end{array}$ & $\begin{array}{c}\text { Relative } \\
\text { angle } \\
\text { (rads) }\end{array}$ & $\begin{array}{c}\text { Relative } \\
\text { time } \\
\text { (ms) }\end{array}$ \\
\hline 10 & 2 & 20 & 1 & 1 & 46.03 & 1.44 & 0.29 & 0.31 & 5.12 \\
10 & 2 & 20 & 1 & 2 & 46.03 & 1.44 & 0.29 & 0.92 & 15.07 \\
10 & 2 & 20 & 1 & 3 & 46.03 & 1.44 & 0.29 & 1.25 & 20.36 \\
10 & 2 & 20 & 1 & 4 & 46.03 & 1.44 & 0.29 & 1.71 & 27.89 \\
80 & 3 & 77 & 1 & 1 & 5.95 & 1.42 & 0.81 & 3.69 & 7.21 \\
80 & 3 & 77 & 1 & 2 & 5.95 & 1.42 & 0.81 & 5.84 & 11.43 \\
80 & 3 & 77 & 2 & 1 & 5.95 & 1.42 & 0.81 & 2.95 & 5.65 \\
80 & 3 & 77 & 3 & 1 & 5.95 & 1.42 & 0.81 & 0.93 & 1.88 \\
$\ldots$ & $\ldots$ & $\ldots$ & $\ldots$ & $\ldots$ & $\ldots$ & $\ldots$ & $\ldots$ & $\ldots$ & $\ldots$ \\
\hline
\end{tabular}


Phase locking in one caterpillar - Sample of data used in the phase locking analysis for frequencies from 10 to $120 \mathrm{~Hz}$. Similarly to the data in the table with sample of data for Phase-locking in all caterpillars, data in this table were also used to generate rose histograms. However the signals here have more repetitions - at least 50 times for each combination of frequency and amplitude - while the signals in the previous table have about 5 repetitions. The larger number of repetitions in this table was only possible due to the quality of caterpillar \#7, whose nerve was alive for longer even after de-efferenting. The complete spreadsheet contains 14,933 rows with 10 frequencies but only 1 caterpillar.

\begin{tabular}{ccccccccccc}
\hline $\begin{array}{c}\text { Frequency } \\
\text { (Hz) }\end{array}$ & $\begin{array}{c}\text { Caterpillar } \\
\text { ID }\end{array}$ & $\begin{array}{c}\text { Stimulus } \\
\text { ID }\end{array}$ & $\begin{array}{c}\text { Wave } \\
\text { ID }\end{array}$ & $\begin{array}{c}\text { Spike } \\
\text { ID }\end{array}$ & $\begin{array}{c}\text { Displacement } \\
\text { (um) }\end{array}$ & $\begin{array}{c}\text { Velocity } \\
\text { (mm/s) }\end{array}$ & $\begin{array}{c}\text { Acceleration } \\
\text { (m/s2) }\end{array}$ & $\begin{array}{c}\text { Relative } \\
\text { angle } \\
\text { (rads) }\end{array}$ & $\begin{array}{c}\text { Relative } \\
\text { time } \\
\text { (ms) }\end{array}$ \\
\hline 90 & 7 & 21 & 6 & 1 & 42.46 & 12.04 & 6.89 & 1.48 & 2.62 \\
90 & 7 & 21 & 6 & 2 & 42.46 & 12.04 & 6.89 & 5.31 & 9.38 \\
100 & 7 & 2 & 10 & 2 & 32.44 & 10.07 & 6.52 & 5.98 & 9.54 \\
100 & 7 & 2 & 11 & 1 & 32.44 & 10.07 & 6.52 & 2.96 & 4.71 \\
110 & 7 & 23 & 19 & 1 & 23.77 & 8.15 & 5.72 & 1.33 & 1.93 \\
110 & 7 & 23 & 19 & 2 & 23.77 & 8.15 & 5.72 & 4.84 & 7.00 \\
120 & 7 & 9 & 20 & 3 & 18.87 & 7.12 & 5.45 & 5.57 & 7.33 \\
120 & 7 & 9 & 21 & 1 & 18.87 & 7.12 & 5.45 & 2.40 & 3.19 \\
$\ldots$ & $\ldots$ & $\ldots$ & $\ldots$ & $\ldots$ & $\ldots$ & $\ldots$ & $\ldots$ & $\ldots$ & $\ldots$ \\
\hline
\end{tabular}


Appendix A.2 - Local regressions loess() for all frequencies in all $T$. $n i$ caterpillars

Local regressions loess() for all frequencies in all T. $n i$ caterpillars. Each subplot shows data from one caterpillar, whose ID is indicated by the number label on its top left. Units on the horizontal axes are Peak velocity $(\mathrm{mm} / \mathrm{s})$ and on the vertical axes are difference in \# spikes (i.e. \# spikes during minus \# spikes before a stimulus). Each data point represents one stimulus signal. Black, dark grey and light grey lines represent the fit, $99 \%$ confidence intervals and $95 \%$ predict intervals of the local regression, respectively. The dashed line at each subplot indicates the point at which the lower confidence interval surpassed the zero line for the first time, which was defined as the threshold for that specific caterpillar to that frequency. 


\section{Frequency: $10 \mathrm{~Hz}$}
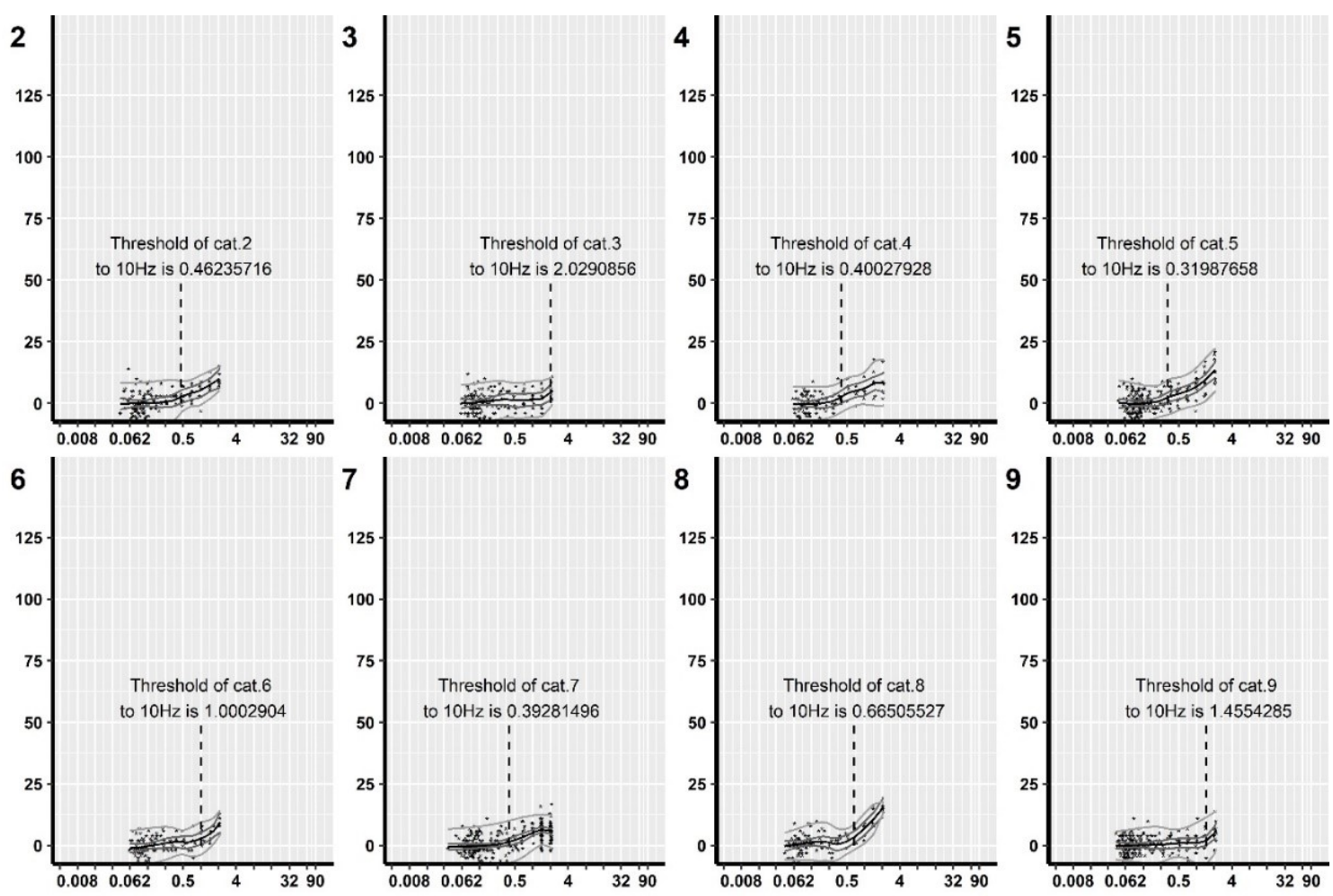

Frequency: $20 \mathrm{~Hz}$
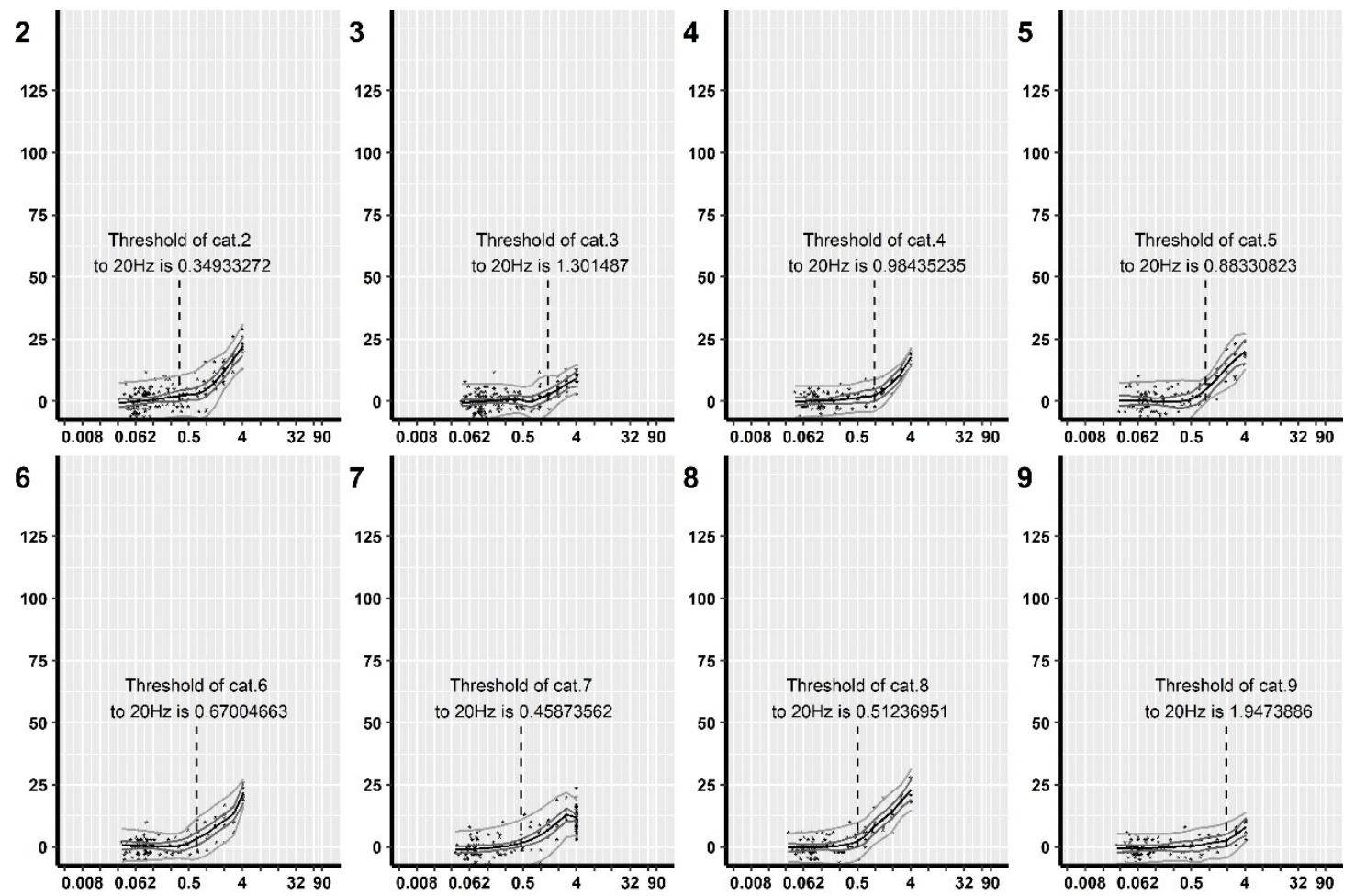


\section{Frequency: $40 \mathrm{~Hz}$}
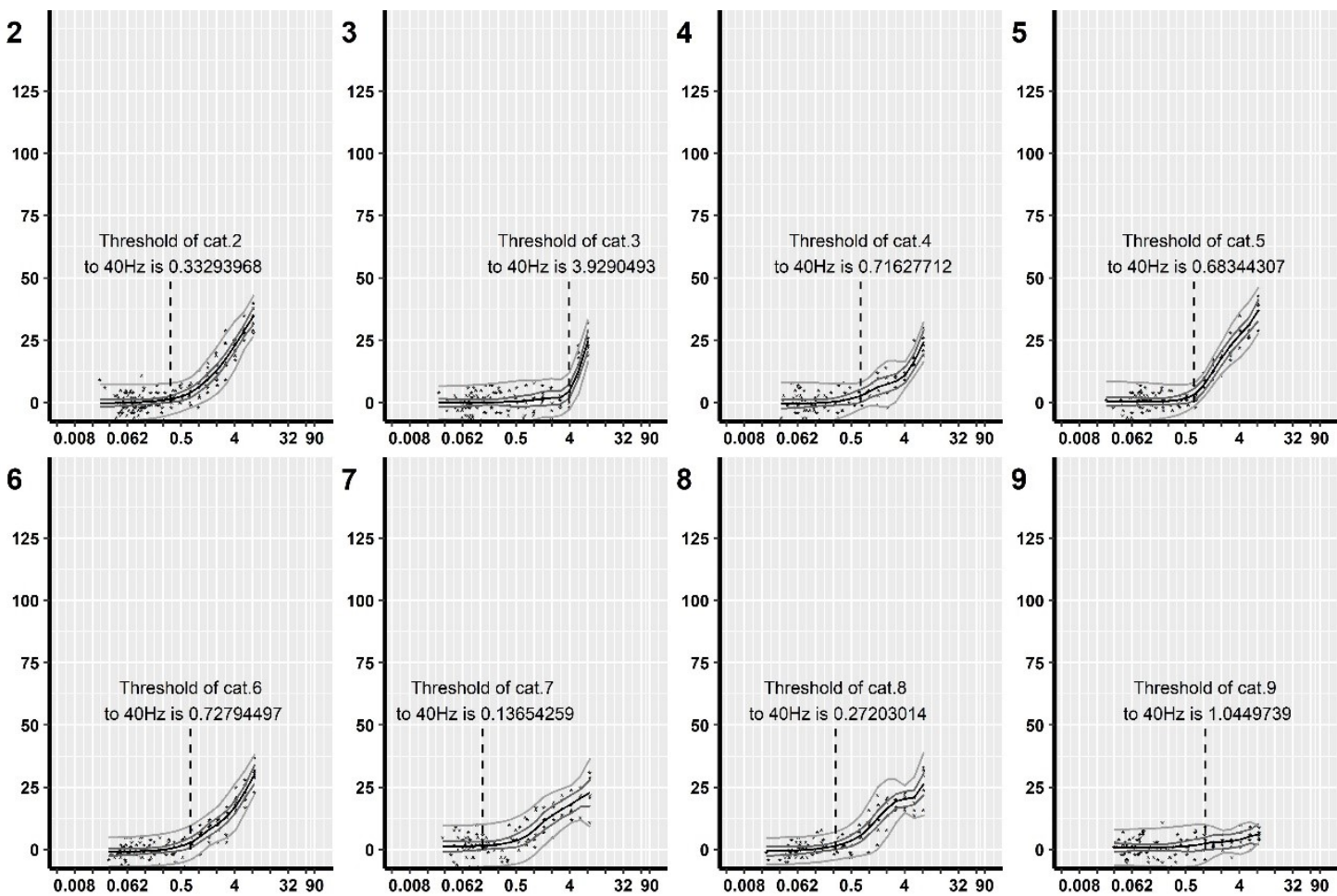

Frequency: $80 \mathrm{~Hz}$
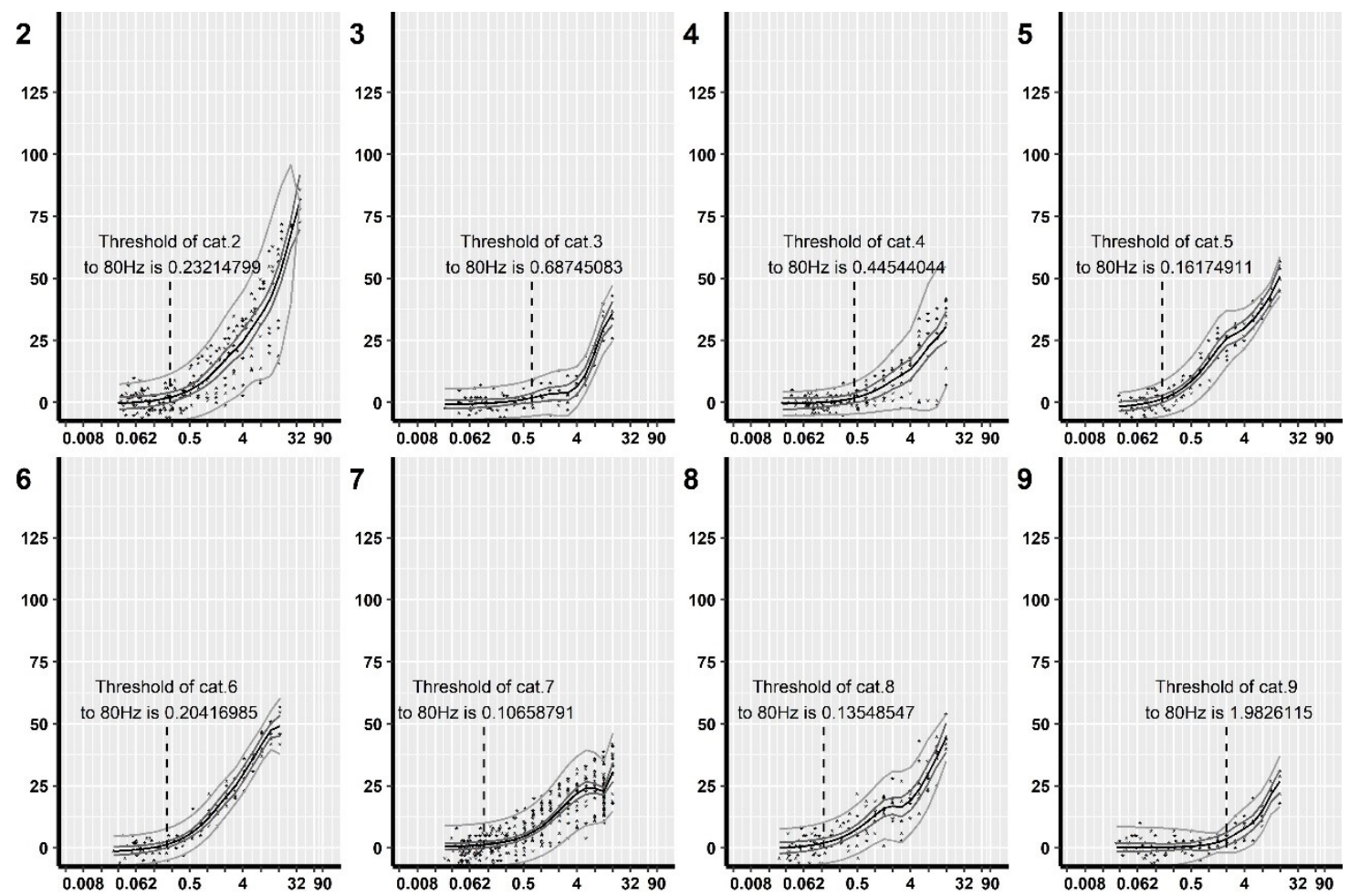


\section{Frequency: $160 \mathrm{~Hz}$}
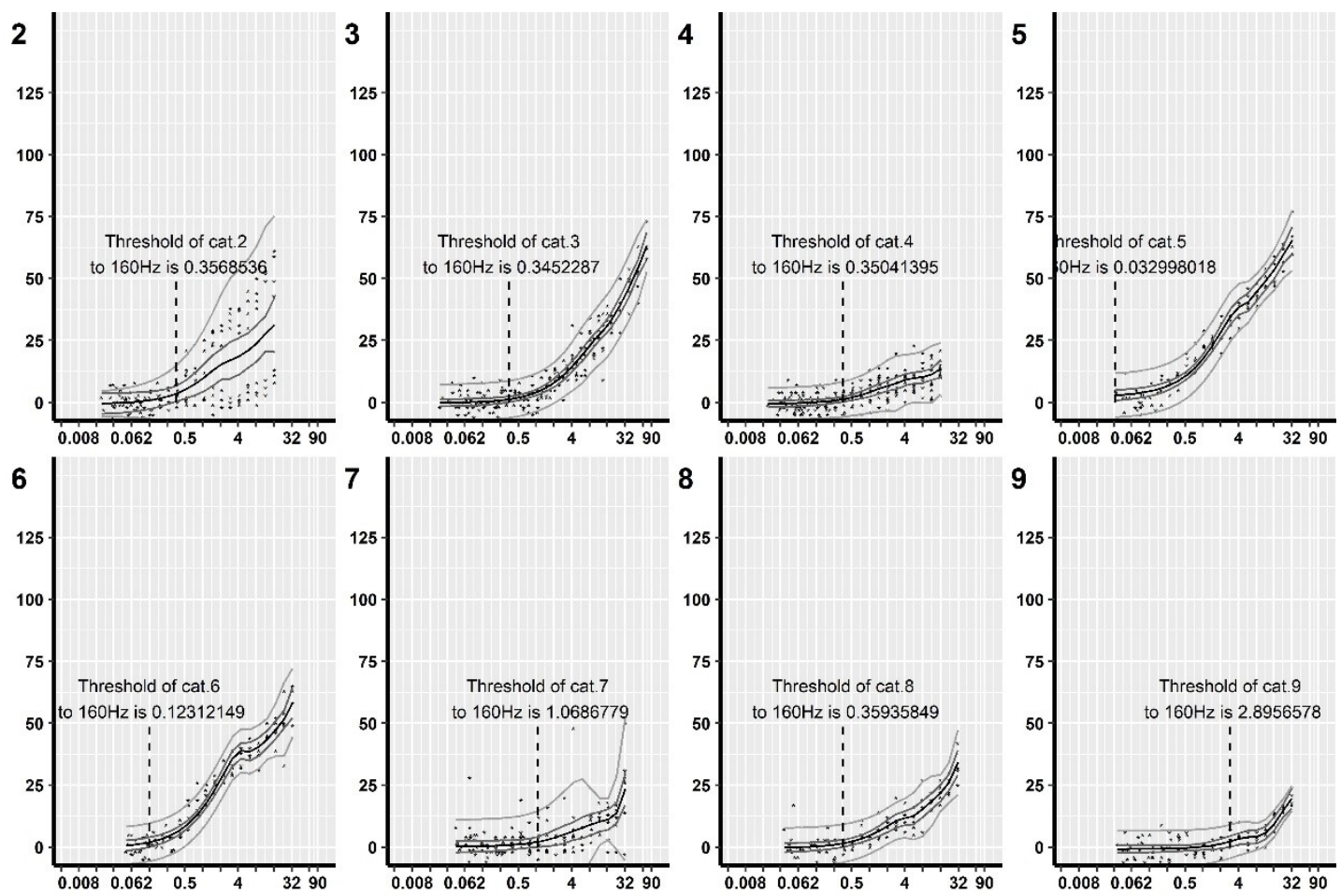

Frequency: $320 \mathrm{~Hz}$
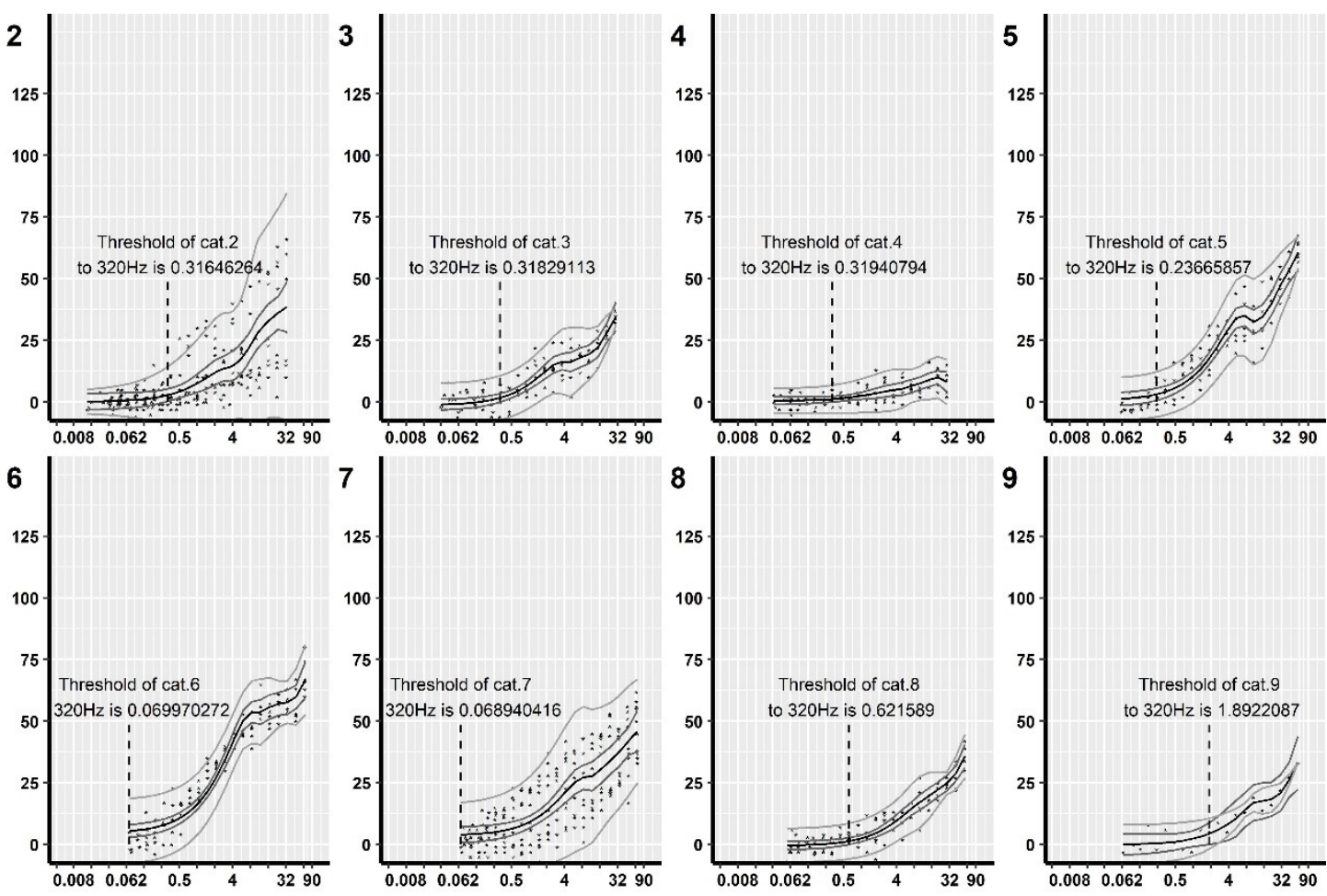


\section{Frequency: $640 \mathrm{~Hz}$}
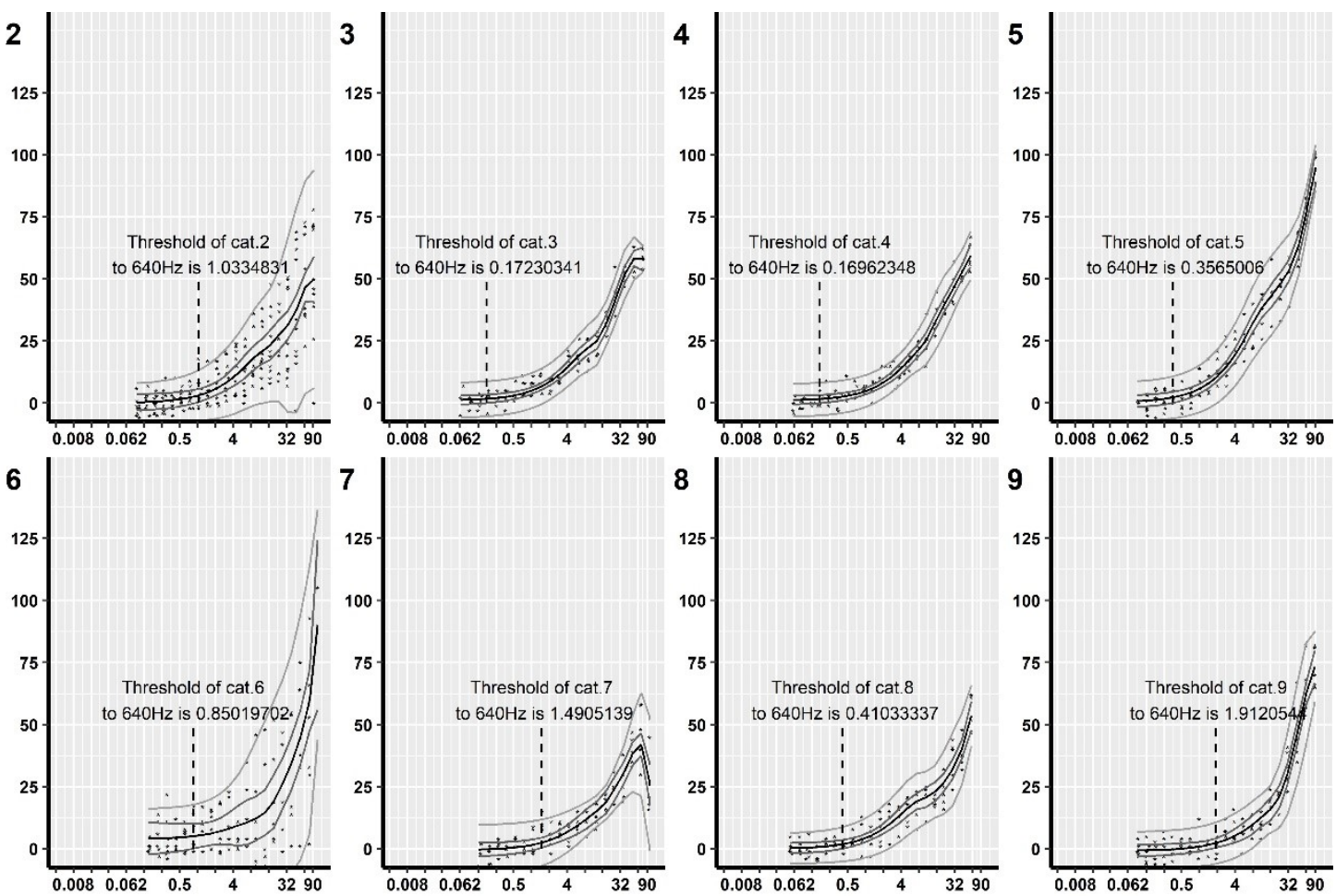

\section{Frequency: $905 \mathrm{~Hz}$}

2

3
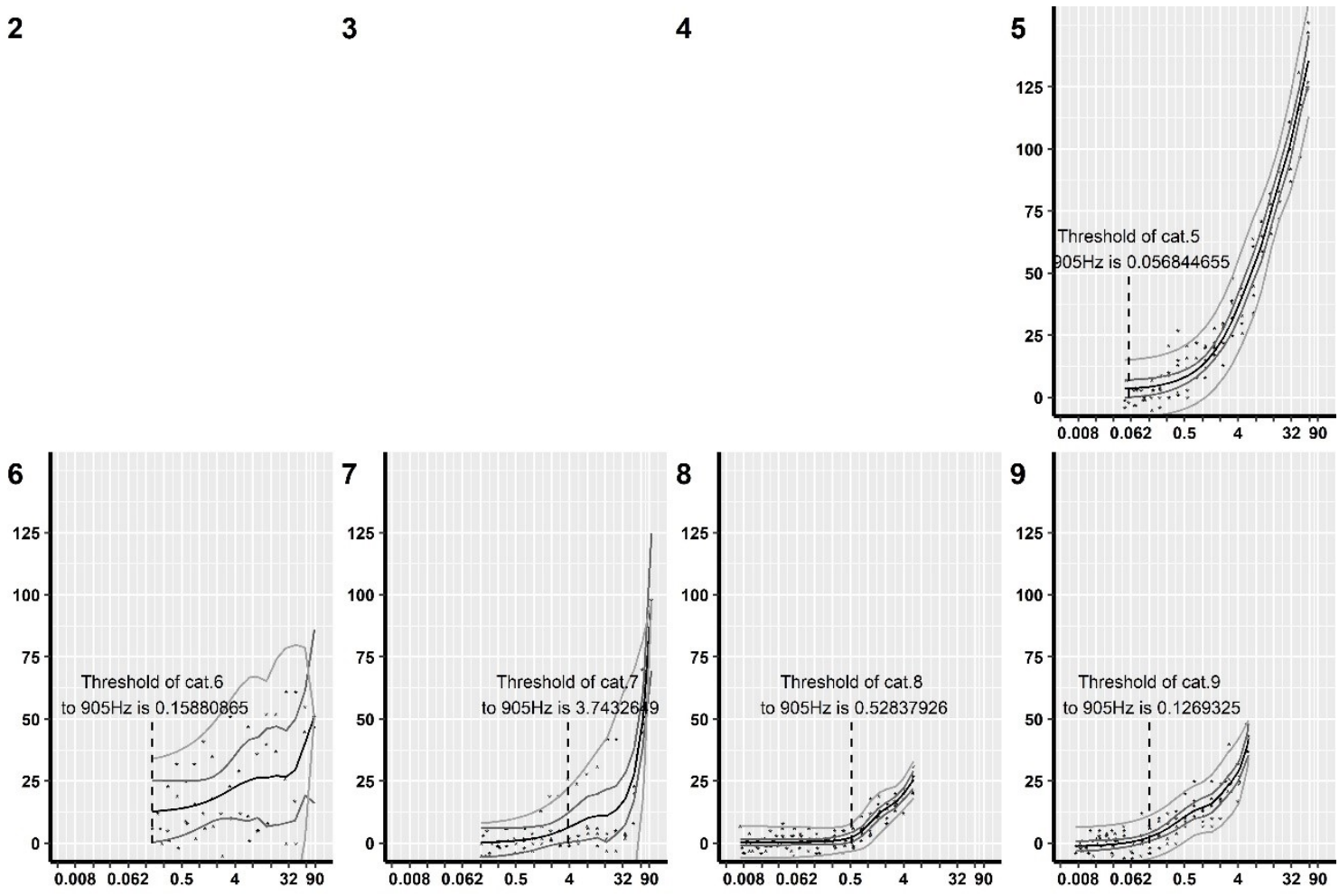
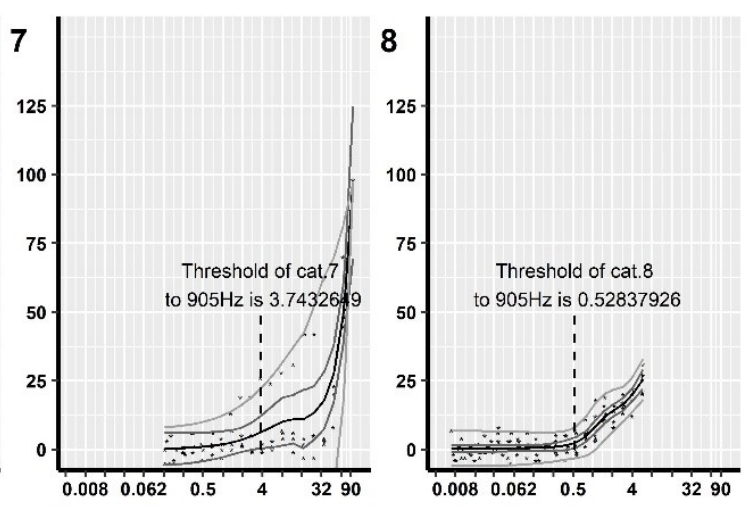

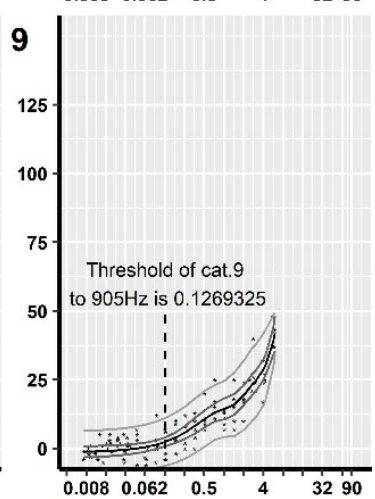


Appendix A.3 - Sample traces of neural activity from proleg hair in $T . n i$

Sample traces of neural activity from proleg hair in $T . n i$ responding to sine stimuli of different frequencies and intensities. All traces were extracted from recordings of caterpillar \#8, whose Ventral Nerve had been de-efferented since the beginning of the experiment. Therefore, any potential motor activity had been silenced and all neural activity that is visible on the traces is sensory. 


\section{Frequency of $10 \mathrm{~Hz}$ :}
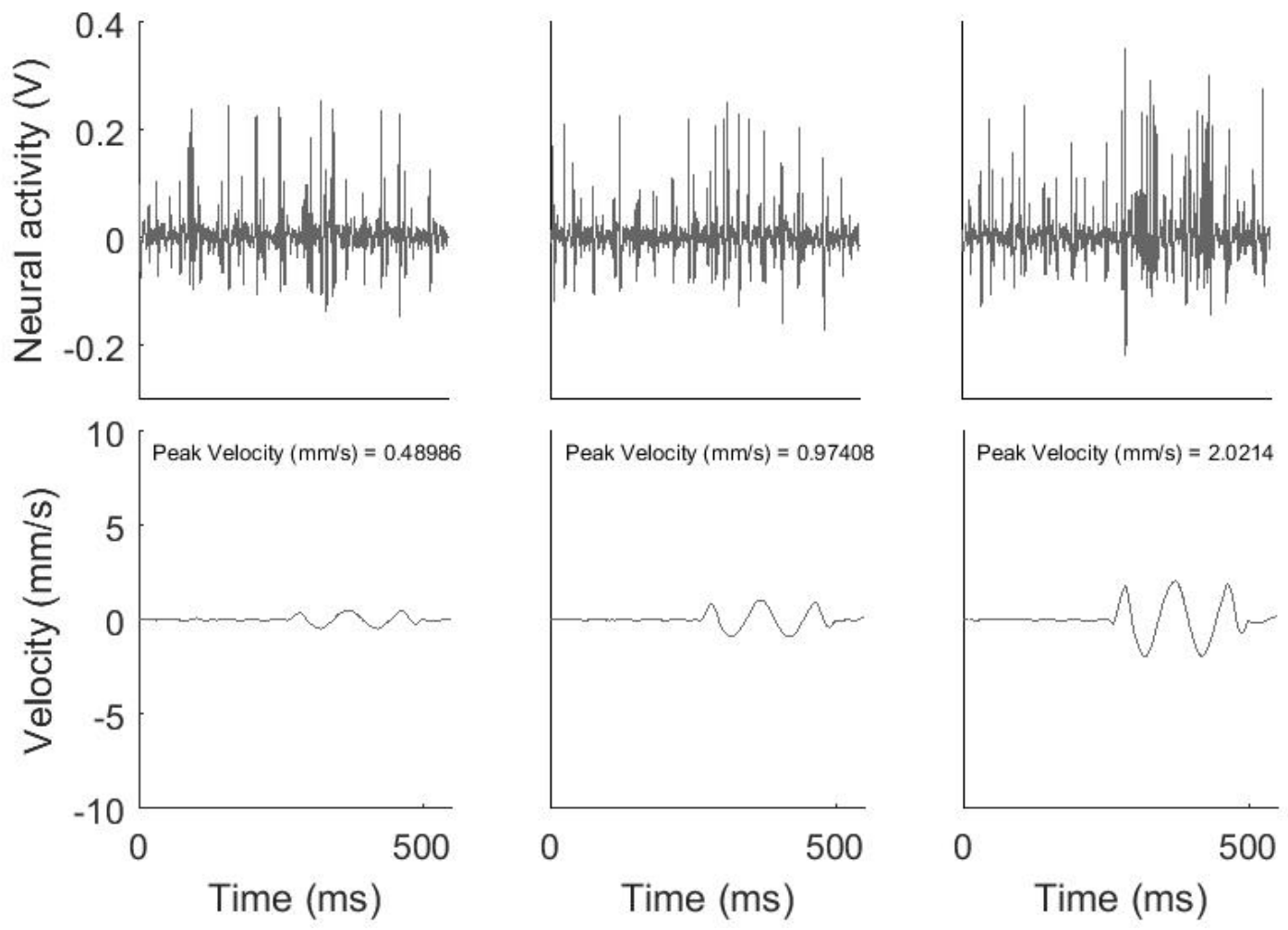

Frequency of $20 \mathrm{~Hz}$ :
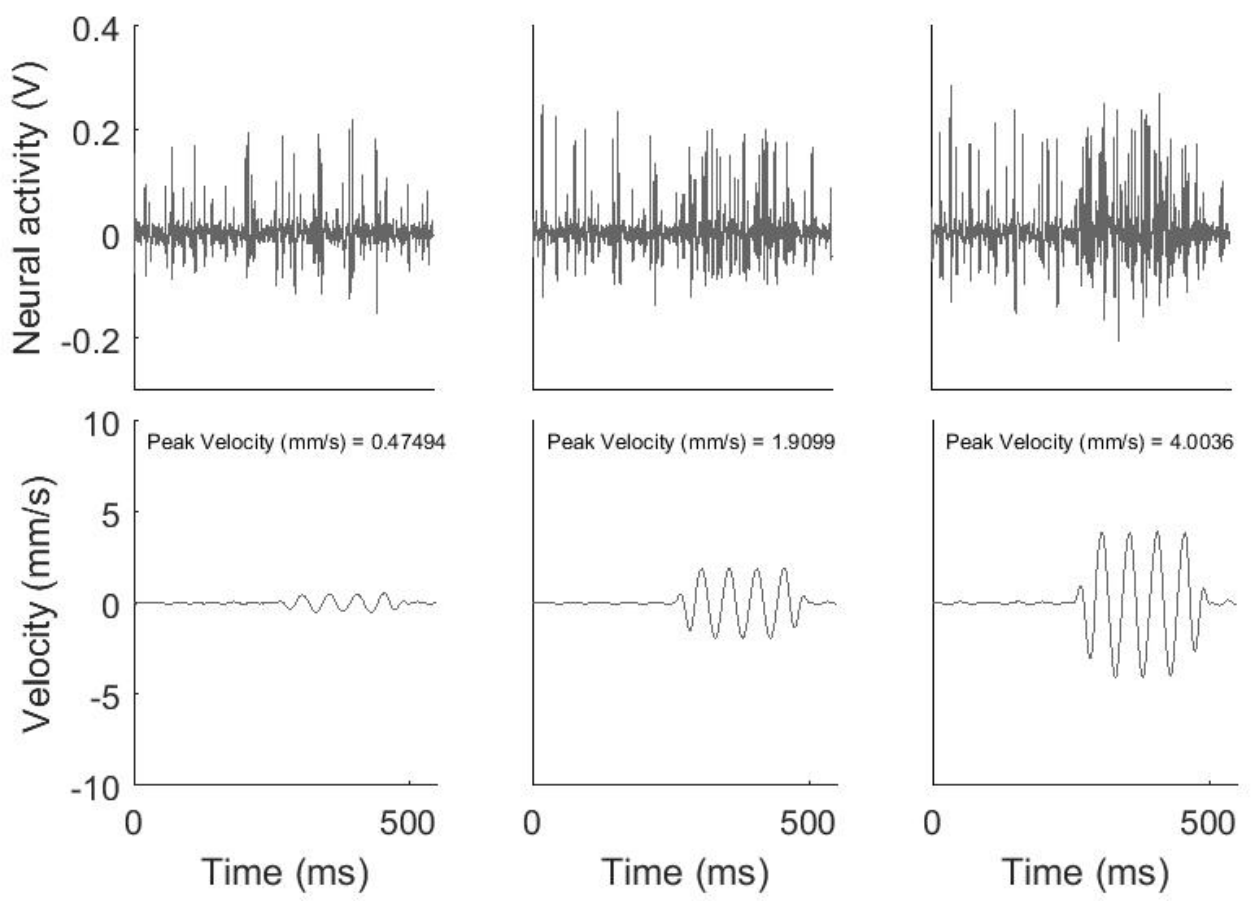


\section{Frequency of $40 \mathrm{~Hz}$ :}
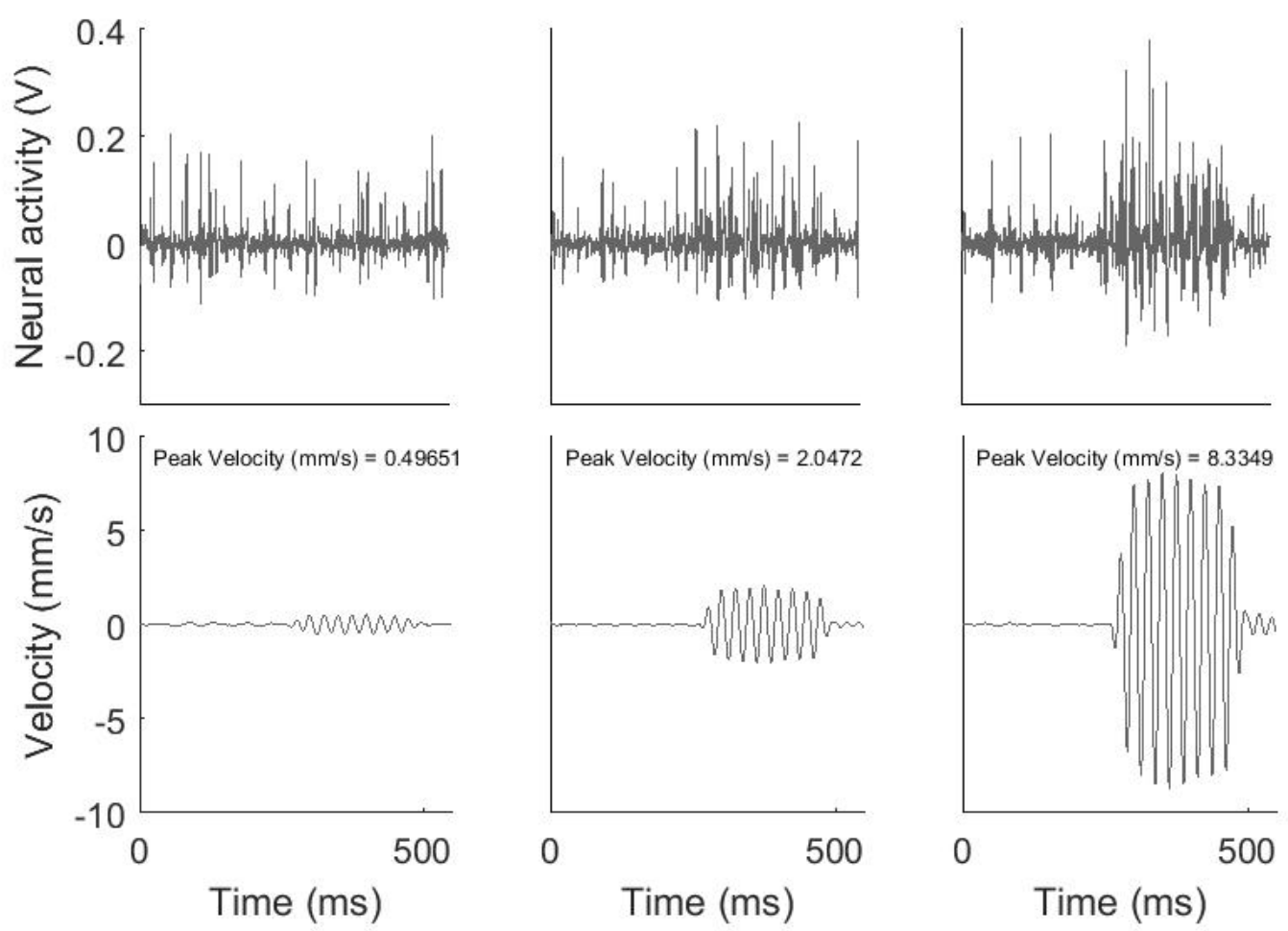

Frequency of $80 \mathrm{~Hz}$ :
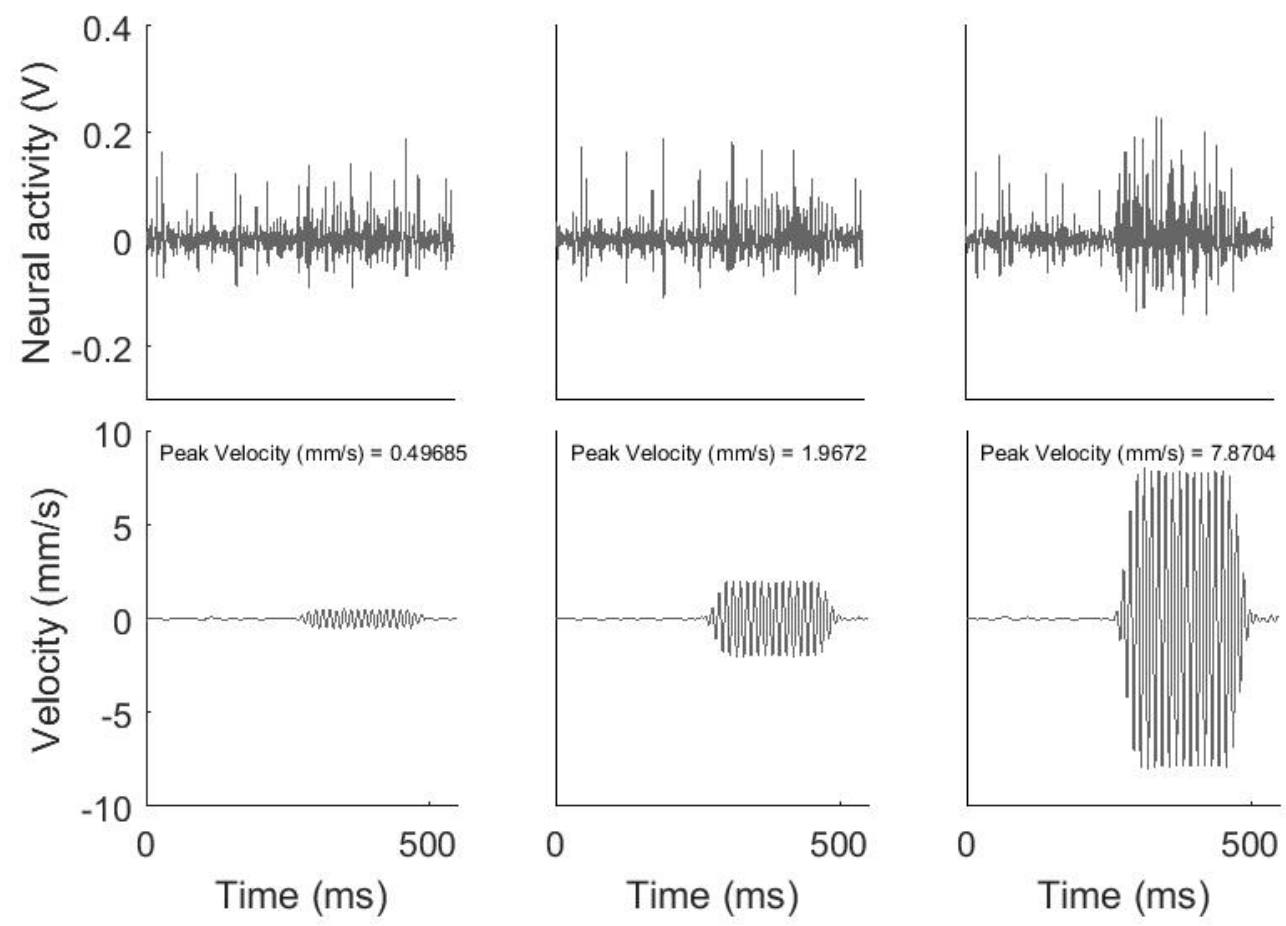


\section{Frequency of $160 \mathrm{~Hz}$ :}
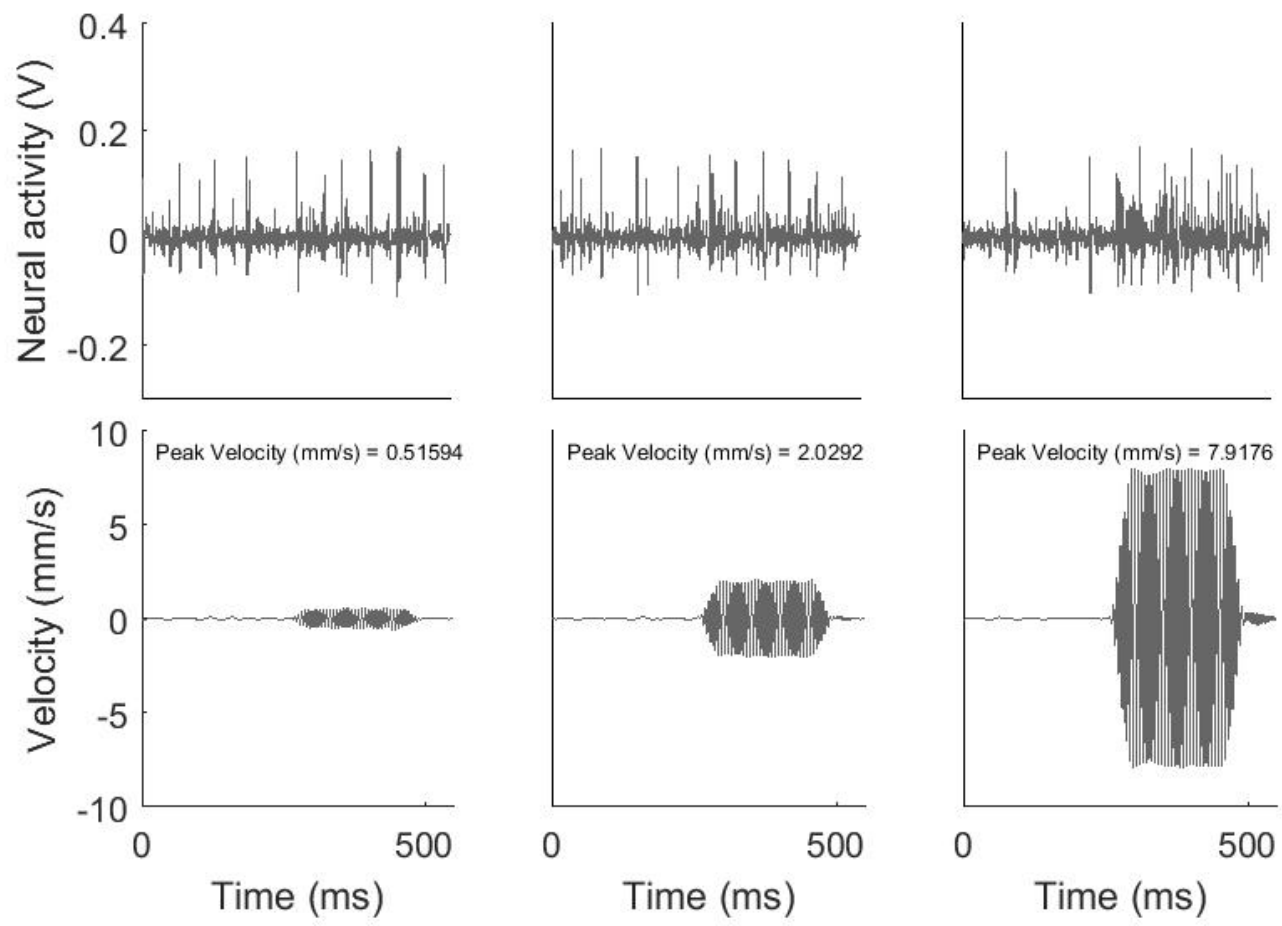

Frequency of $320 \mathrm{~Hz}$ :
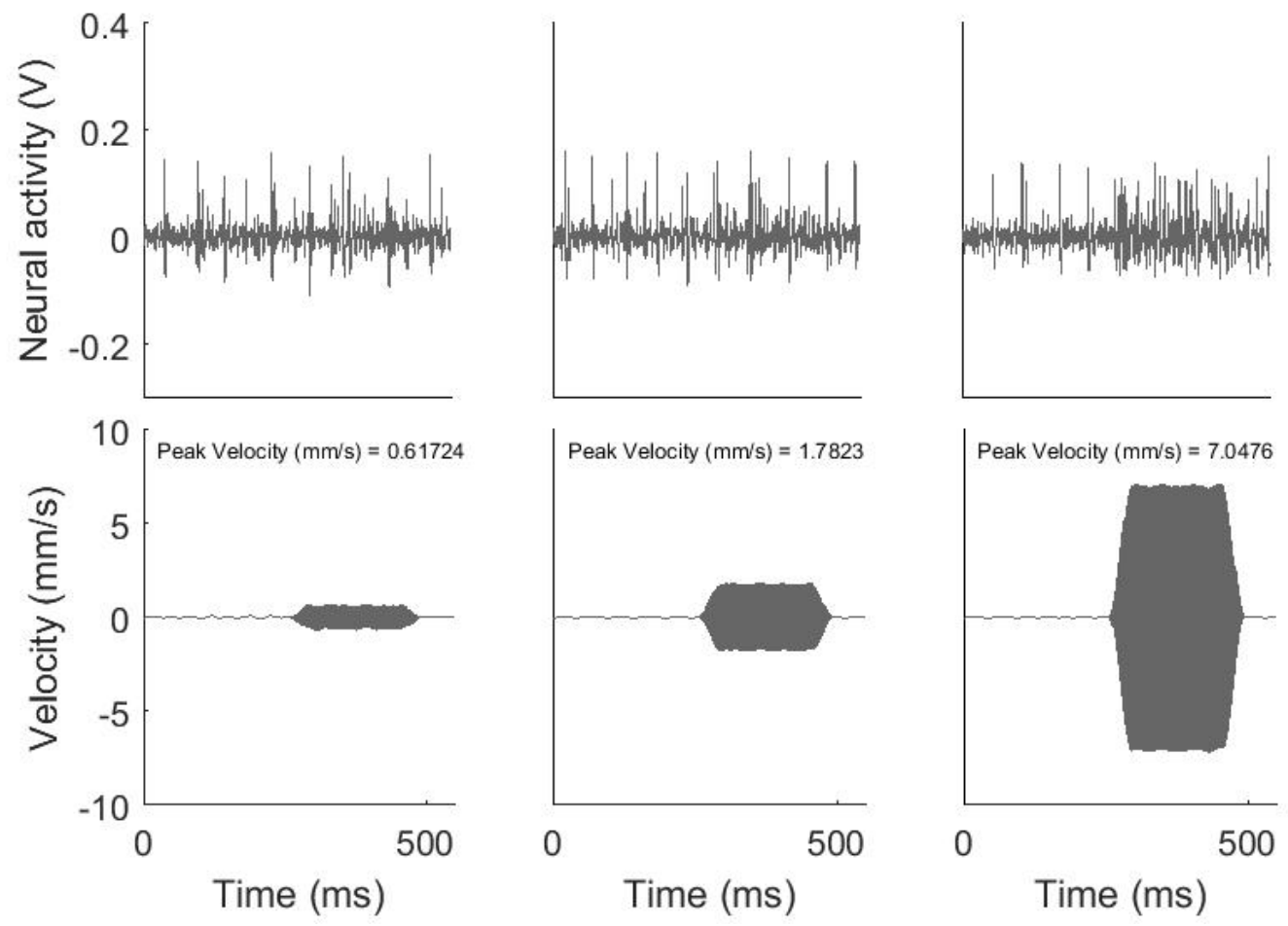


\section{Frequency of $640 \mathrm{~Hz}$ :}
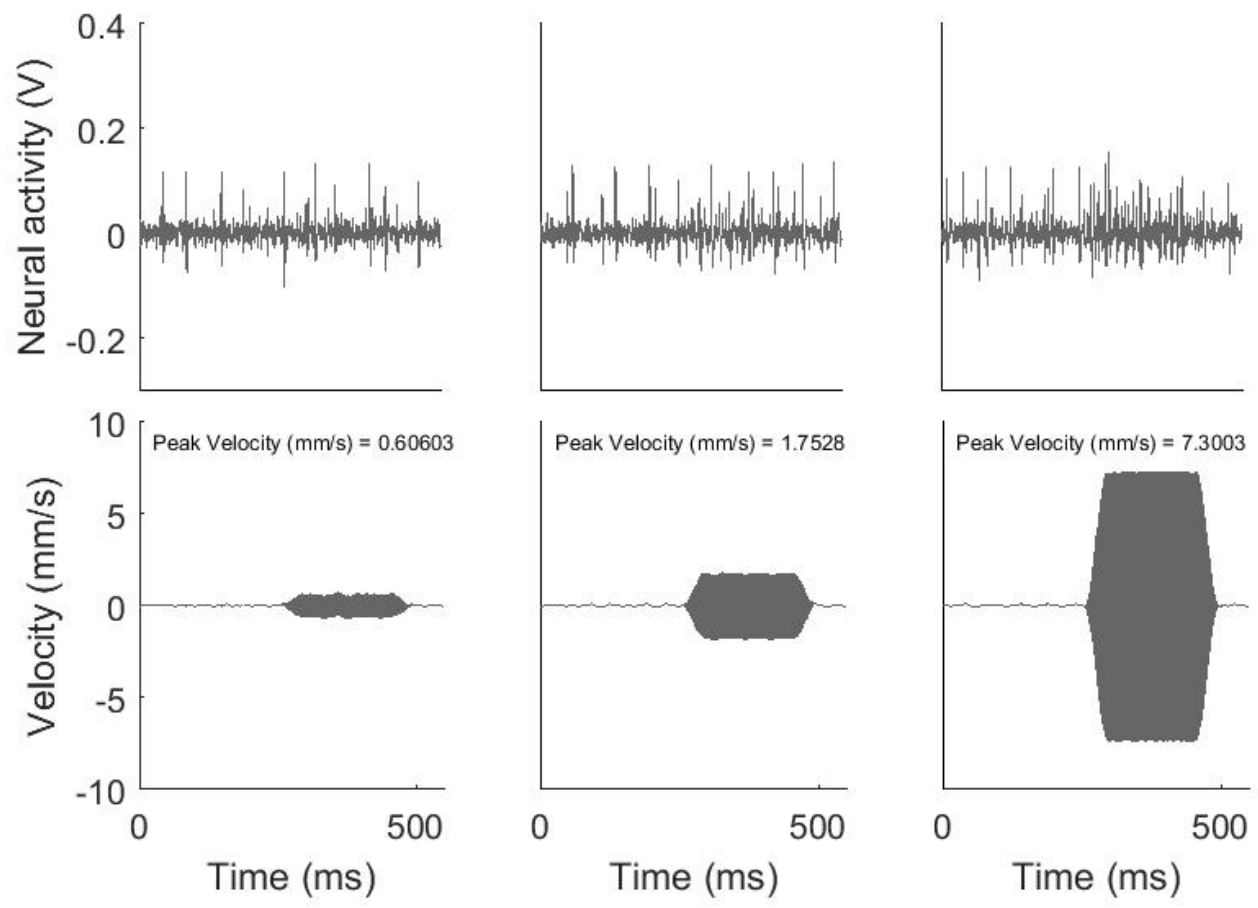

Frequency of $905 \mathrm{~Hz}$ :
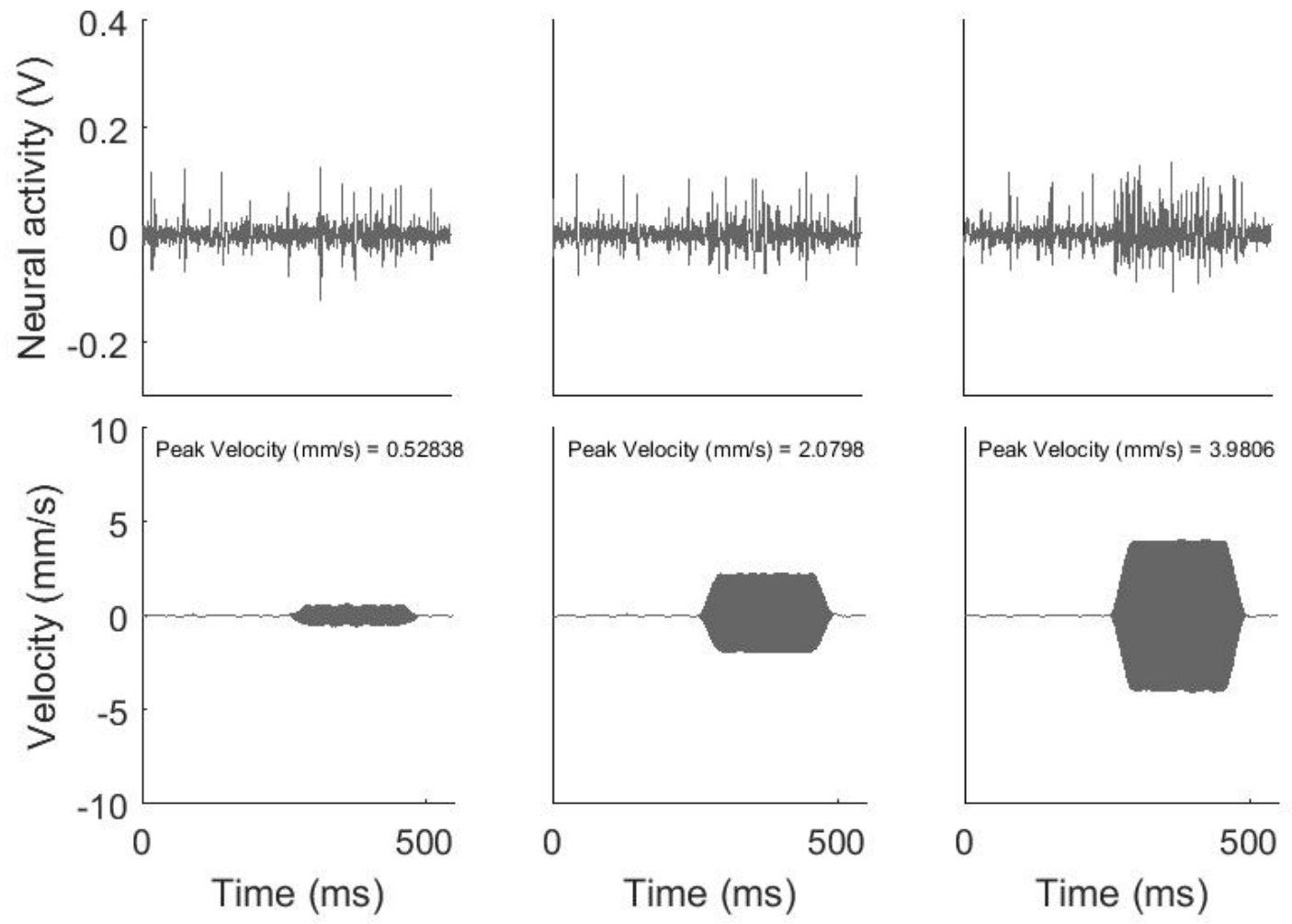
Appendix A.4 - Phase locking for frequencies 20, 80 and $640 \mathrm{~Hz}$ in all T. $n i$ caterpillars

Phase locking for frequencies 20,80 and $640 \mathrm{~Hz}$ for all T. $n i$ caterpillars. Assigned spike angles obtained from neural responses to stimuli of $20 \mathrm{~Hz}$ (top), $80 \mathrm{~Hz}$ (middle) and 640 $\mathrm{Hz}$ (bottom) were plotted in rose histograms. Small rose histograms represent individual caterpillars and large ones summarize the largest bins observed across caterpillars (blue arrow). Red arrows point individual caterpillars that contributed directly to the largest bins, whereas green arrows point caterpillars that did not contribute directly but still followed a similar pattern. 


\section{Frequency of $20 \mathrm{~Hz}$ :}
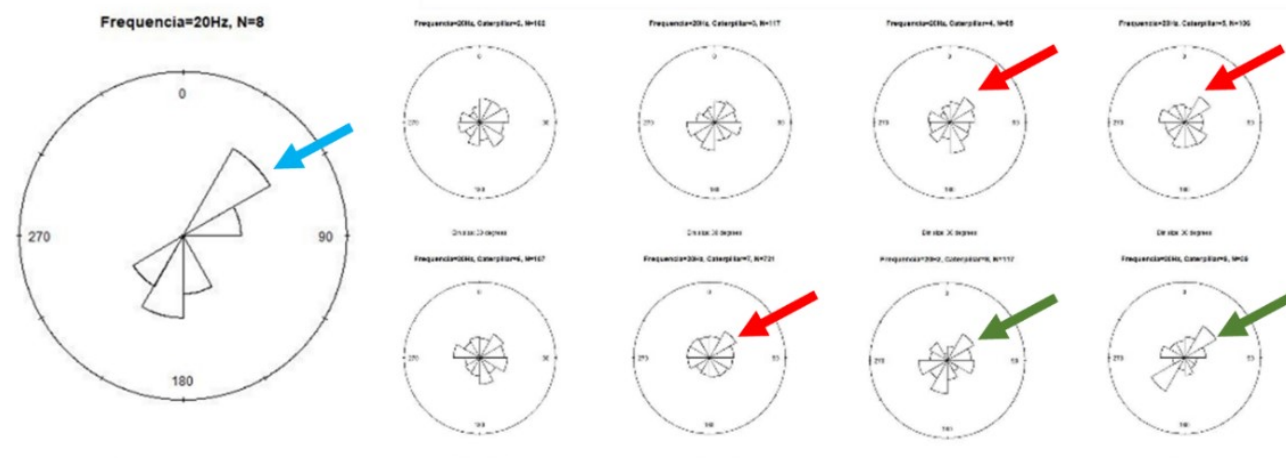

Bin size: 30 segrees

sumsomen

smaxom
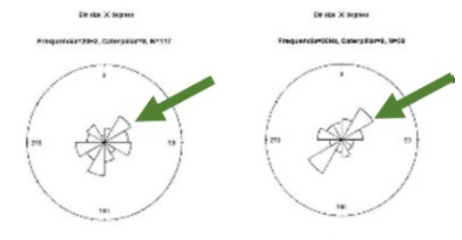

Frequency of $80 \mathrm{~Hz}$ :

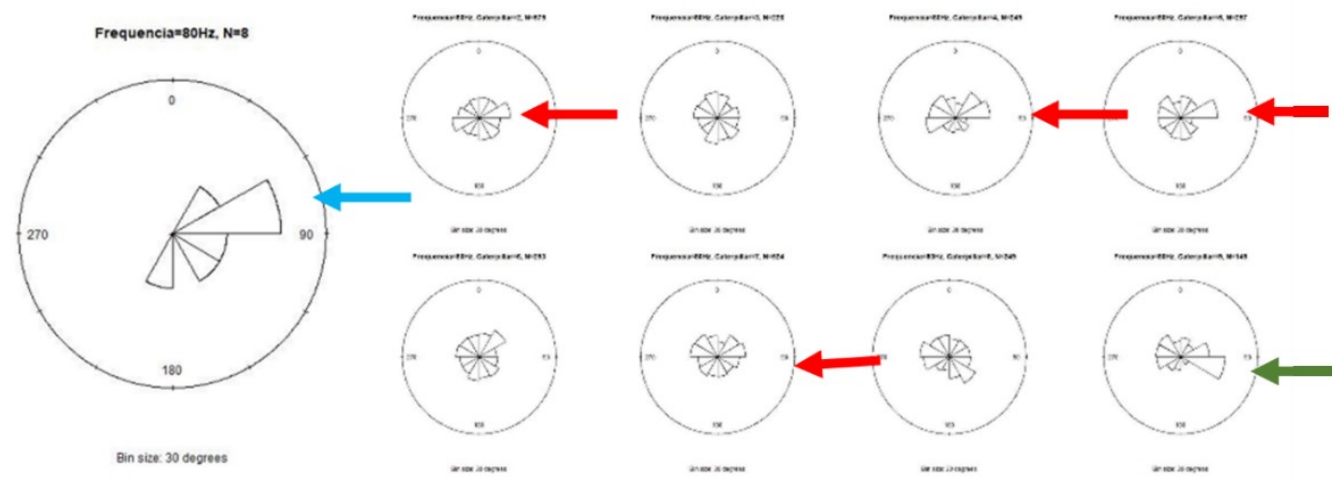

Frequency of $640 \mathrm{~Hz}$ :
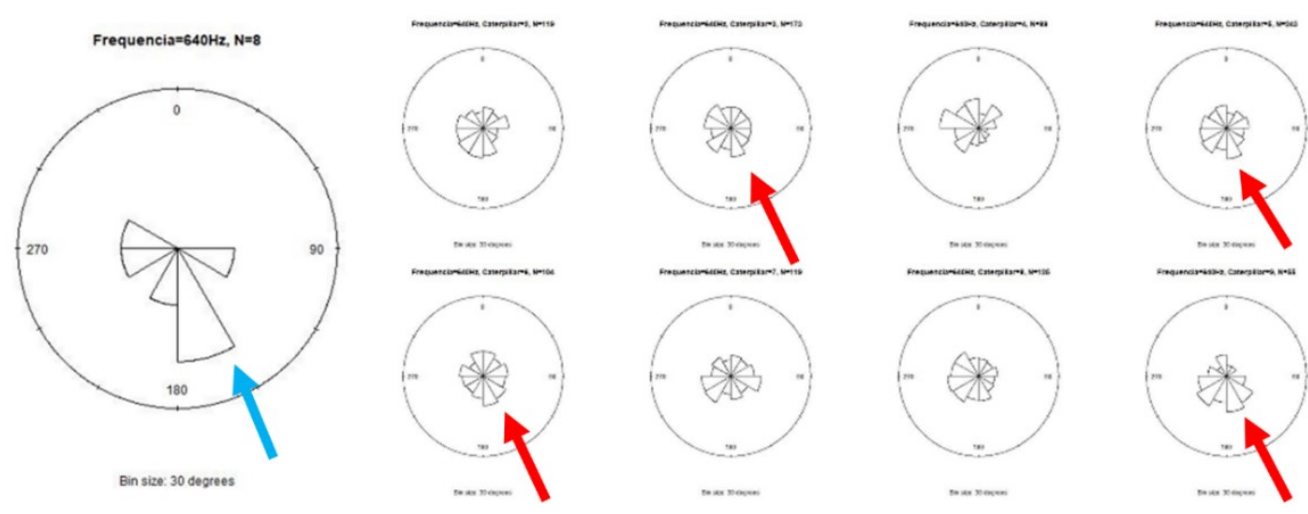
Appendix A.5 - Phase locking for frequencies from 10 to $120 \mathrm{~Hz}$ in one $T$. $n i$ caterpillar

Phase locking for frequencies from 10 to $120 \mathrm{~Hz}$ in $T$. ni. All recordings were made from caterpillar \#7 after its ventral nerve was de-efferented. Each figure shows the vibrational signal that was applied repeatedly to the hair (bottom window), an individual trace of the response (middle window), all responses overlaid (grey lines that look like a continuous grey area on top window) and an average of all responses (dark line on top window). Dashed lines indicate the moments at which the stimulus reaches peak velocities, which correspond to a phase angle of $0 / 360^{\circ}$. The rose histogram below each graph summarizes the phases at which the spikes happened. The number of signals used in the analysis for each frequency is indicated on the top window of the graphs, while the number of spikes counted is indicated on the title of the rose histograms. 
Frequency of $10 \mathrm{~Hz}$ :

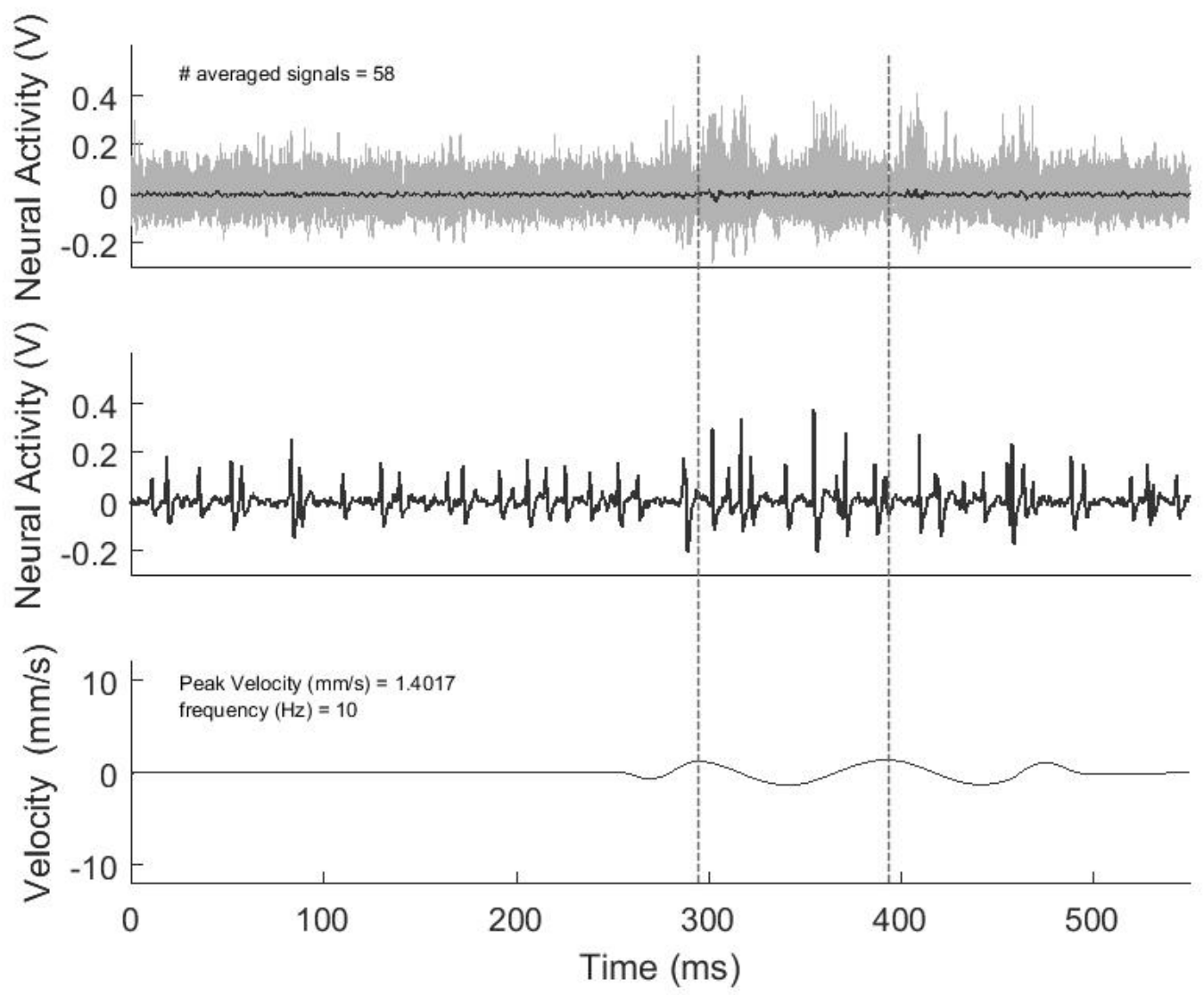

Frequency $=10 \mathrm{~Hz}$, Caterpillar $=7, \mathrm{~N}=402$

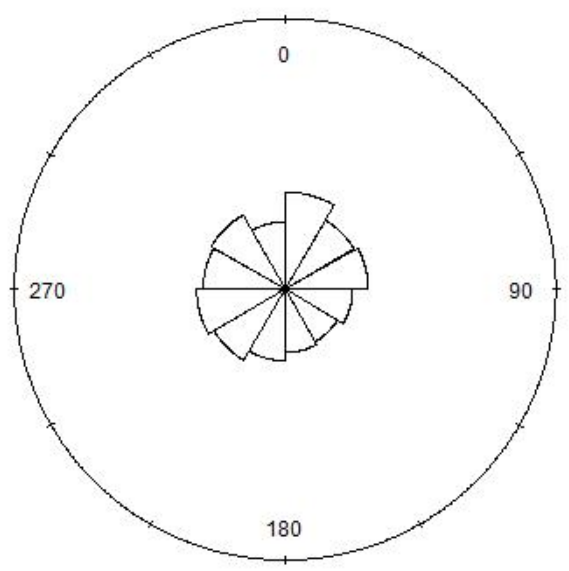

Bin size: 30 degrees 
Frequency of $20 \mathrm{~Hz}$ :

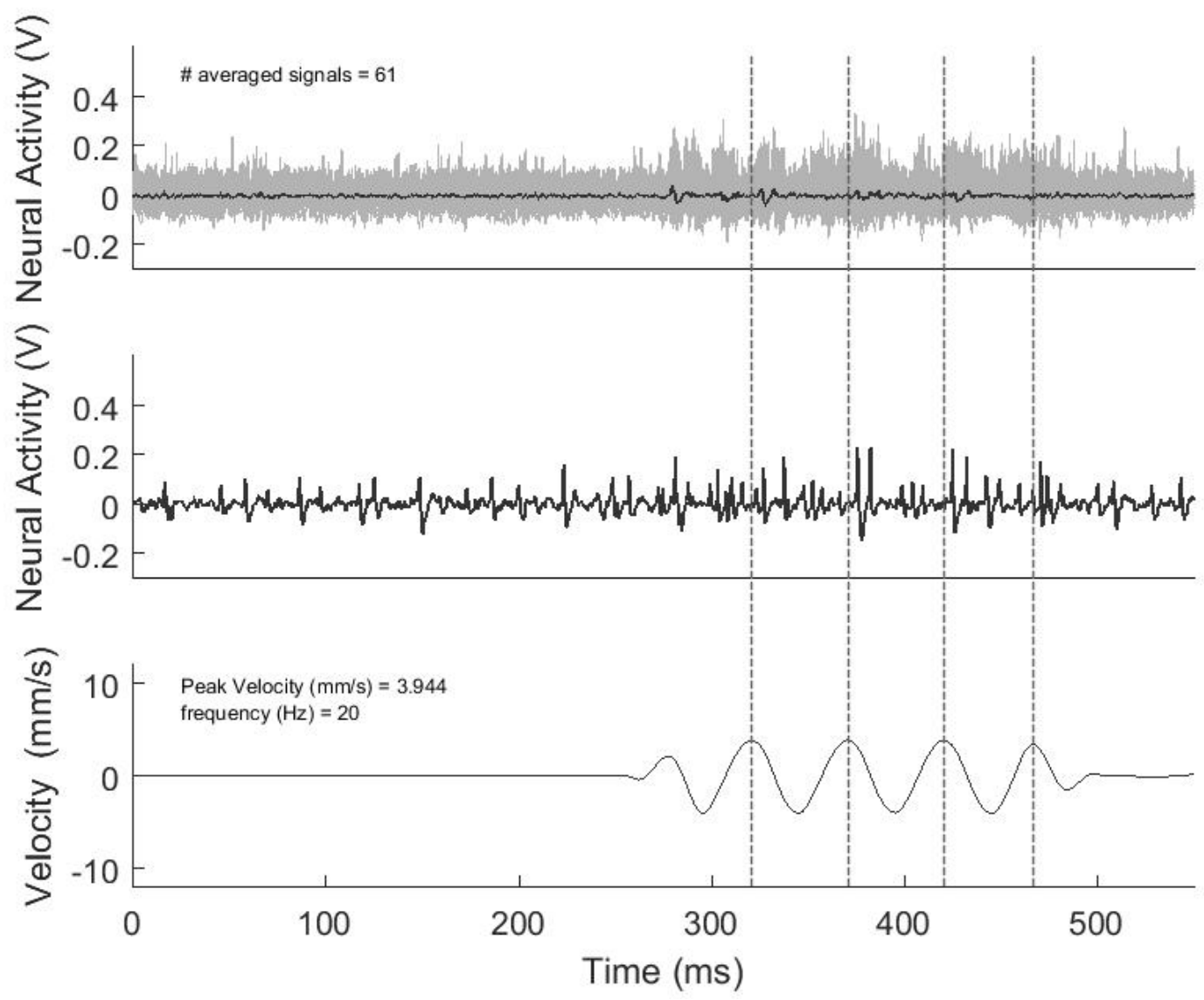

Frequency $=\mathbf{2 0 H z}$, Caterpillar $=7, \mathrm{~N}=\mathbf{6 2 3}$

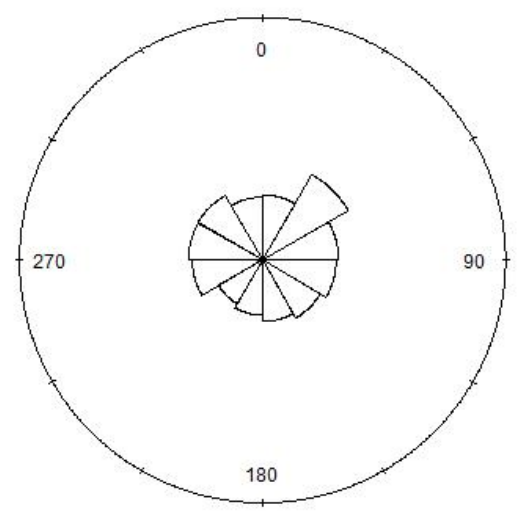

Bin size: 30 degrees 


\section{Frequency of $50 \mathrm{~Hz}$ :}

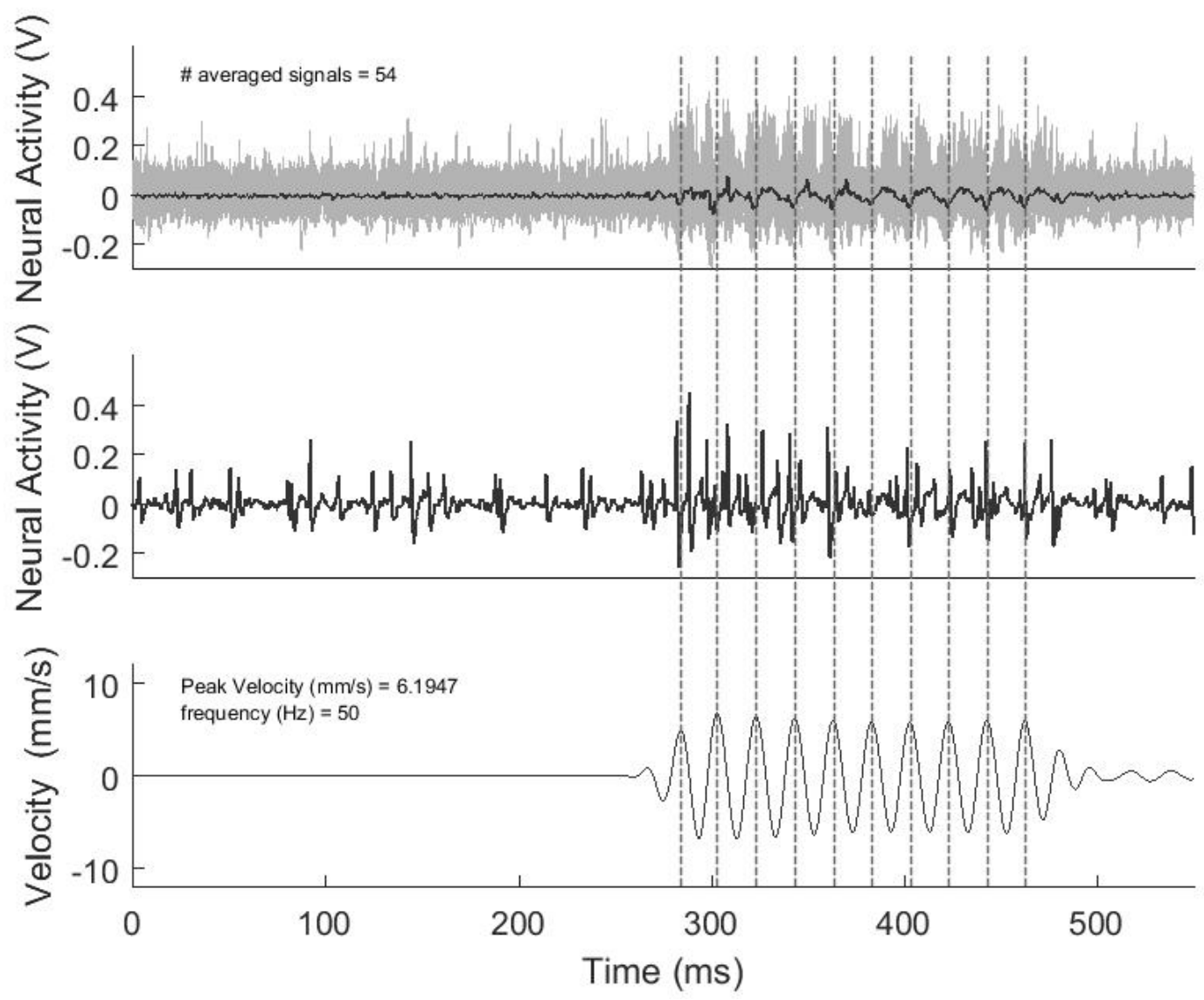

Frequency $=50 \mathrm{~Hz}$, Caterpillar $=7, \mathrm{~N}=1275$

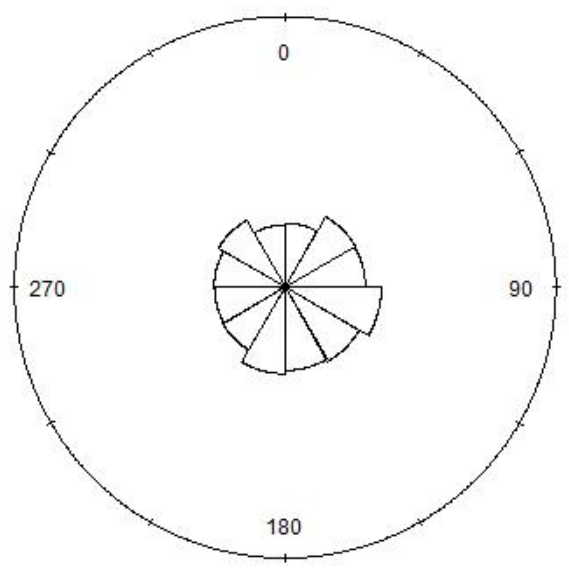

Bin size: 30 degrees 


\section{Frequency of $60 \mathrm{~Hz}$ :}

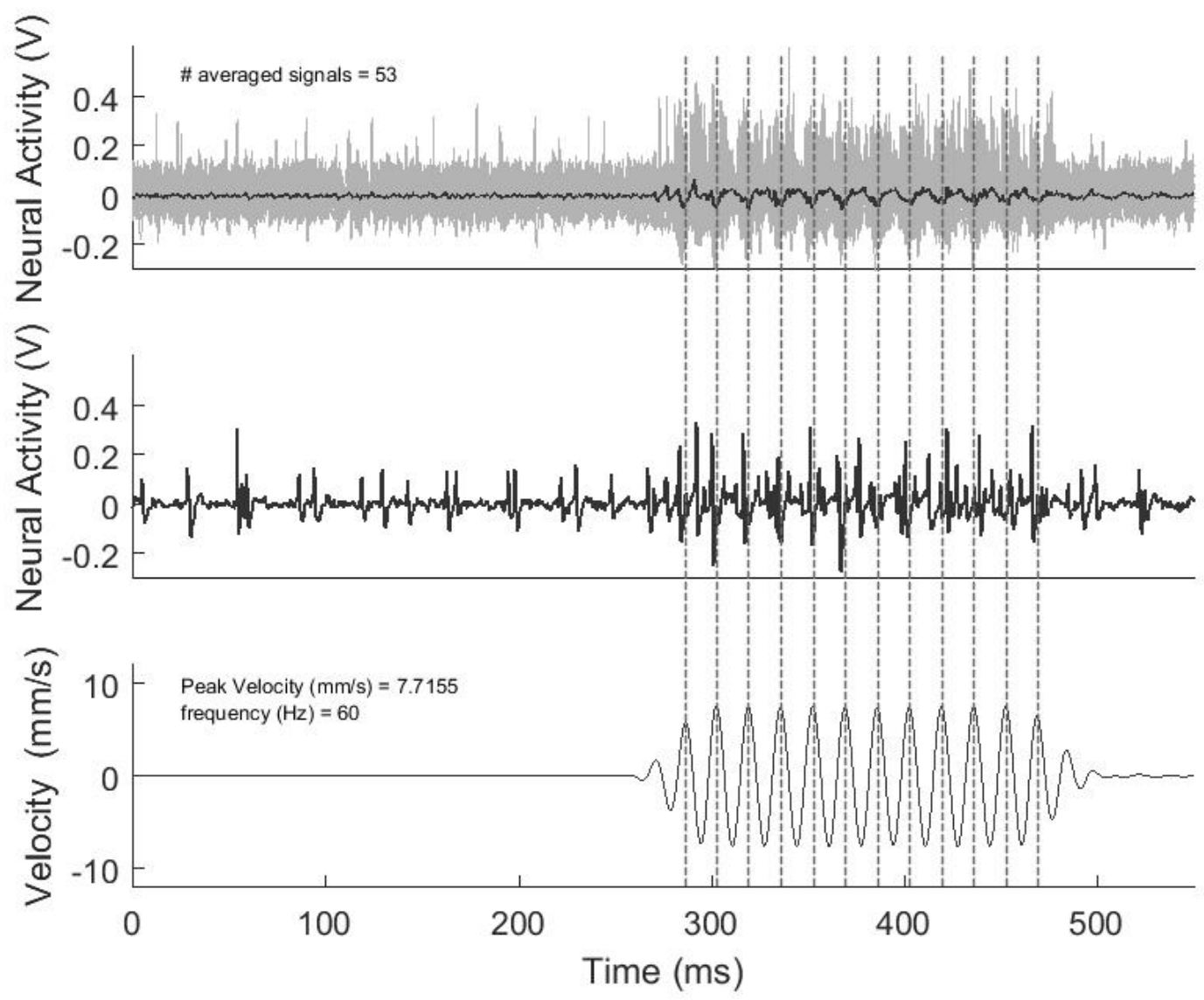

Frequency $=60 \mathrm{~Hz}$, Caterpillar $=7, \mathrm{~N}=1544$

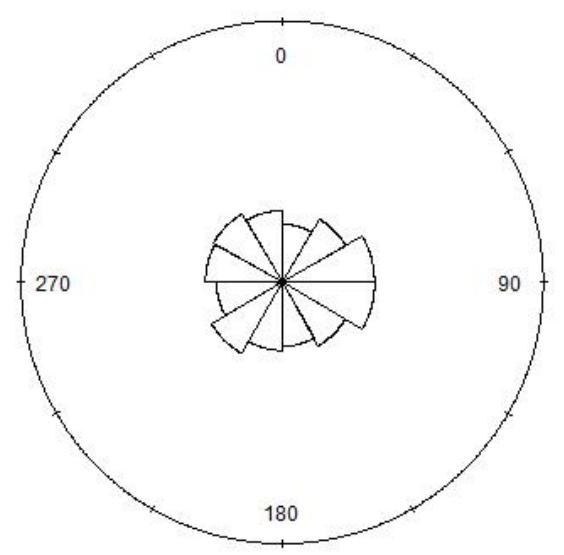

Bin size: 30 degrees 


\section{Frequency of $70 \mathrm{~Hz}$ :}

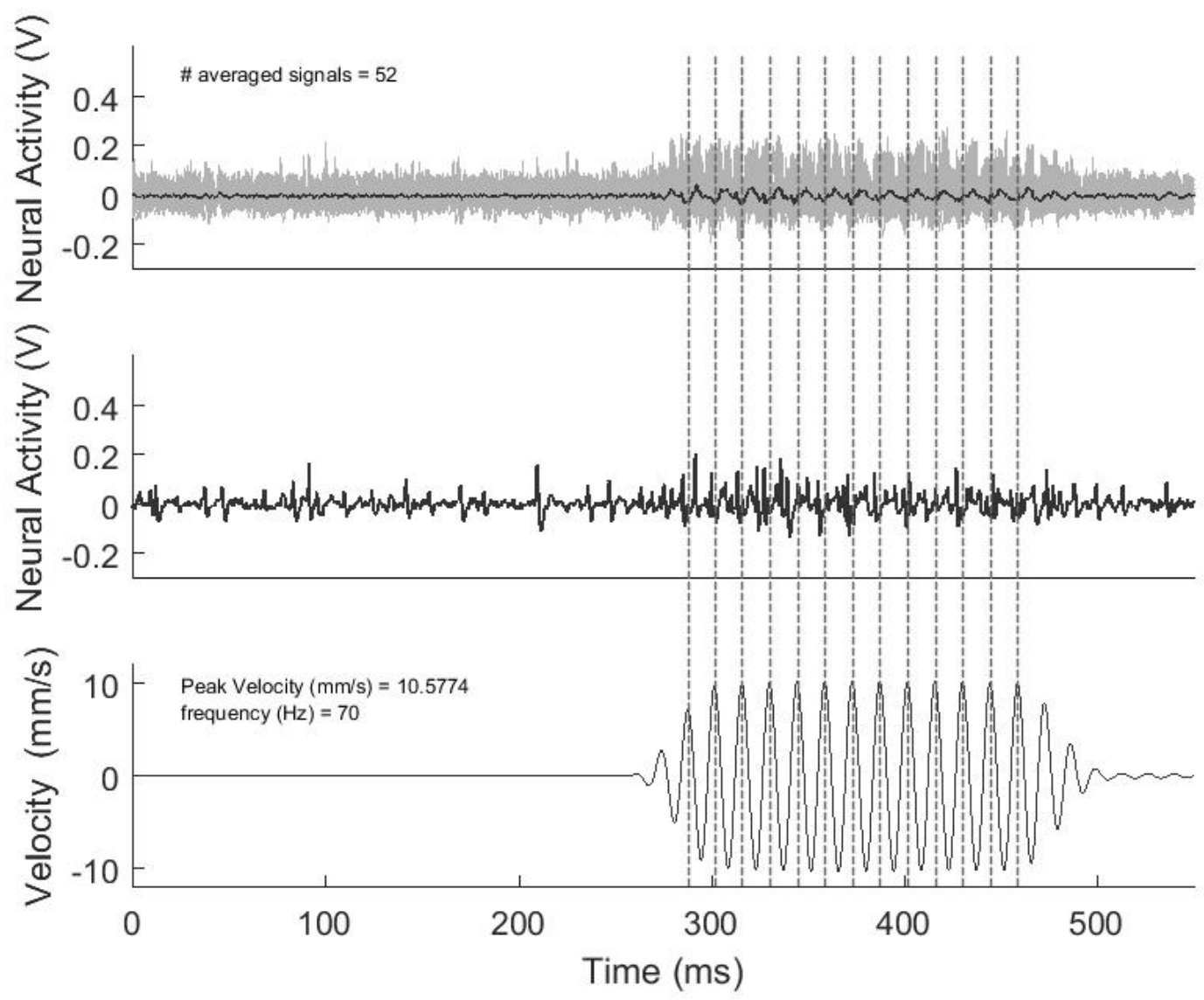

Frequency $=70 \mathrm{~Hz}$, Caterpillar=7, N=1414

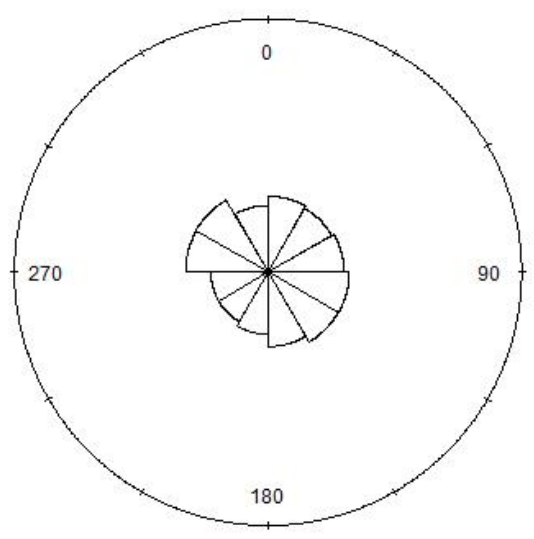

Bin size: 30 degrees 


\section{Frequency of $80 \mathrm{~Hz}$ :}

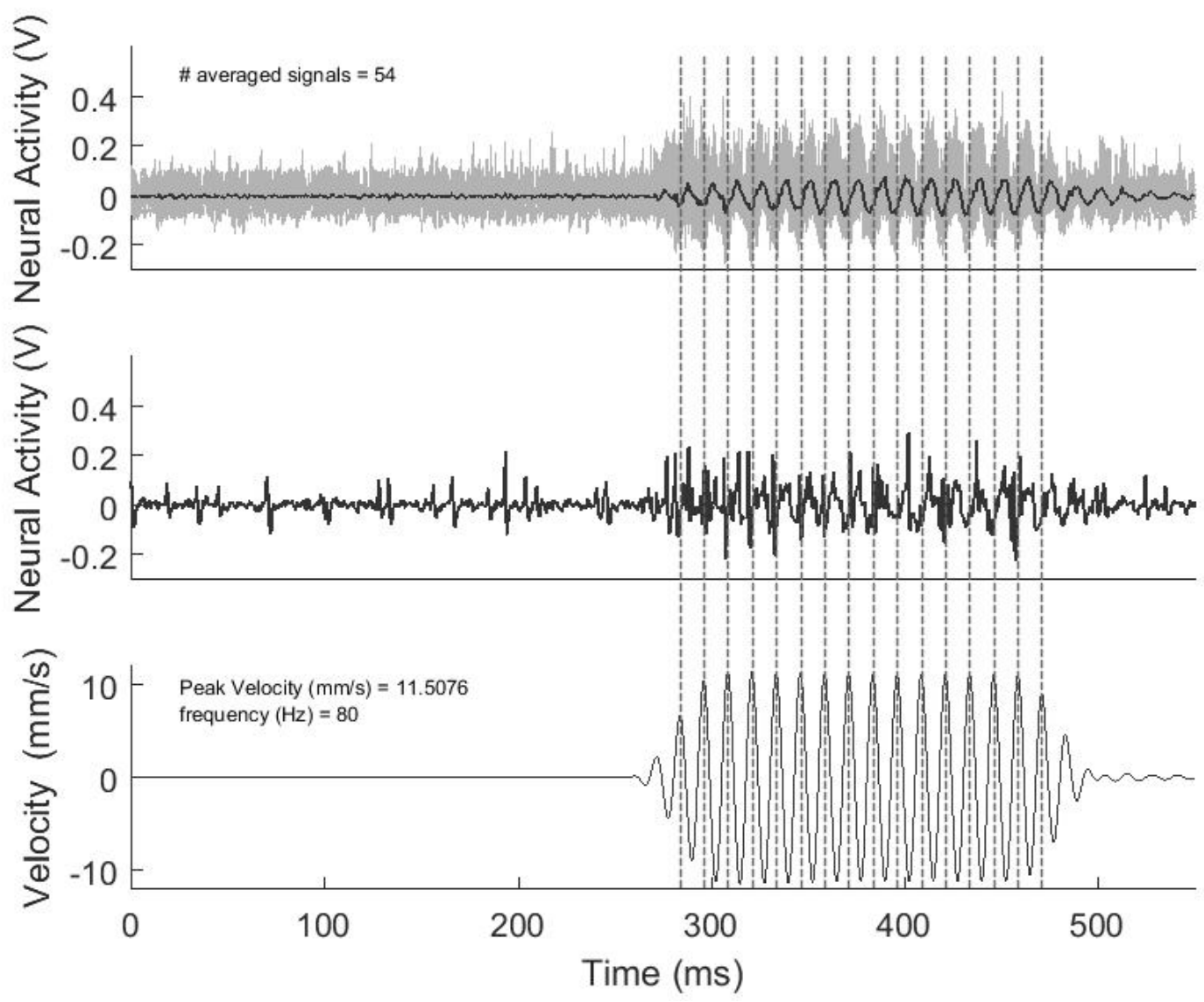

Frequency $=80 \mathrm{~Hz}$, Caterpillar $=7, \mathrm{~N}=1430$

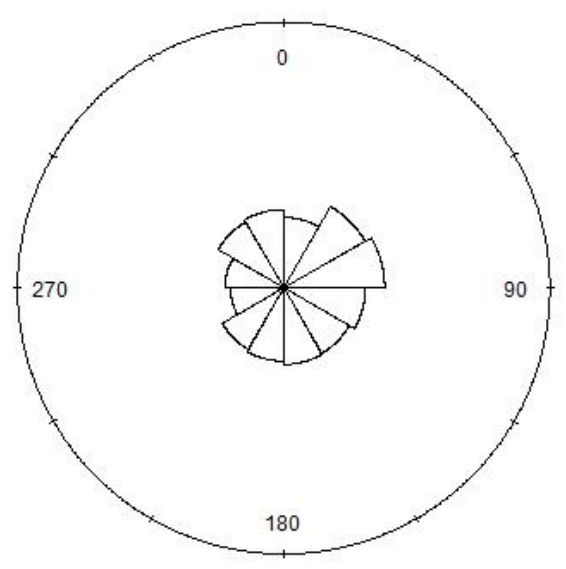

Bin size: 30 degrees 


\section{Frequency of $90 \mathrm{~Hz}$ :}

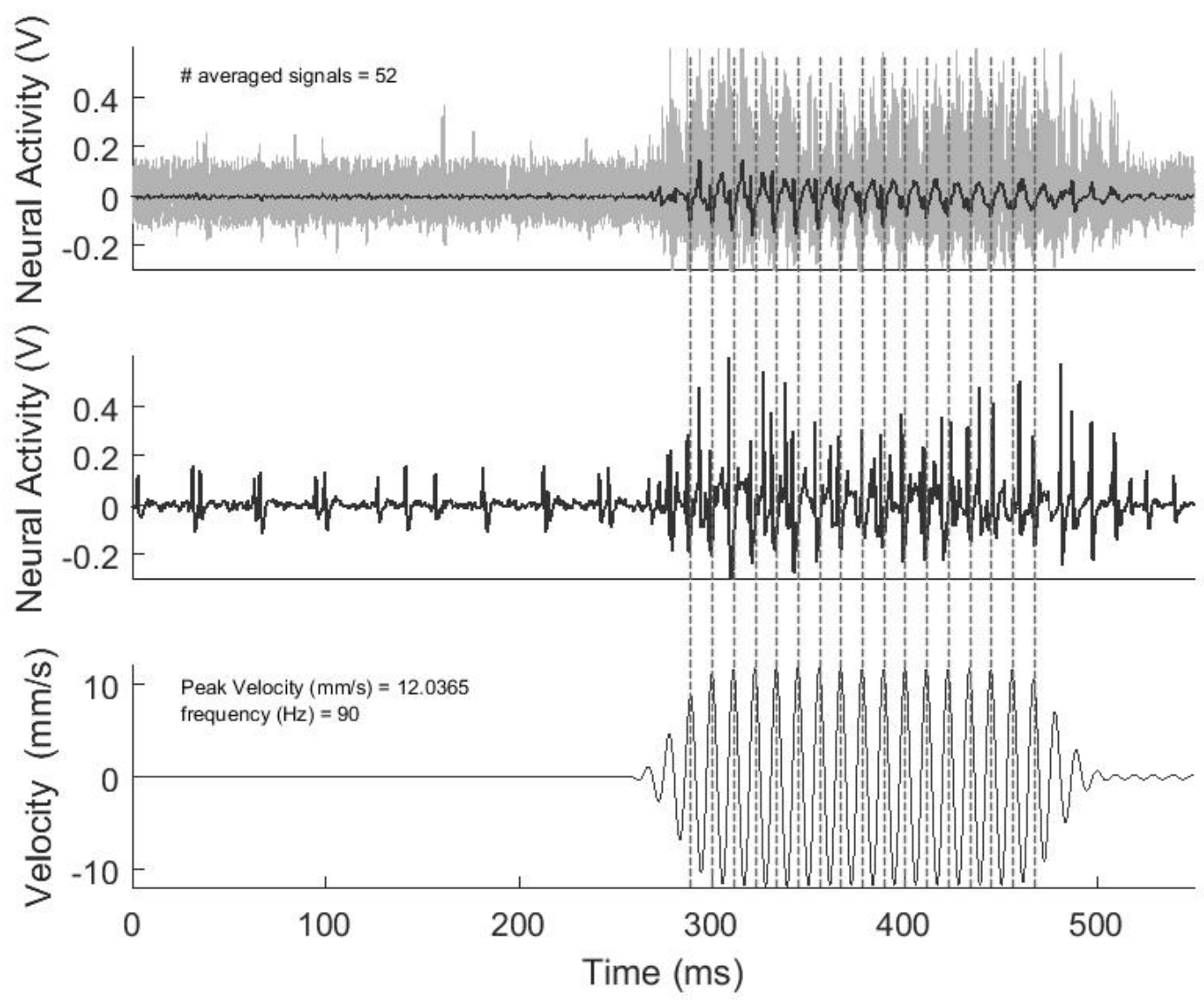

Frequency $=90 \mathrm{~Hz}$, Caterpillar $=7, \mathrm{~N}=1832$

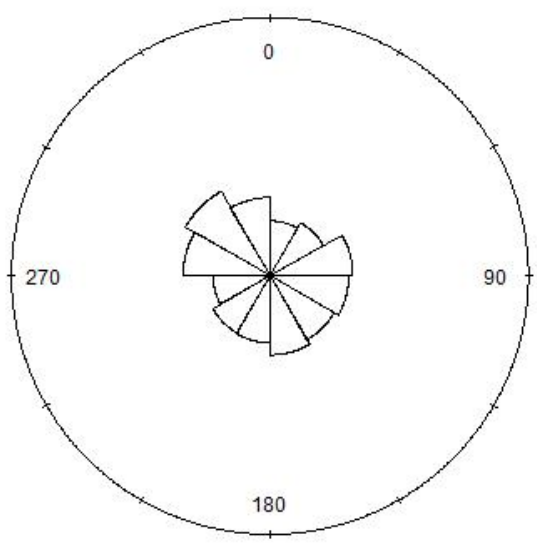

Bin size: 30 degrees 


\section{Frequency of $100 \mathrm{~Hz}$ :}

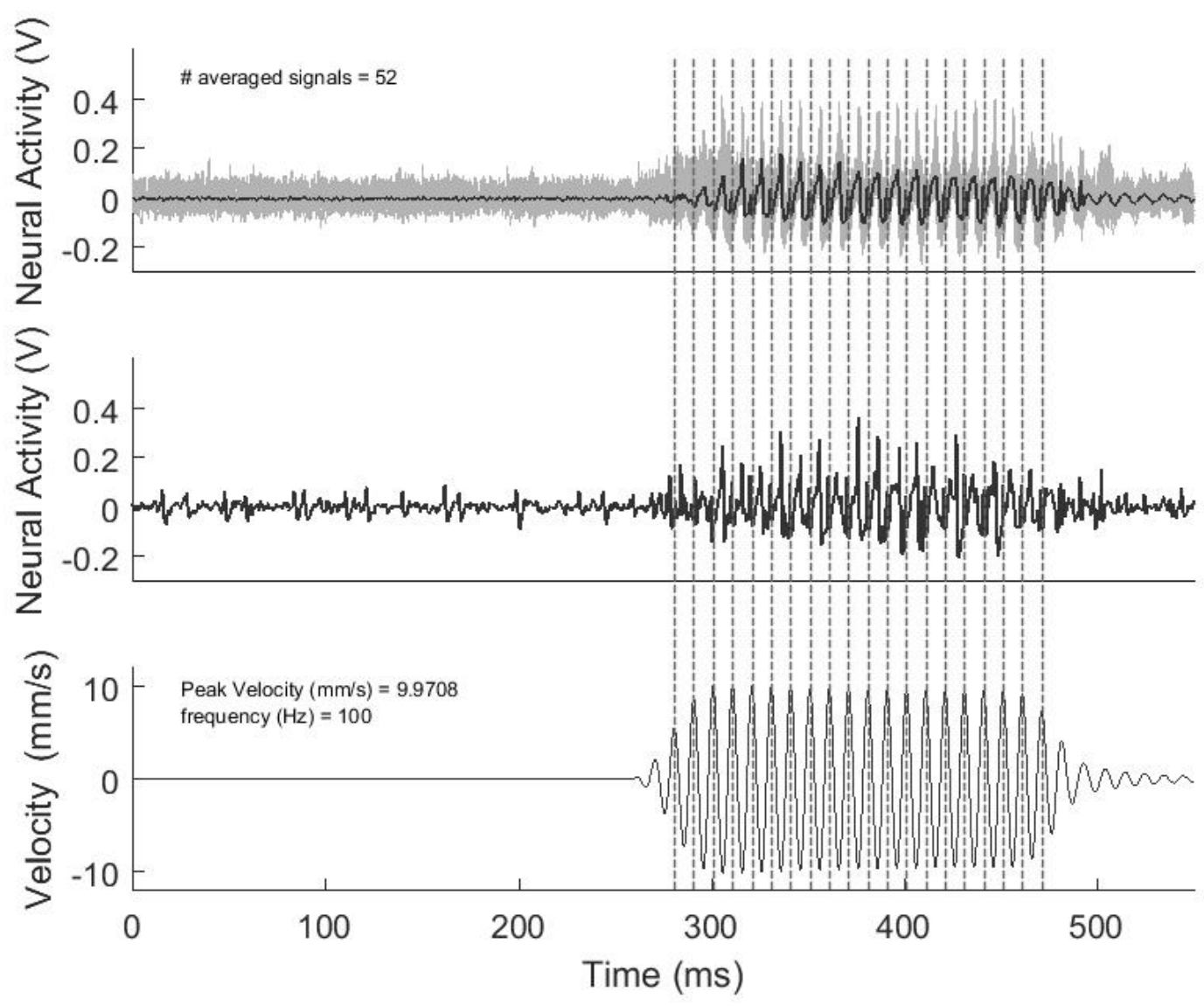

Frequency $=100 \mathrm{~Hz}$, Caterpillar $=7, \mathrm{~N}=\mathbf{2 0 1 0}$

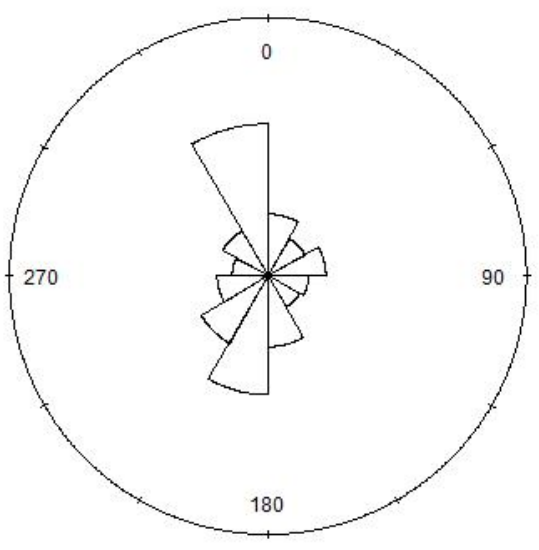

Bin size: 30 degrees 


\section{Frequency of $110 \mathrm{~Hz}$ :}

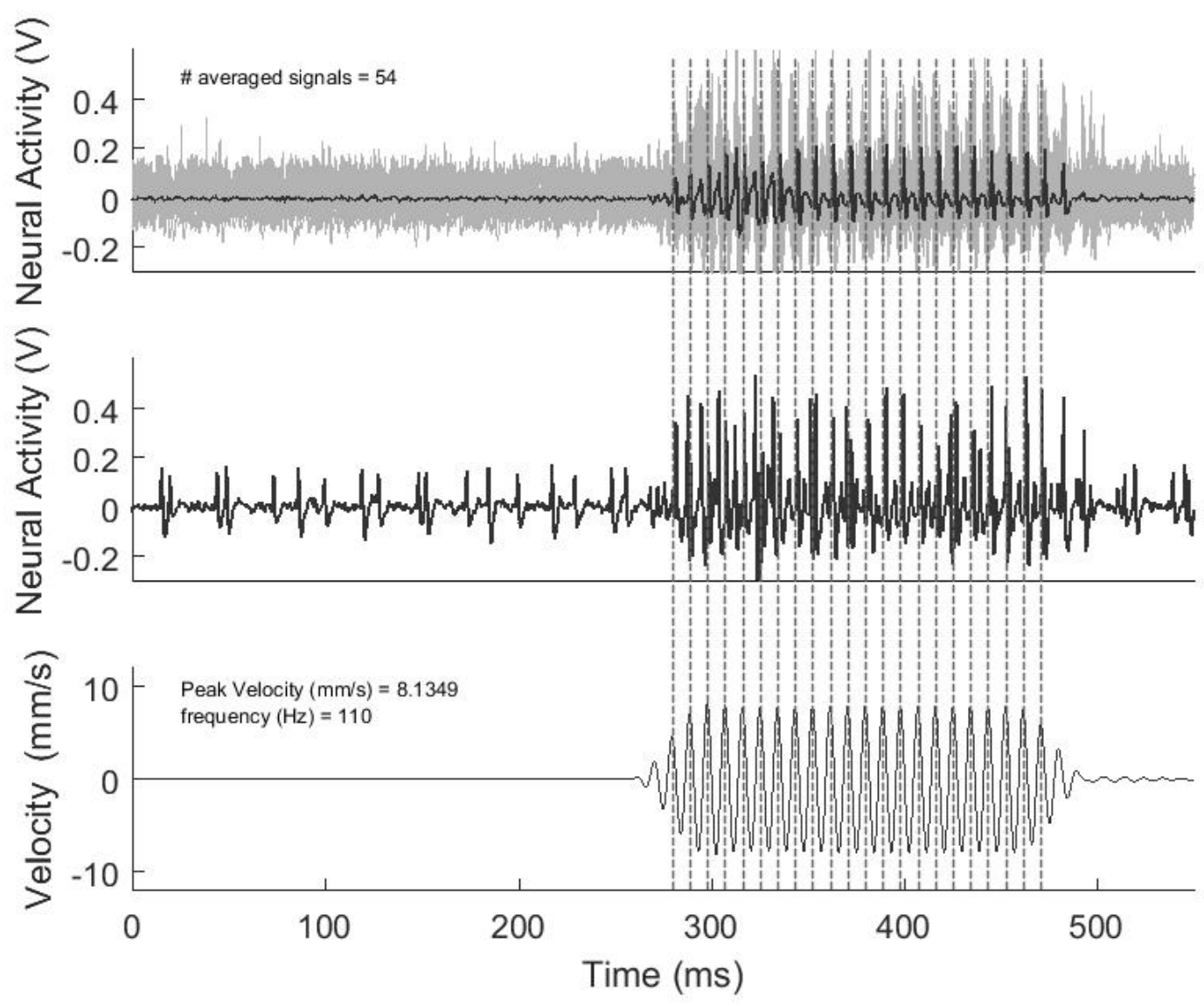

Frequency $=110 \mathrm{~Hz}$, Caterpillar $=7, \mathrm{~N}=2402$

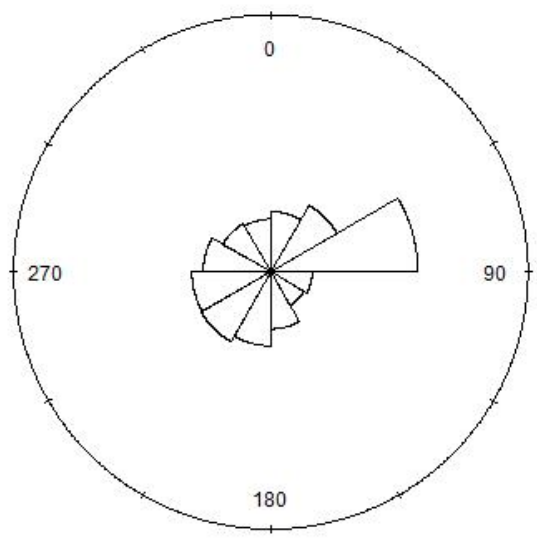

Bin size: 30 degrees 


\section{Frequency of $120 \mathrm{~Hz}$ :}

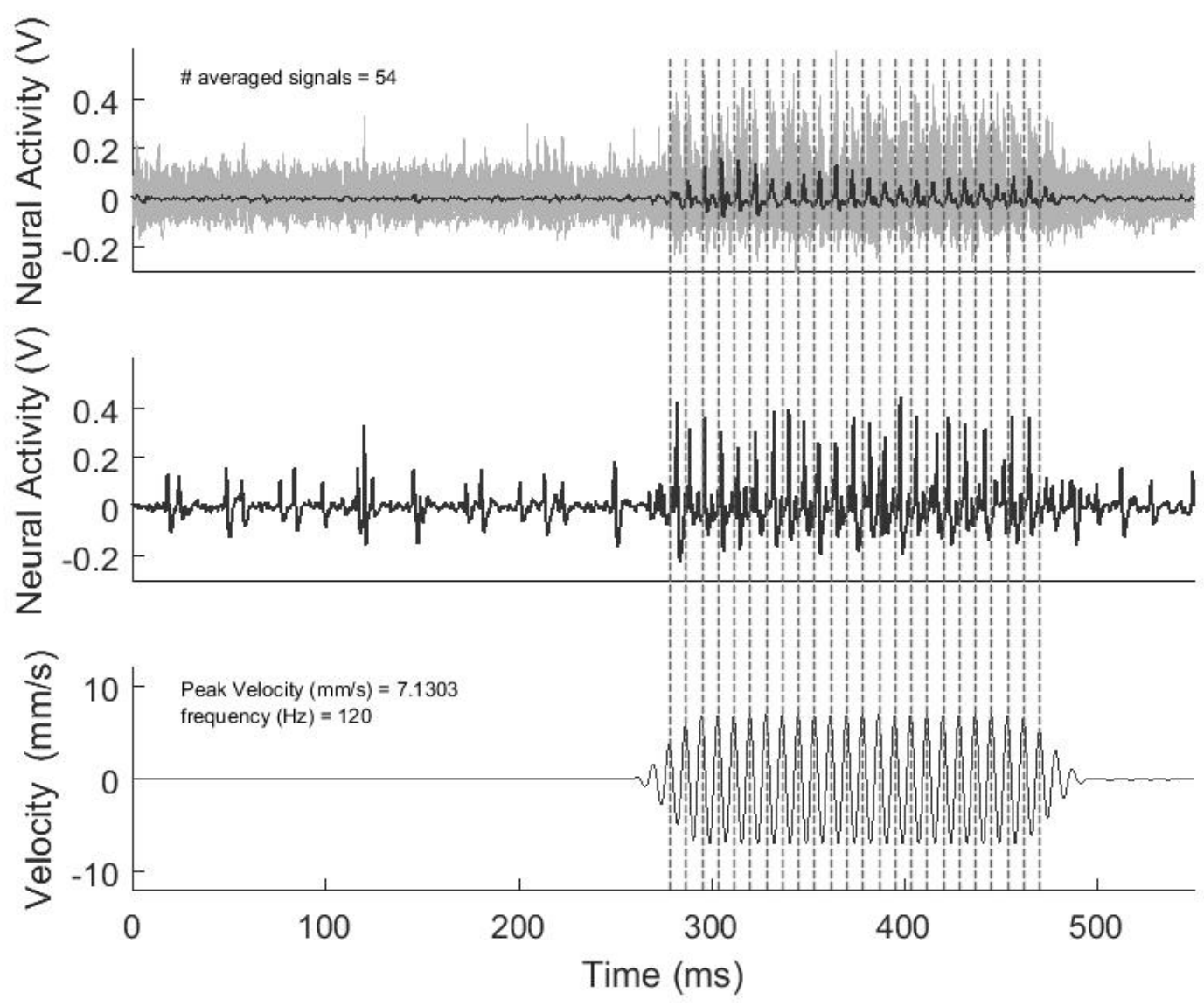

Frequency $=\mathbf{1 2 0 \mathrm { Hz }}$, Caterpillar $=\mathbf{7}, \mathrm{N}=\mathbf{2 0 0 0}$

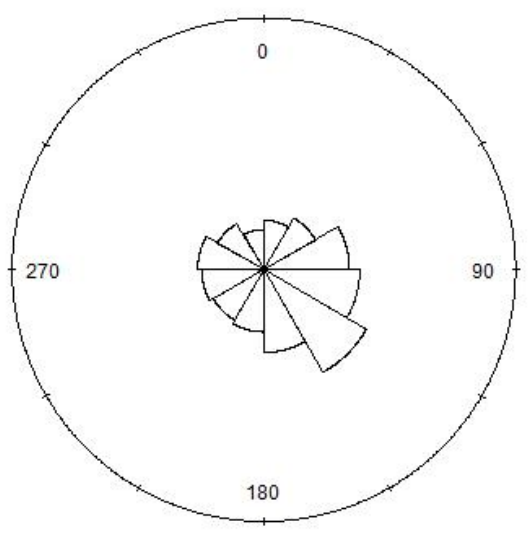

Bin size: 30 degrees 


\section{Appendix B (Supplements to Chapter 3)}

Appendix B.1 - Local regressions loess() for all frequencies in two D. arcuata caterpillars

Local regressions loess() in two D. arcuata caterpillars for all frequencies. Subplot A refers to $10 \mathrm{~Hz}$ signals applied to the hair of Caterpillar D. Subplots B to D refer to 10 to $640 \mathrm{~Hz}$ signals applied to the waxed setae and cuticle of caterpillar A. Units on the horizontal axes are Peak velocity $(\mathrm{mm} / \mathrm{s})$ and on the vertical axes are difference in \# spikes during minus before stimuli. Each data point represents one stimulus signal. Black, dark grey and light grey lines represent the fit, 99\% confidence intervals and $95 \%$ predict intervals of the local regression, respectively. The dashed line at each subplot indicates the point at which the lower confidence interval surpassed the zero line for the first time, which was defined as the threshold for that specific caterpillar to that frequency. 


\section{Frequencies 10 to $640 \mathrm{~Hz}$}
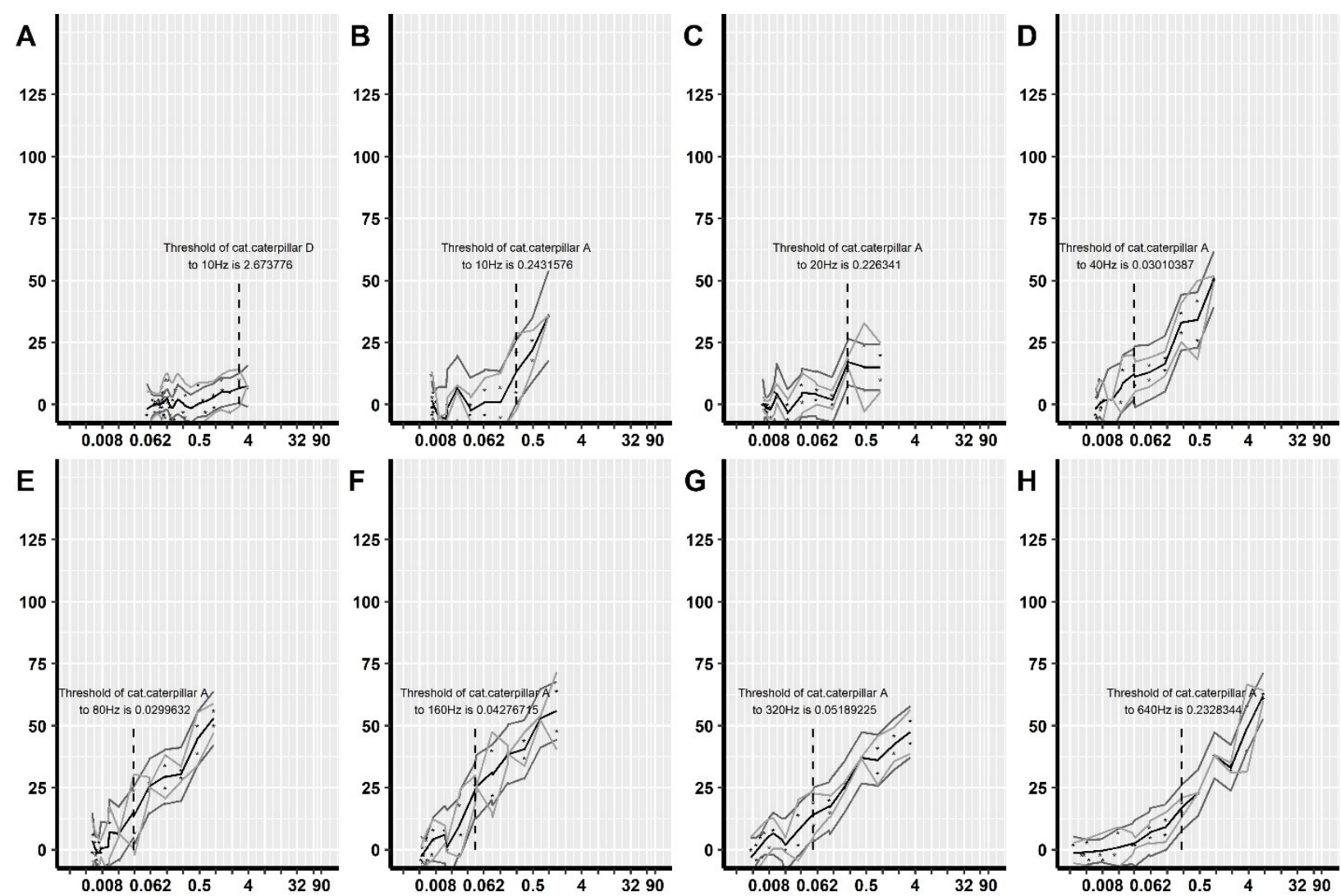
Appendix B.2 - Sample traces of neural activity from waxed cuticle in D. arcuata

Sample traces of neural activity from waxed cuticle and proleg hairs in D. arcuata responding to sine stimuli of different frequencies and intensities. 


\section{Frequency: $10 \mathrm{~Hz}$}
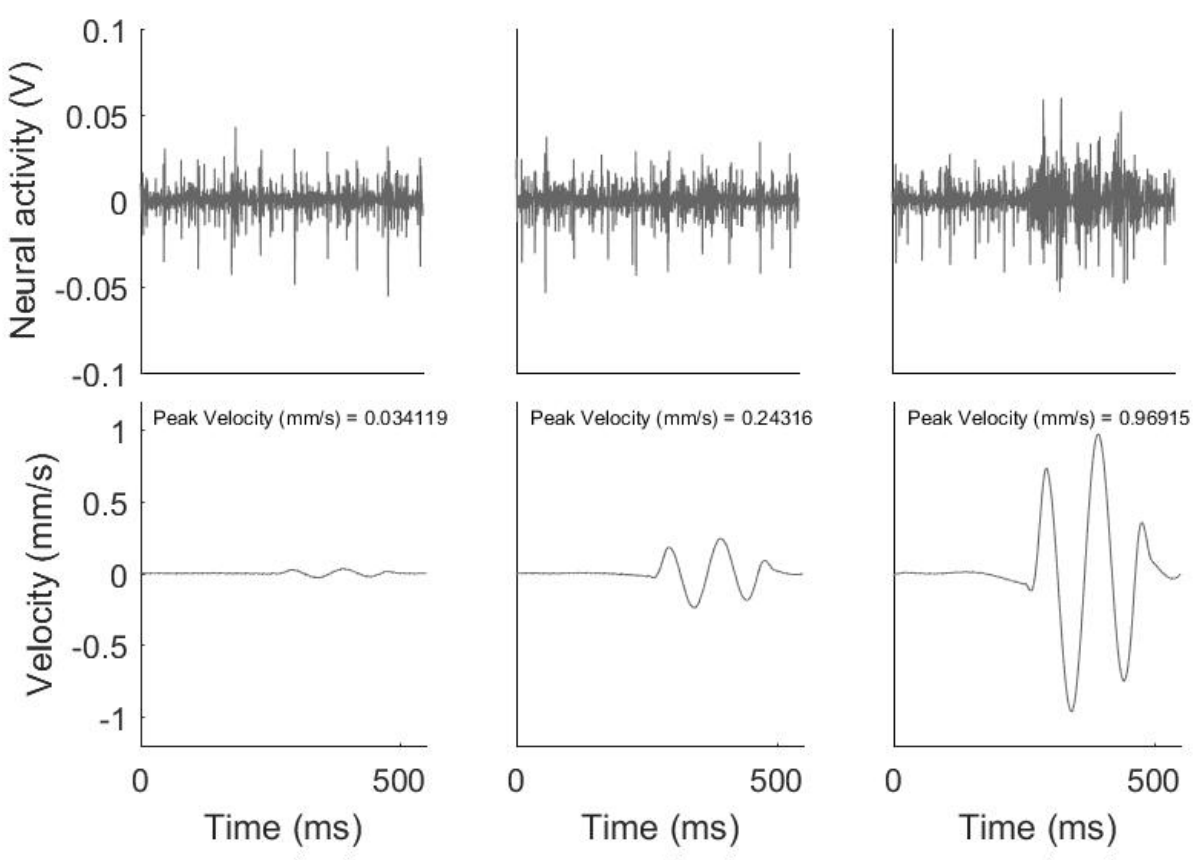

\section{Frequency: $20 \mathrm{~Hz}$}
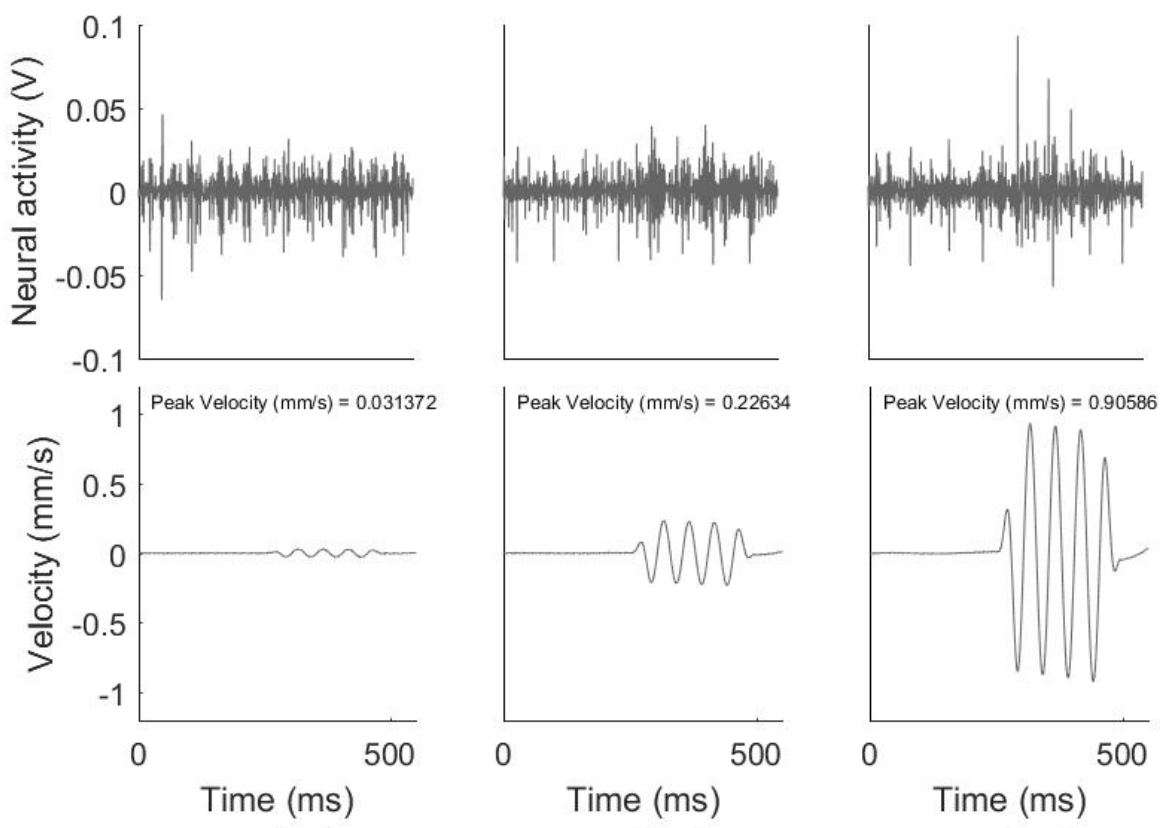


\section{Frequency: $40 \mathrm{~Hz}$}
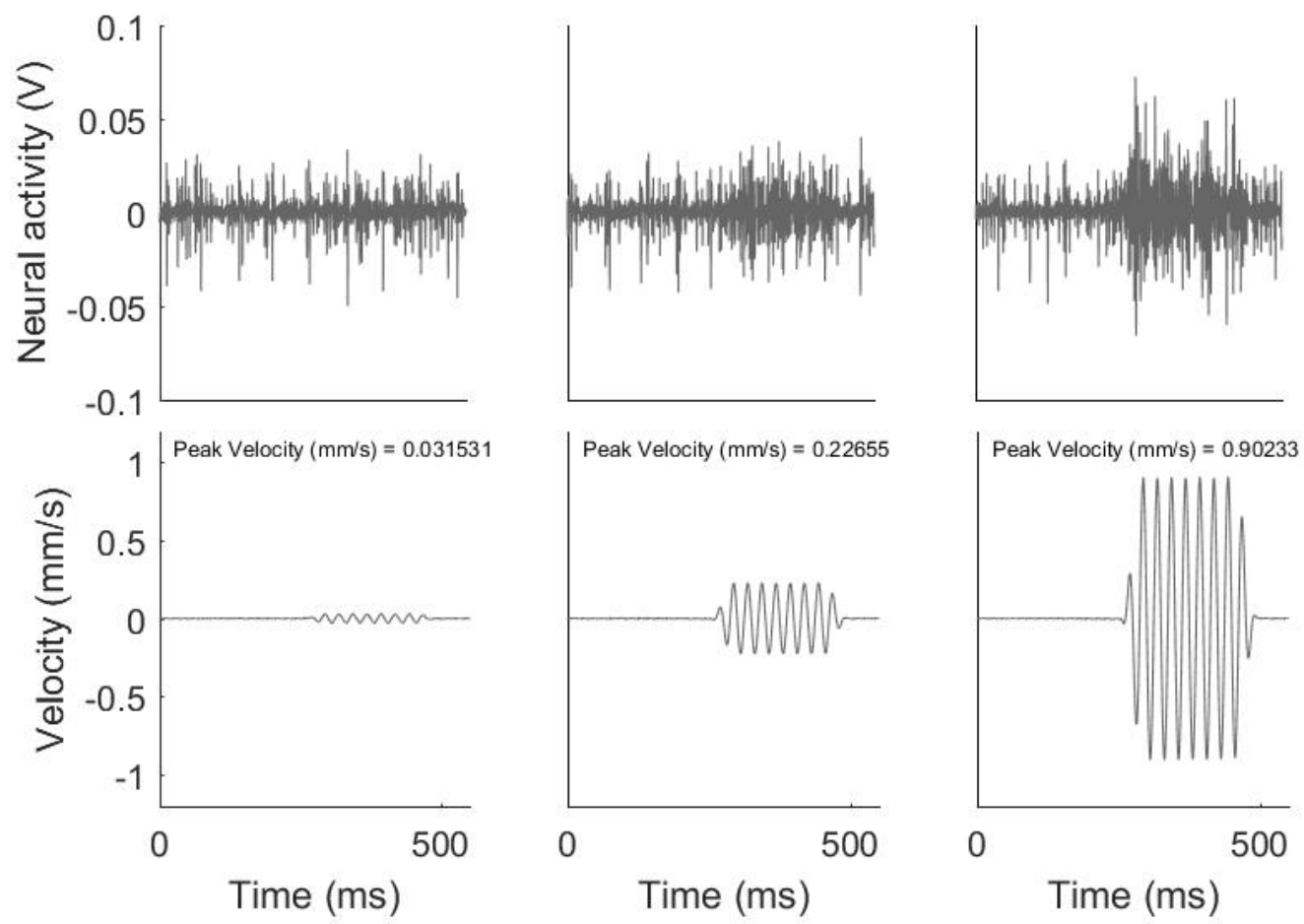

\section{Frequency: $80 \mathrm{~Hz}$}
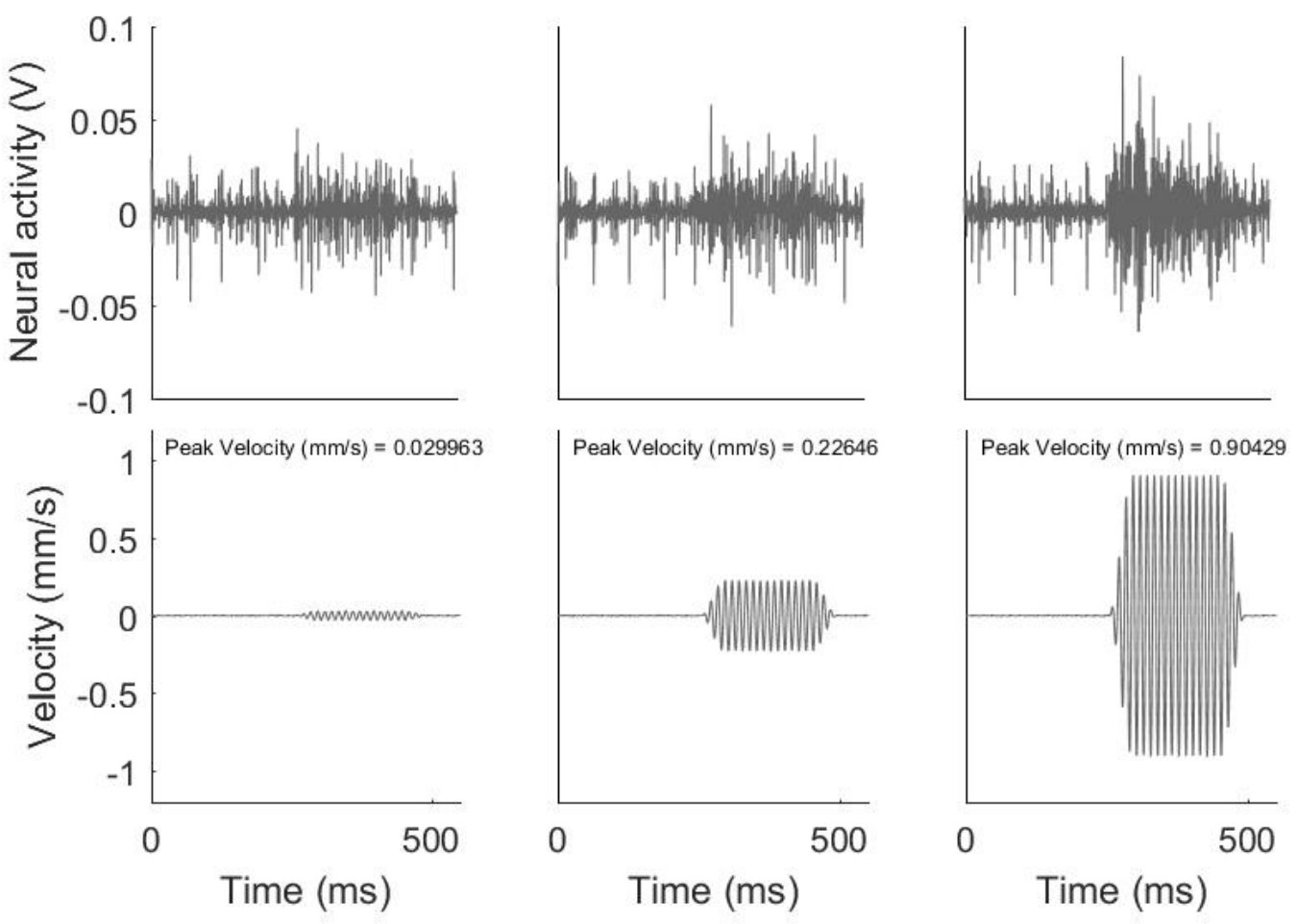


\section{Frequency: $160 \mathrm{~Hz}$}
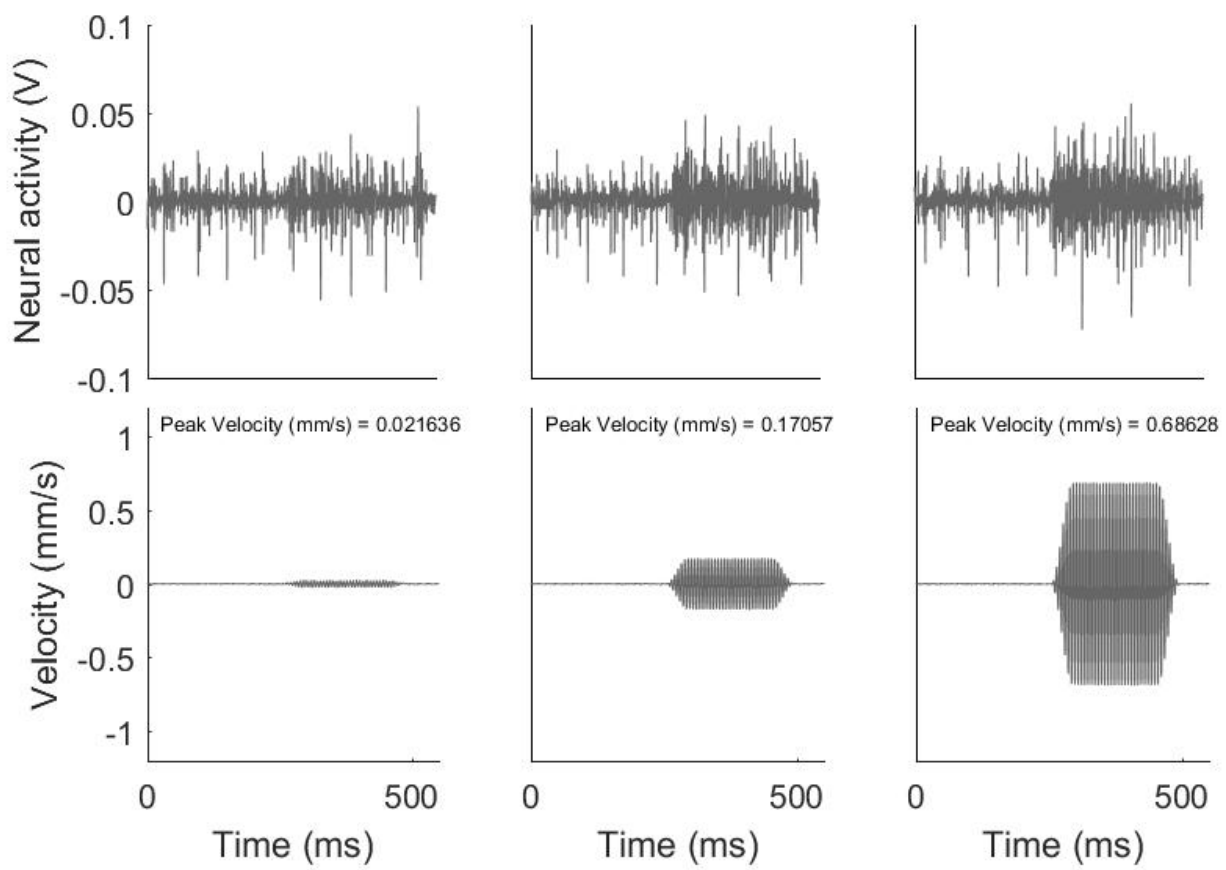

\section{Frequency: $320 \mathrm{~Hz}$}
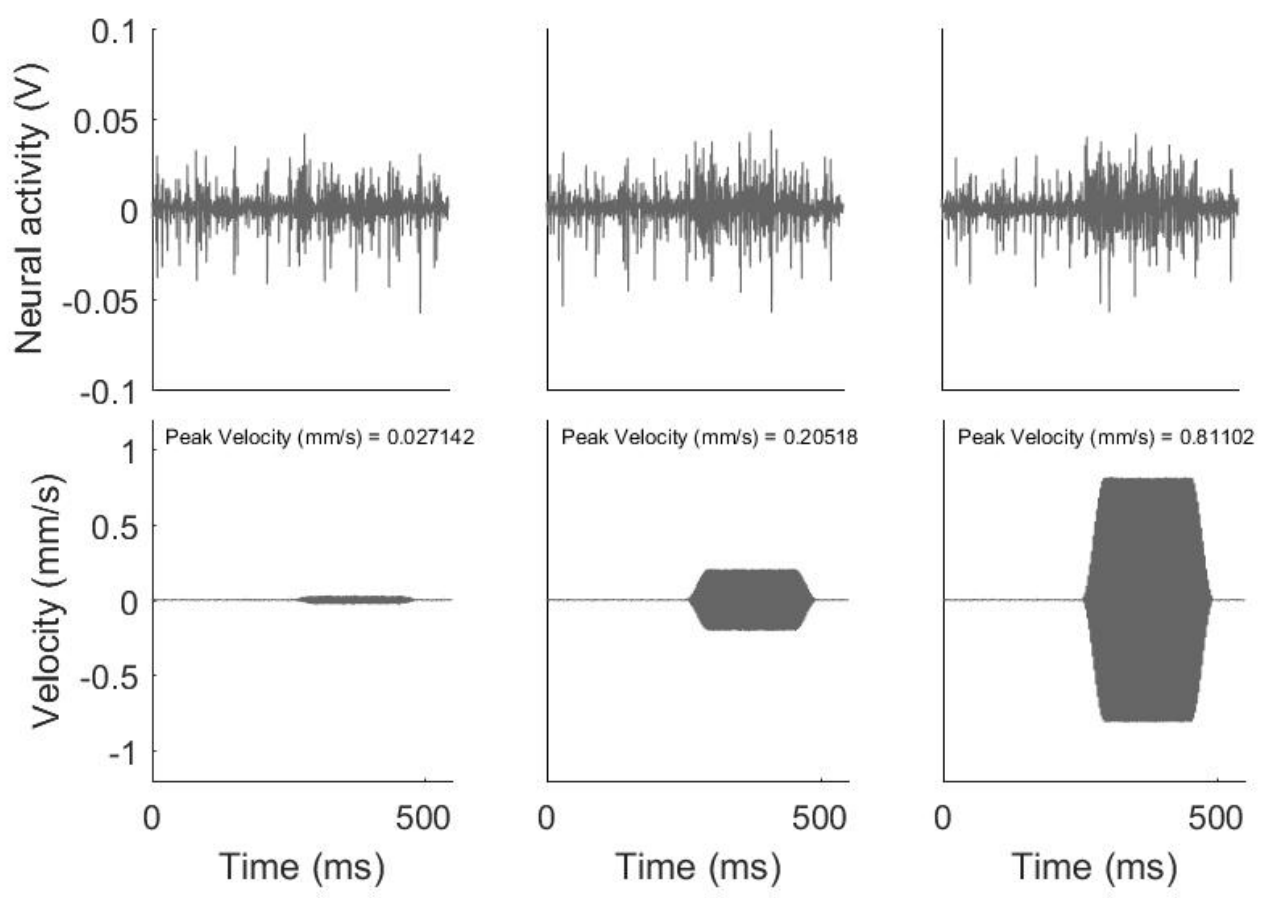


\section{Frequency: $640 \mathrm{~Hz}$}
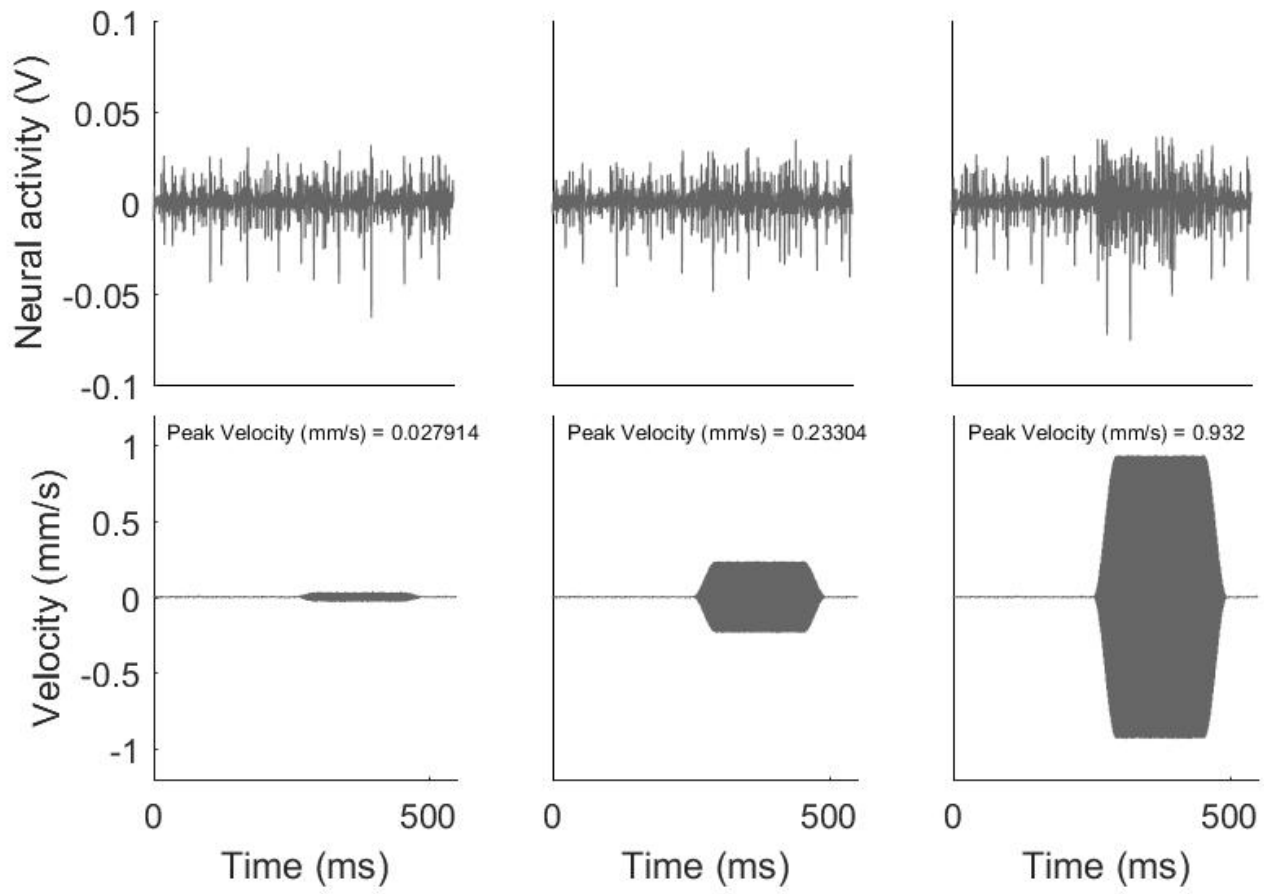
Appendix B.3 - Sample traces of neural activity from a single proleg hair in D. arcuata

Sample traces of neural activity from a single proleg hair in D. arcuata responding to sine stimuli of $10 \mathrm{~Hz}$ and different intensities.

\section{Frequency: $10 \mathrm{~Hz}$}
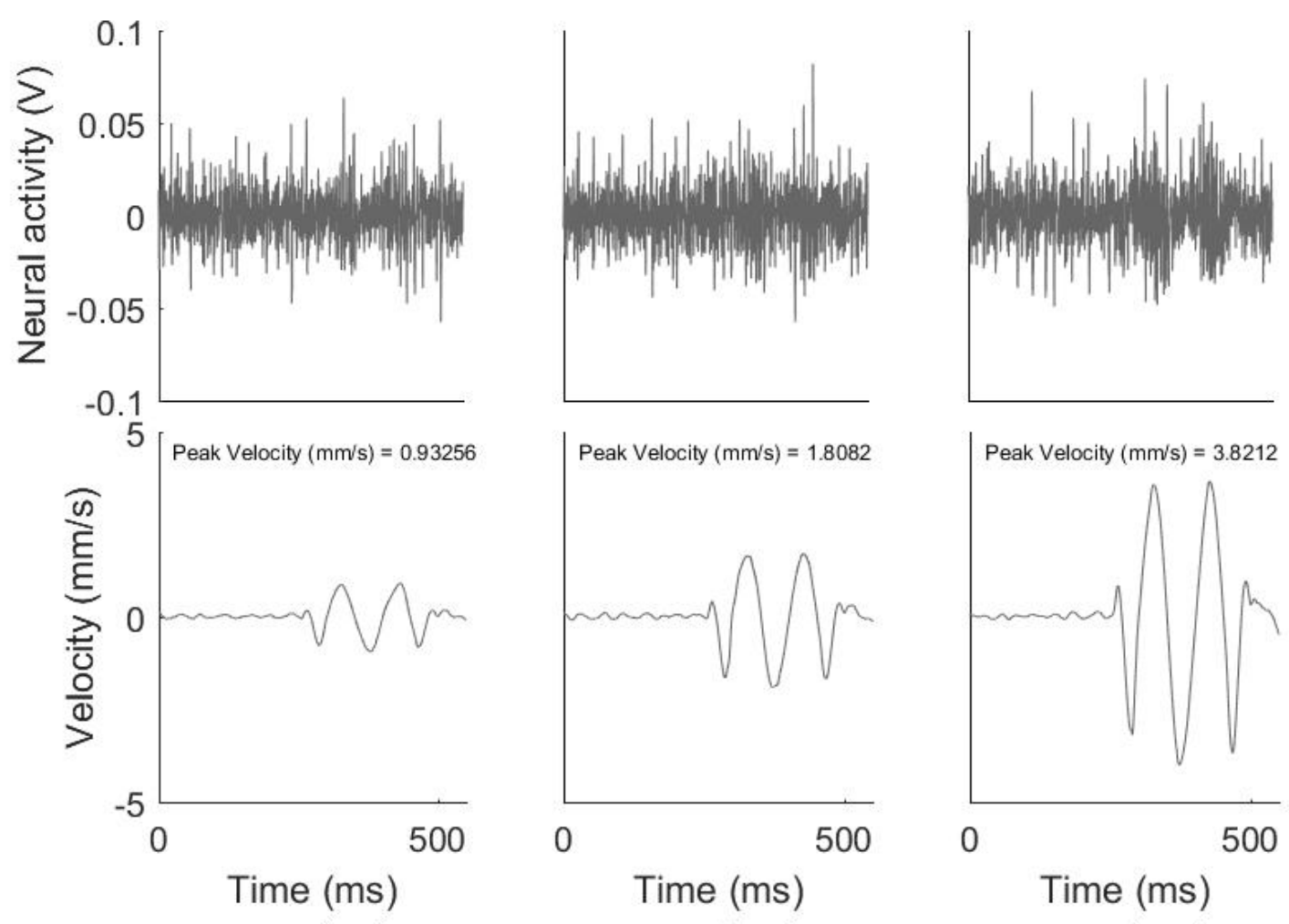


\section{Appendix C (Supplements to Chapter 4)}

Appendix C.1 - Technical details on Micro-CT scanning

This section provides some details about preparation of Drepana arcuata caterpillars for performing Micro-CT scanning. While the first step of this procedure, i.e. fixing, was conducted on Summer 2013 during my exchange program in Carleton University and on Summer 2015 during my visit to participate to the ISV 2015, all the others were conducted later on December 2015, after I had already returned to Brazil. The procedure here described was modified from methods suggested by Eberhard et al. (2010).

\section{FIXATIVES AND STORAGE SOLUTIONS}

I fixed my samples by injecting and immersing them in Bouin's alcoholic. For transportation in the airplane, the samples were transferred to alcohol $70 \%$. This procedure was taken in July 2015.

\section{STAINS}

Before staining, I cut the specimens dividing the thorax from the abdomen to allow a better stain infiltration. At my research I tested two stains:
1) $\operatorname{PTA}(0.3 \%$ phosphotungstic acid $+29.7 \%$ water $+70 \%$ ethanol $)$;
2) I2 (1\% Iodine methalic $+99 \%$ ethanol). 
For my specimens, I2 was by far the best stain. The chemical was easier to find and simpler to use. The PTA had many limitations. For example, it had very low permeability through the tissues. I left my samples immersed in it for a long time (48hs), and the images still ended up having a very low contrast. The cuticle was not stained at all and was invisible at the final images of the micro-CT. The iodine on the other hand was very efficient. Two of the samples that I tried staining first with PTA (which did not

work) I tried staining afterwards with Iodine (which worked very well). Less than 1 hour in the $1 \%$ iodine solution was enough for giving the samples the contrast that I obtained in the models.

\section{STAINING WITH I2}

After having the samples fixed and stored in alcohol $70 \%$, I serially dehydrated them in alcohol solutions until up to $95 \%$. Then I transferred the samples to the Iodine solution, where they stayed for about 40 minutes (Eberhard et al., 2010 suggests overnight though). I washed the samples with alcohol $95 \%$ and scanned them inside a microcentrifuge tube, also filled with alcohol $95 \%$.

\section{SCANNER AND SOFTWARE}

The facility where I scanned my samples was the Brazilian Nanotechnology National Lab (LNNano) in the Brazilian Center for Research in Energy and Materials (CNPEM). They promptly welcome international researchers who are seeking to use their facilities with no charge, including the Micro-CT. (http://lnnano.cnpem.br/laboratories/lmn/facilities/x-raymicrotomograph/). The equipment used was an X-ray Microtomograph Skyscan 1272 (Bruker, Billerica, USA). The lab technician set it up for scanning my samples. The 
output data consisted of reconstructed slice-by-slice grey-scale radiographs of the samples, which could be loaded in CTVox software to create 3D volume-rendered models (CTVox, Bruker, Billerica, USA).

In this software, artificial colors could be added to the volume-rendered models based on the grey scale of their voxels. Also in this software, short animations were made using two imaging modes: volume, which keeps the grey-scale based colors on the voxels, and Maximum Intensity Projection (MIP), which allows a transparent-like visualisation of internal structures. Animations were saved in video files and used for obtaining images. The Micro-CT image shown in Figure 3.2 came from a MIP model visualization with a voxel size of $1.12 \mu \mathrm{m}$.

Alternatively, the reconstructed slice-by-slice radiographs can be imported into a CTanalyser software (CTan, Bruker, Billerica, USA) for producing binary 3D models. This is done using the thresholding tool, which binarize voxels in either black or white based on their value on the grey scale. This program can be also used to take measurements such as length, area, volume and density. The binarized 3D models can be used to construct surface-rendering images or exported in files for further visualization or analysis. Independently created 3D binary models can be opened, combined and visualized using a third software (CTVol, Bruker, Billerica, USA). Using these software, I generated, measured and visualized 3D binary models, but this was done primary for testing purposes and hence the results are not shown in this thesis. 


\section{Appendix D (Supplementary videos)}

The following videos are part of the accompanying materials in my thesis, but they were too large to be submitted online. Hence, they can be found in the hard drive of my thesis and will be available for an undefined time in the playlist of my YouTube Channel, which is accessible through the link:

"https:/www.youtube.com/playlist?list=PLkrBhw1zGi-xZNT5ZLjI5yjVetYgOT3mO" 


\section{Appendix D.1 - Short tutorial for exposing a caterpillar`s nervous ganglion}

Duration: 03:55 min.

\section{CAPTION}

The steps regarding the dissection process in the video are listed below:

3 - Anesthetize (00:08)

4 - Tie the mid body up (00:17)

5 - Cut thorax off $(00: 25)$

6 - Clean fluids up (00:40)

7 - Pin abdomen down (00:53)

8 - Shorten non-targeted sensory hairs $(01: 14)$

9 - Step 9 is optional and hence is shown in another video (Appendix D.4).

10 - Make superficial incision ventrally to the ganglion (01:27)

11 - Clean way up to ganglion (01:36)

12 - Identify one of the ventral nerves - each innervates a proleg (02:07)

Obs.: At this moment the specimen was rotated to facilitate manipulation of the electrodes.

13 - Separate nerve from trachea using electrodes. The trachea may be cut (02:54)

14 - Hook nerve with Recording electrode (03:17) 
This video shows the dissection of a Drepana arcuata caterpillar and hooking of the ventral nerve with a steel electrode.

After dissection, a neural recording was also conducted to demonstrate that the caterpillar`s ventral nerve was still active. The video demonstrating the neural response can be found in another video (Appendix D.2).

This video is a short edited version of a longer dissection process - about 40 minutes long. The whole procedure was taken and recorded on October 22nd, 2017.

Caterpillar ID: Drepana arcuata \# SS.

Video Capture Device: Main frame - Camera attached to the microscope (DMC4500, Leica Microsystems GmbH); Secondary frame - Camera positioned laterally on a tripod (HDR-HC7, Sony, Tokyo, Japan). 


\title{
Appendix D.2 - Neural activity recorded from ventral nerve
}

Duration: $55 \mathrm{~s}$.

\begin{abstract}
CAPTION
This video is a continuation of the dissecting tutorial found in another video (Appendix D.1).

In this video, the computer screen shows the ventral nerve of one abdominal ganglion in a Drepana arcuata caterpillar hooked by a steel electrode. A reference electrode is also shown. The ventral nerve is visibly de-efferented, i.e. the portion hooked by the electrode has no connection with the ganglion (central nervous system) while the end that innervates the proleg is intact.
\end{abstract}

When the nerve is raised from saline, the oscilloscope shows voltage variations (spikes) due to recorded action potentials. Simultaneously, sounds representing these spikes can be heard from the audio monitor. Since the nerve is de-efferented, the spikes were likely originated in sensory neurons.

Caterpillar ID: Drepana arcuata \# SS.

Video Capture Device: Mobile phone camera filmed the experimental set-up (SMJ120W, Samsung, Seul, South Korea) while camera attached to the microscope filmed the specimen and displayed the image on the PC screen (5MP Digital Image, Celestron, Torrance, USA). 


\section{Appendix D.3 - What might happen when the caterpillar wiggles}

Duration: 27 s.

\section{CAPTION}

This video shows a specimen that was ready to be recorded from, but it moved and the gut inflated. It took me 30 minutes to deflate the gut and clean everything before resuming recordings.

Recordings were still successful after this.

The procedure for deflating the gut is shown in another video (Appendix D.4).

Caterpillar ID: Trichoplusia $n i \# 18$.

Video Capture Device: Camera attached to the microscope (5MP Digital Image, Celestron, Torrance, USA). 


\section{Appendix D.4 - How to do deflate the gut}

Duration: $47 \mathrm{~s}$.

\section{CAPTION}

This video corresponds to step \#9 of my dissecting protocol for exposing the nervous ganglion of a caterpillar.

In some cases, the caterpillar contracts and the gut becomes pressurized, inflating through the access hole, dragging the nervous ganglion and making it difficult to hook its nerves.

Step \#9 shows how to solve this situation while reducing damage to the nervous system.

The other steps of the tutorial are shown in the video in another video (Appendix D.1).

See what might happen if the gut is not deflated in advance in another video (Appendix D.3).

Caterpillar ID: Drepana arcuata \# SS.

Video Capture Device: Camera attached to the microscope (DMC4500, Leica Microsystems GmbH) 


\section{Appendix D.5 - Gripping hair at antero-posterior direction}

Duration: $17 \mathrm{~s}$.

\section{CAPTION}

This video shows the exact moment in which I used the forceps to grip the caterpillar`s proleg hair at antero-posterior direction.

A video showing the forceps gripping the hair at antero-posterior direction is shown in another video (Appendix D.6).

This caterpillar was submitted to sine and step stimuli in both latero-ventral and antero-posterior direction.

Caterpillar ID: Trichoplusia ni \#18.

Video Capture Device: Camera attached to THE microscope (5MP Digital Image, Celestron, Torrance, USA). 


\section{Appendix D.6 - Gripping hair at latero-medial direction}

Duration: 32 s.

\section{CAPTION}

This video shows the exact moment in which I used the forceps to grip the caterpillar`s proleg hair at latero-medial direction.

A video showing the forceps gripping the hair at antero-posterior direction is shown in another video (Appendix D.5).

This caterpillar was submitted to sine and step stimuli in both latero-ventral and antero-posterior direction.

Caterpillar ID: Trichoplusia ni \#18.

Video Capture Device: Camera attached to the microscope (5MP Digital Image, Celestron, Torrance, USA). 


\title{
Appendix D.7 - $10 \mathrm{~Hz}$ sine vibration
}

Duration: $14 \mathrm{~s}$.

\begin{abstract}
CAPTION
This video shows a series of sine stimuli. All of them have frequency of $10 \mathrm{~Hz}$, total duration of $250 \mathrm{~ms}$, peak-velocity of $1.43 \mathrm{~mm} / \mathrm{s}$ peak-velocity and peak-to-peak displacement of $46.2 \mu \mathrm{m}$.

The stimuli are being applied in the latero-ventral direction.

The sounds heard at this video when the forceps vibrate represent the neural activity recorded from the nerve. They and are being played by the speakers of an audio monitor in the room. Since the ventral nerve was de-efferented, the response is likely sensory, not motor.
\end{abstract}

Step vibrational stimuli can be seen in another video (Appendix D.9).

Caterpillar ID: Trichoplusia ni \#7.

Video Capture Device: Mobile phone camera carefully held on top of the microscope`s objective lens (SM-J120W, Samsung, Seul, South Korea). 


\section{Appendix D.8 - $80 \mathrm{~Hz}$ sine vibration}

Duration: $23 \mathrm{~s}$.

\section{CAPTION}

This video shows a series of sine stimuli. All of them have frequency of $80 \mathrm{~Hz}$, total duration of $250 \mathrm{~ms}$, peak-velocity of $11.5 \mathrm{~mm} / \mathrm{s}$ peak-velocity and peak-to-peak displacement of $46.0 \mu \mathrm{m}$.

The stimuli is being applied in the latero-ventral direction.

The sounds heard at this video when the forceps vibrate represent the neural activity recorded from the nerve. They and are being played by the speakers of an audio monitor in the room. Since the ventral nerve was de-efferented, the response is likely sensory, not motor.

Step vibrational stimuli can be seen in another video (Appendix D.9).

Caterpillar ID: Trichoplusia ni \#7.

Video Capture Device: Mobile phone camera carefully held on top of the microscope`s objective lens (SM-J120W, Samsung, Seul, South Korea). 


\title{
Appendix D.9 - Step stimulus (single pull/push)
}

Duration: $12 \mathrm{~s}$.

\begin{abstract}
CAPTION
The video shows a series of step stimuli that increase in amplitude, reaching a maximum amplitude (00:09) of $0.79 \mathrm{~mm} / \mathrm{s}$ peak-velocity and $18.1 \mu \mathrm{m}$ peak-to-zero displacement.

In this video, it can be observed that the forceps pull and then push the hair very quickly. Here, each pull is a stimulus applied in the lateral direction while each push is applied in the ventral direction.

Sine vibrational stimuli can be seen in other two videos (Appendix D.7 and Appendix D.8).

Caterpillar ID: Trichoplusia ni \#18

Video Capture Device: Camera attached to the microscope (5MP Digital Image, Celestron, Torrance, USA).
\end{abstract}

